\title{
THESIS ON
}

\section{CONTROL OF BUCK CONVERTER BY POLYNOMIAL, PID AND PD CONTROLLERS.}

\author{
BY \\ ERCS. Madhu Kiran 850923-1232 \\ $\boldsymbol{\&}$ \\ THOTA. Partha Saradhi 850715-6175
}

This Thesis is presented as a part of our Degree in Master of Science in Electrical Engineering.

Blekinge Institute of Technology [BTH]

School of Engineering Supervisor: Anders Hultgren

$$
\text { June - } 2012
$$


Abstract

Contents:

Names:

Page no:

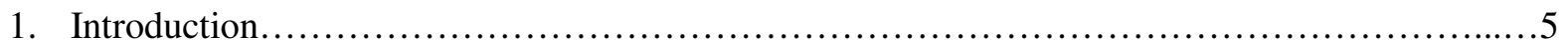

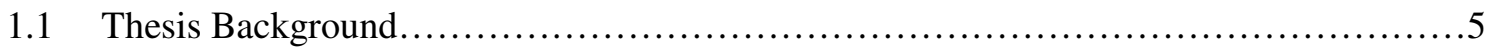

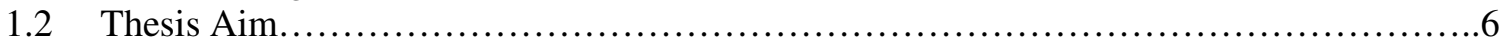

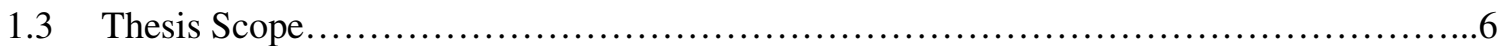

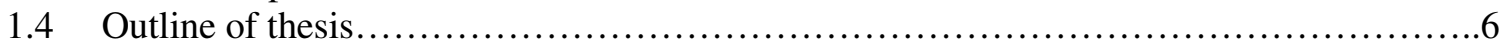

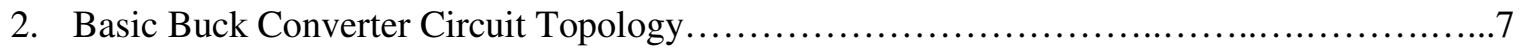

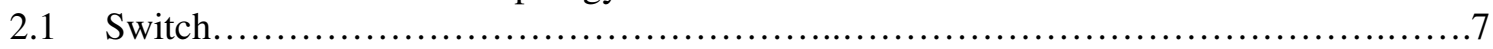

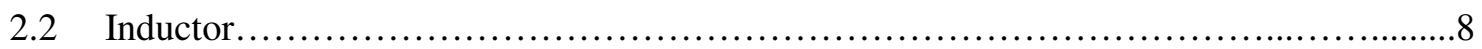

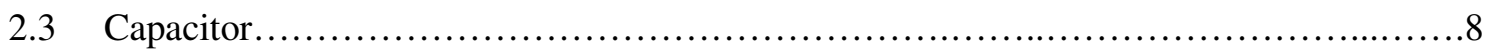

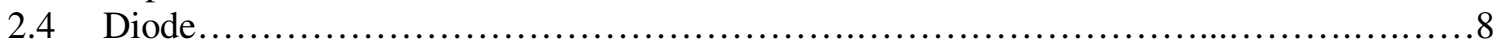

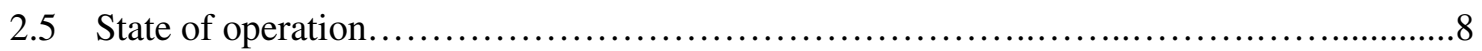

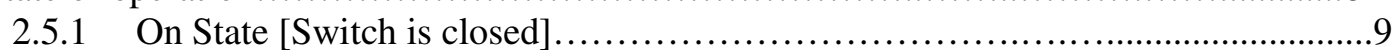

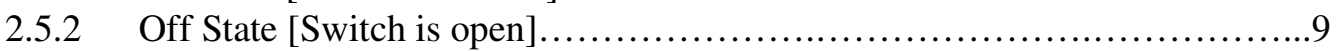

2.5.3 Modes of Operation....................................................... 10

2.5.4 Ericsson BMR 450 Feature................................................ 10

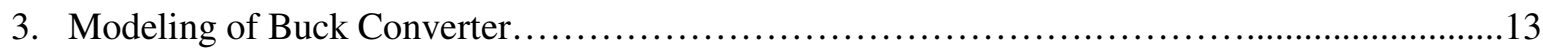

3.1 Mathematical equations of the buck converter when the switch is in ON state............13

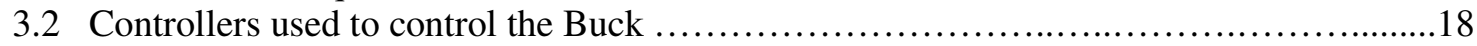

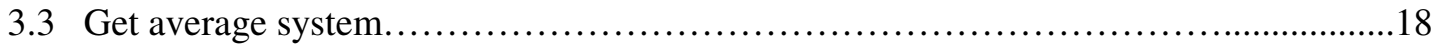

4. Polynomial Pole Placement Controller.................................................

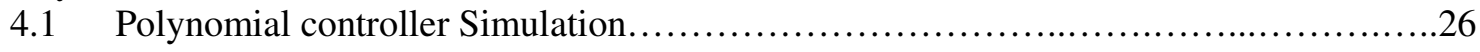

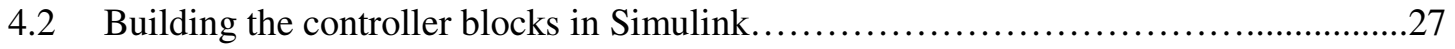

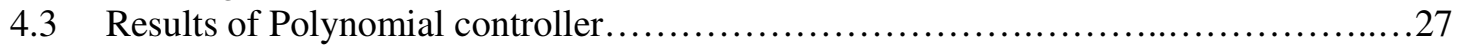

4.3.1 Simulation results of the Continuous and discrete model ........................28

4.3.2 Simulation result without disturbance and without noise.....................30

4.3.3 Simulation result without disturbance and with noise........................31

4.3.4 Simulation results with disturbance and without noise.......................33

4.3.5 Simulation results with disturbance and with noise...........................35

4.3.6 Simulation results with large load capacitor............................... 35

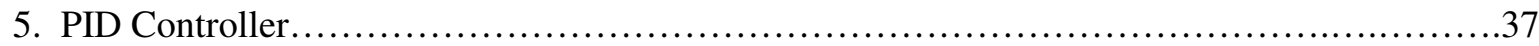

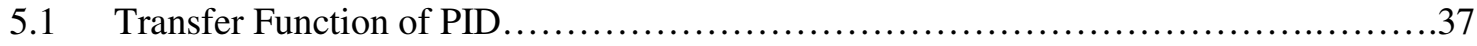

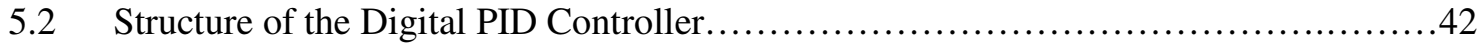

5.3 Simulation results of the Continuous and discrete model............................44

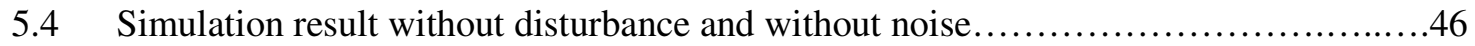

5.5 Simulation result without disturbance and with noise ............................. 47

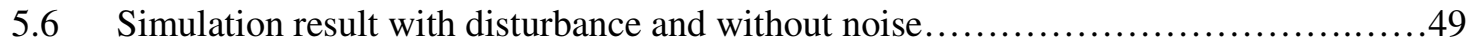

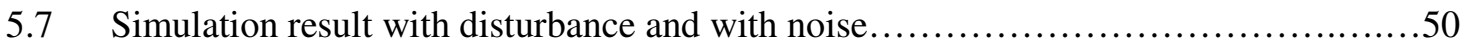




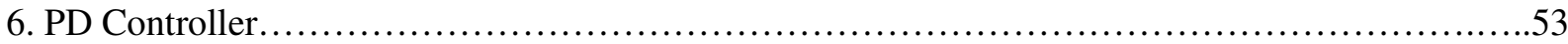

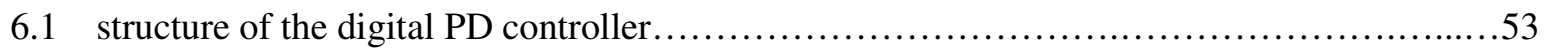

6.2 calculation for finding the poles of PD controller of second order.....................55

6.3 simulation results of the continuous and discrete model of PD controller..................58

6.4 simulation results without disturbance and with noise...............................61

6.5 simulation results with disturbance and without noise...............................61

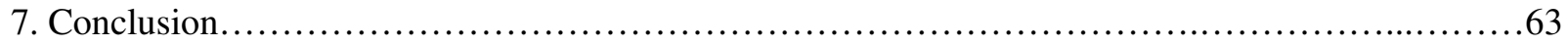

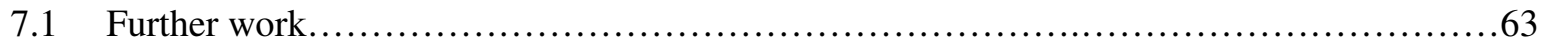

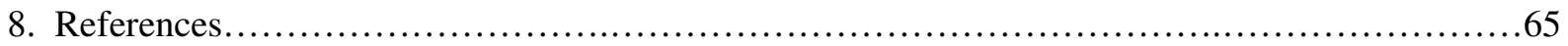

\section{Appendix}

Appendix 1: Mathematical equation of buck converter when switch is ON..................67

Appendix 2: Solving the equation for obtaining the Polynomials ...................... 73

Appendix 3: Resulted graphs for determining the pole for Polynomial controller ...........75

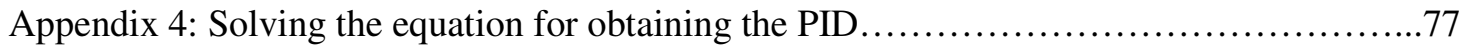

Appendix 5: Resulted graphs for determining the pole for PID controller...................79

Appendix 6: Solving the euation for obtaining the PD Controller.........................81

Appendix 7: Resulted graphs for determining the pole for PD controller..................83

Appendix 8: Matlab code for the Polynomial Pole Placement Controller....................87

Appendix 9: Matlab code for PID Controller........................................ 91

Appendix 10: Matlab code for PD Controller......................................95 


\section{Abstract:}

This thesis is an ongoing project of Ericsson with collaboration of Blekinge Institute of Technology [BTH], and Linneaus University [LNU] to compare the functionality and performance of three controllers Polynomial Pole Placement, PID [Proportional Integral Derivative] and PD controller in third order.

This paper presents the state space modeling approach of DC-DC Buck converter. The main aim of this thesis is, by considering the buck converter system of Ericsson BMR450 with the PID, POLYNOMIAL and PD controllers at feedback loop, thus running their Matlab file with their appropiate Simulink block diagram, and comparing the three controllers performance by verifying their appropiate output graphs.

The third order controller design is complicated and response is slow. The second order design is easy and gives better responses than third order Polynomial, PID and PD controllers.

\section{Index Terms:}

Buck Converters, DC-DC Converters, PID, PD \& POLYNOMIAL Controllers. 


\section{Chapter 1. Introduction:}

Over the past three to four decades, the conventional use of electrical components is growing very rapidly. So, the competition is inevitable in making the devices as small as possible to satisfy customer needs. In which, the power consumption is one of the important factor for every electrical component to run efficiently.

\subsection{Thesis Background:}

As the electrical components are packed together, the power conversion mechanisms are changed as linear regulators, which can provide very high quality output voltage and for higher power levels, switching regulators were used. Switching regulators use power electronic semiconductor switches in ON and OFF states, because there is a small power loss in those states [low voltage across a switch in the on state, zero current through a switch in the off state], thus switching regulators can achieve high efficiency energy conversion. The switchedmode power supply [SMPS] is a prominent element in processing the electrical power, in power electronics field [1].

A Buck converter is a DC-DC voltage step down converter. Because of its high efficiency over a wide range of load current, this device is one of the extensively used DC-DC converter topologies in power management systems.

For these requirements, the Buck converters are used for fast load line transient response and also for high efficiency over a large load current range [2]. For example, in a computer motherboard the load voltage can be regulated to $5 \mathrm{v}$ or $3.3 \mathrm{v}$ to satisfy the requirements of Integrated circuits [IC] or sub-circuits. Here the voltage is constant unlike the batteries, where the voltage declines after a certain period of operation.

By using the same circuit with use of switched modes different voltage levels can be obtained [3]. The switching will be controlled by Pulse Width Modulator [PWM] signals. It constantly maintains voltage range regardless of the load current variation and the voltage drop of the battery supply [4]. 
The non-isolated converters were usually employing an inductor, with no dc voltage isolation between the input and the output. In most of the converters usage, dc isolation is not necessary.

\subsection{Thesis Aim:}

The main objective of this thesis is to design a buck converter controller based on the theory for discrete Polynomial PD and PID Controller and also evaluating their performances, For the evaluated polynomial PD and PID controller can be found in [5], and [6]. The control design should be evaluated by means of accuracy in output voltage level given some different kind of disturbances which are, load current changes, measurement noise and parameter variations.

\subsection{Thesis Scopes:}

The following are scopes of this thesis:

- Study the operation of Buck Converter.

- Design the mathematical system model.

- Design the Polynomial PD and PID Controller.

- Simulation of Buck Converter and Controller circuit using SIMULINK and in MATLAB, for testing the properties of the system.

- Final conclusion from the simulation result.

- Reference and Appendix.

\subsection{Outline of Thesis:}

There are 6 chapters, present in this thesis, here First chapter tells about the thesis objective, introduction and scope. The Second chapter explains about the background of the Buck Converter circuit. The mathematical system models of the Buck Converter were built in Third chapter. The Polynomial Pole Placement and PID Controllers design with simulations implemented on Matlab and Simulink were presented in Fourth and Fifth chapters. The Chapter sixth tells about the results and conclusion obtained with future work, and the final part provides the references and appendix used in this thesis work. 


\section{Chapter 2. Basic Buck Converter Circuit Topology:}

The basic buck converter operates in two modes i.e., switch ON and switch OFF states. Here these two modes are briefly explained with the help of below Figure 1.

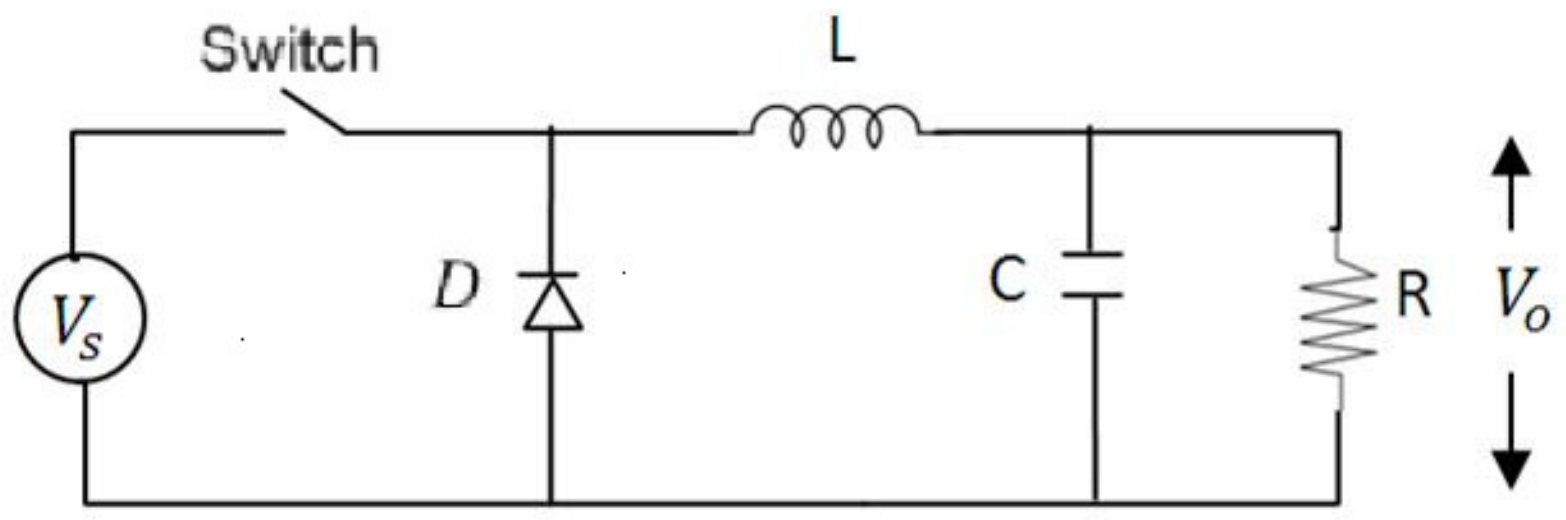

Figure 1: Basic Buck Converter with a resistive load.

Where $\mathrm{V}_{\mathrm{S}}=$ voltage source, $\mathrm{D}=$ diode, $\mathrm{L}=$ inductor, $\mathrm{C}=$ capacitor, $\mathrm{R}=$ resistor and $\mathrm{V}_{0}=$ load voltage, When the switch is closed, the input voltage is connected to the inductor and the load voltage. Thus the energy will be stored as the current increases in the inductor. And the capacitor begins to discharge its voltage. When the switch is open, the inductor discharges its energy to the load resistor, thus decreasing in current will occur in the system gradually.

In this case, the inductor acts as a source to the load to keep the flow of current without interruption in the circuit. The functions of elements in the above Basic Buck Converter are explained below.

\subsection{Switch:}

The switch is basically a power switch or a transistor. At the gate of the transistor, the PWM signal is introduced to control the ON and OFF state of the transistor. During the switch ON, the supply voltage is equal to the sum of the voltages across the inductor and resistor at load. During the switch OFF, the voltage across the inductor is equal to voltage across the resistor at load. By varying the PWM signal between the switch ON and OFF states we can control the average output voltage. 


\subsection{Inductor:}

The inductor main function is to supply power constantly to the load resistor. In OFF state no power is supplied by the voltage source, and thus the inductor acts as a source at this stage by transferring the current to the load as shown in Figure 2.

\subsection{Capacitor:}

The capacitor reduces the ripple in the output voltage and here the capacitor wave-off output voltage by filtering away the harmonic currents away from the load. Capacitance across the load is very important for reducing the voltage and ripples present at the output of a step-down converter.

\subsection{Diode:}

When the switch is open [OFF], a current path should be present for the inductor current, this path is provided by the catch diode [freewheeling diode]. The purpose of this diode is not to rectify, but to direct current flow in the circuit and to ensure that there is always a path for the current to flow into the inductor. It is also necessary that this diode should be able to turn off relatively fast. Thus the diode enables the converter to convert stored energy in the inductor to the load.

When the switch closes, the current rises linearly [exponentially if resistance is also present].When the switch opens, the freewheeling diode causes a linear decrease in current. At steady state we have a saw tooth response with an average value of the current.

To maintain a constant output voltage in normal operation conditions, the feedback loop and control circuitry are carefully nested around the desired circuits and control by pulse-width modulation is necessary for regulating the output.

\subsection{States of Operation:}

The operation of the DC-DC converter is simple, it works in two states i.e., ON and OFF states. The explanation of $\mathrm{ON}$ state is as follows. 


\subsubsection{ON State (Switch is closed):}

During the ON state, the closed switch results in transferring the energy from the source voltage [Vs] to the inductor [L], thus the diode becomes reverse bias. In this state, current through the inductor increases gradually, as shown in Figure 2.

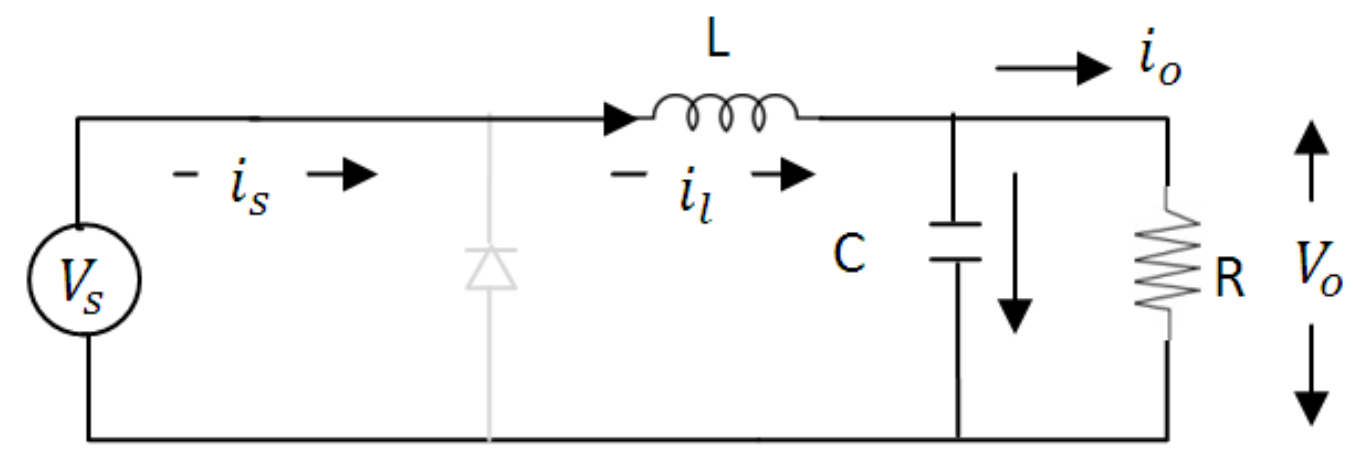

Figure 2: Buck Converter in ON state.

\subsubsection{OFF State (Switch is open):}

During the OFF state, the switch is open, now the inductor acts as a source by maintaining constant energy transfer to the resistor at load. At this stage, the diode starts conducting and the current through the circuit decreases linearly as the energy in the inductor discharges as shown in Figure 3.

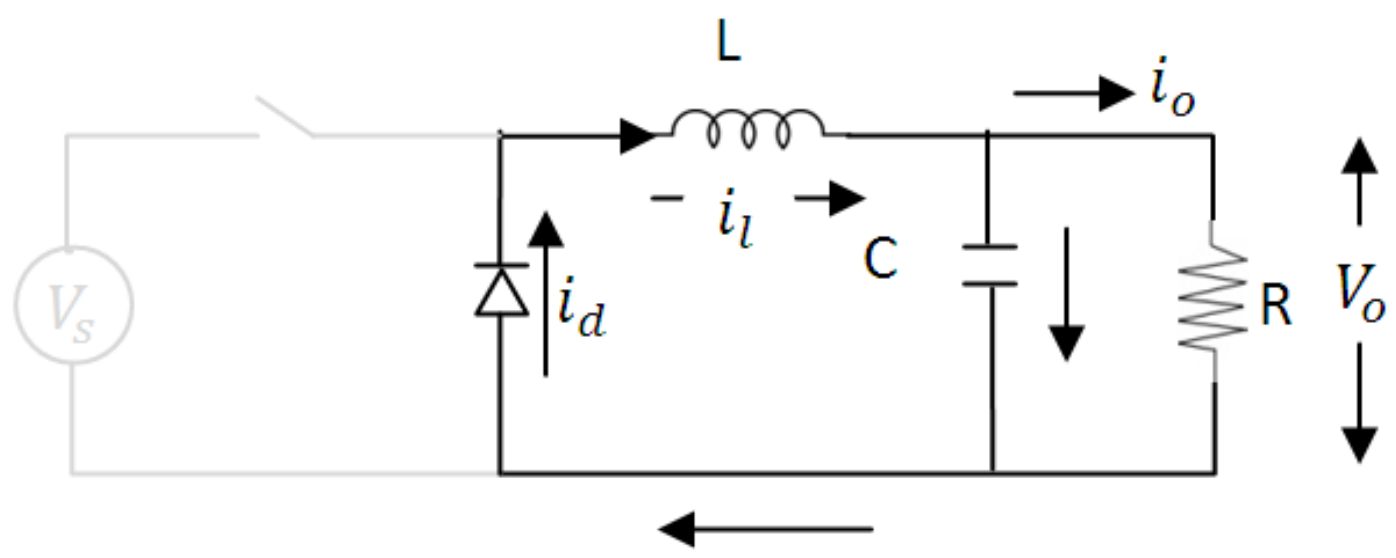

Figure 3: Buck Converter in OFF state.

The Figure 4 graph is obtained when the switch is in ON and OFF states and the response of the current across the diode and load, where shown accordingly. 

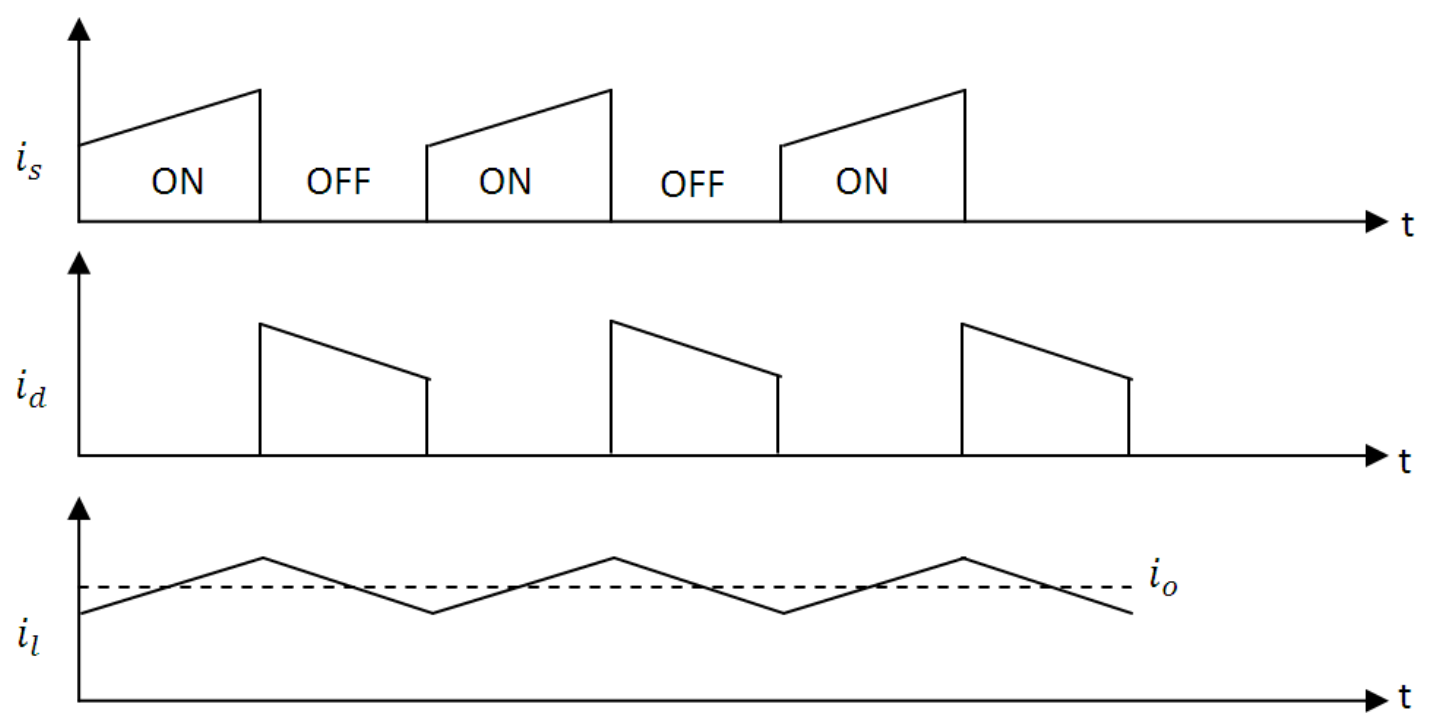

Figure 4: Diode and the load during the two switch states of operation of buck converter, where $\mathrm{i}_{0}$ represents the average current across the load.

\subsubsection{Modes of operation:}

The buck converter operates in two modes i.e., continuous and discontinuous modes of operation. In continuous mode, the current passing through the inductor doesn't falls to zero.

But in discontinuous mode, the current passing through the inductor reduces to zero during its part of the switching period. This phenomenon exists due to the time and energy required by the load is enough when compared to the complete operating period. In this thesis, we used continuous mode of operation.

\subsubsection{Ericsson BMR 450 Features:}

This BMR450 is one of the first products developed by Ericsson in digital power [computerizing the DC-DC converters] the other models are BMR 451 and 453, presently it is the main focus of the DC-DC converter area today. 


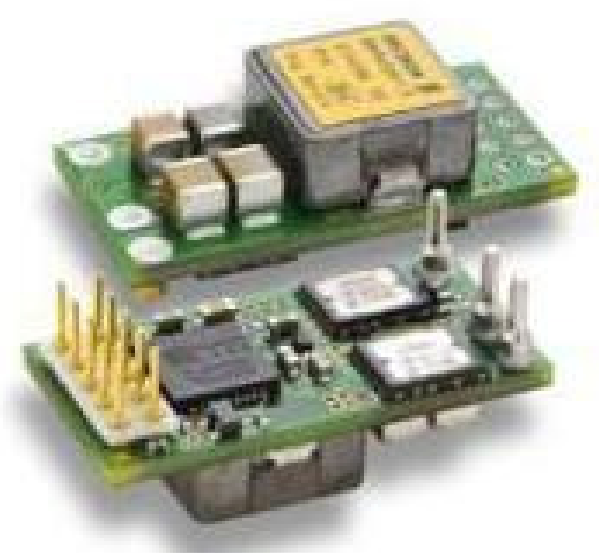

Figure 5: Ericsson BMR 450

Digital PWM with adaptive dead-time control, precision delay and ramp-up are the main features of BMR450. Other features are listed below.

Input: 4.5-14 V, Output: $20 \mathrm{~A} / 100 \mathrm{~W}$.

- Small package $25.65 \times 12.9 \times 8.2 \mathrm{~mm}$ [1.01 x $0.51 \times 0.323 \mathrm{in}]$.

- 20 A output current.

- $4.5 \mathrm{~V}$ - $14 \mathrm{~V}$ input voltage range.

- $0.6 \mathrm{~V}-5.5 \mathrm{~V}$ output voltage range.

- High efficiency, typ. $96.8 \%$ at half load, $5 \mathrm{~V}_{\text {in }}, 3.3 \mathrm{~V}_{\text {out }}$.

- 5 million hours MTBF.

- Through hole and surface mount versions.

- PM Bus complaint.

- Voltage/current/temperature monitoring.

- Precision delay and ramp-up.

- Non-linear transient response.

- Wide output voltage adjust function.

- Start up into a pre-biased output safe.

- Output short-circuits protection. 
- Over temperature protection.

- ON/OFF inhibit control.

- Output voltage sense.

- ISO 9001/14001 certified supplier. 


\section{Chapter 3. Modeling of Buck Converter:}

The buck converter circuit is transformed to graph using graph theory as shown below.

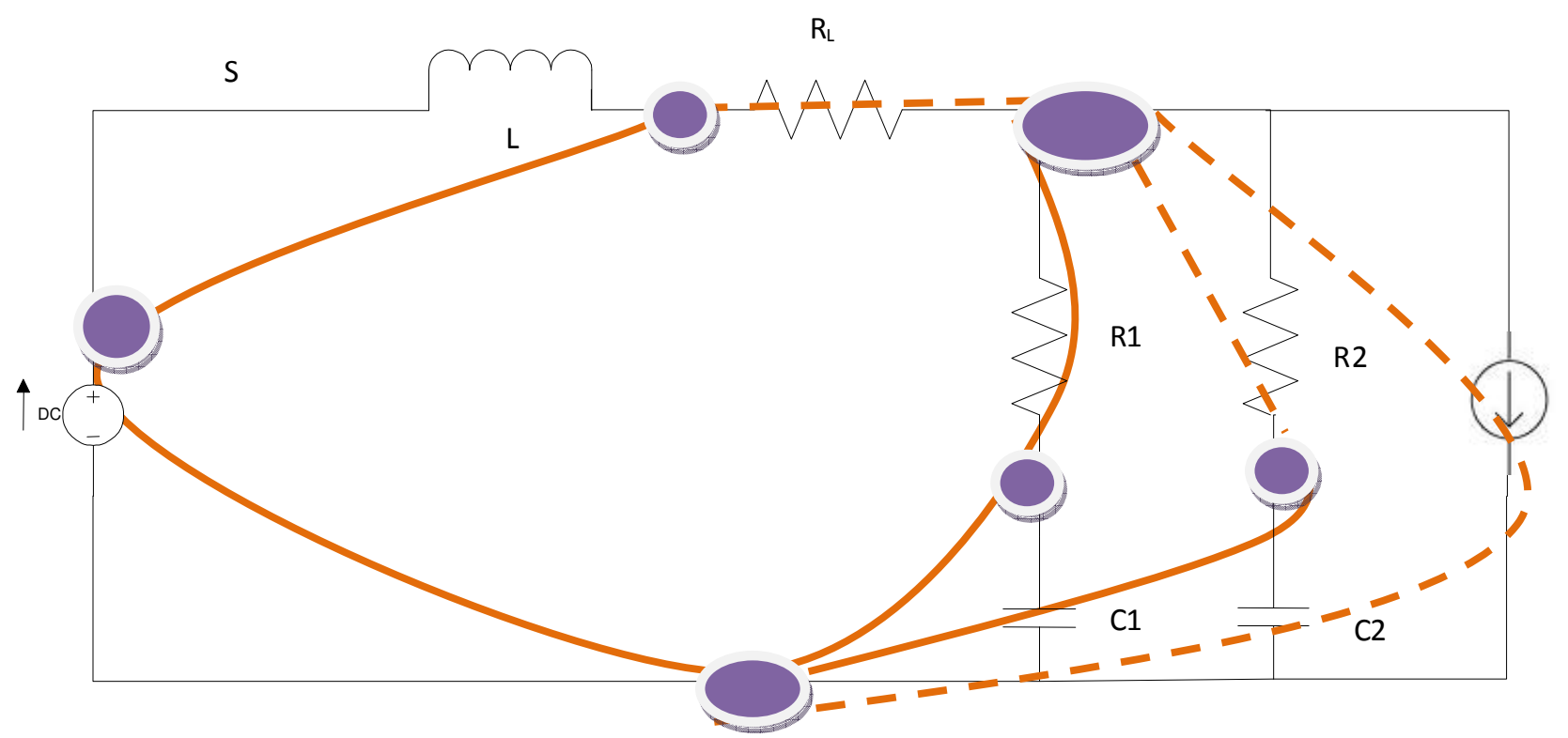

Figure 6: Buck converter model after implementing cut-set method.

\subsection{Mathematical equations of the buck converter when the switch is in ON state:}

To the above Figure 6, Cut-Set method is applied to obtain the tree branch, this is shown in the below Figure 7 , in this figure a cut $\left[i_{u}\right]$ is made across the branch [twig], this $i_{u}$ direction depends up on the direction of the branch. Now this $i_{u}$ cut has the direction, if the link direction [indicated in green color no: 7] is same as the cut direction $\left[i_{u}\right]$ then we can place ' 1 ' under number 7 across $i_{u}$ in the matrix shown below [Matrix 1].

Similarly if we consider [iC1] which cuts through three links indicated with 6,7 and 8 arrows were the link indicated with number 6 and 8 has opposite direction as compare with the cut [iC1], so we had written ' -1 ' under numbers 6 and 8 across iC1, where as the link 7 is in the same direction as that of the twig direction, so ' 1 ' is placed in the below matrix, if the twig does not cut the link then ' 0 ' is placed in the matrix. Like this we had fill the below matrix for all the five cut's i.e., $\left[i_{U}, i_{R L}, i_{R 1}, i_{C 1}, i_{C 2}\right]$. 


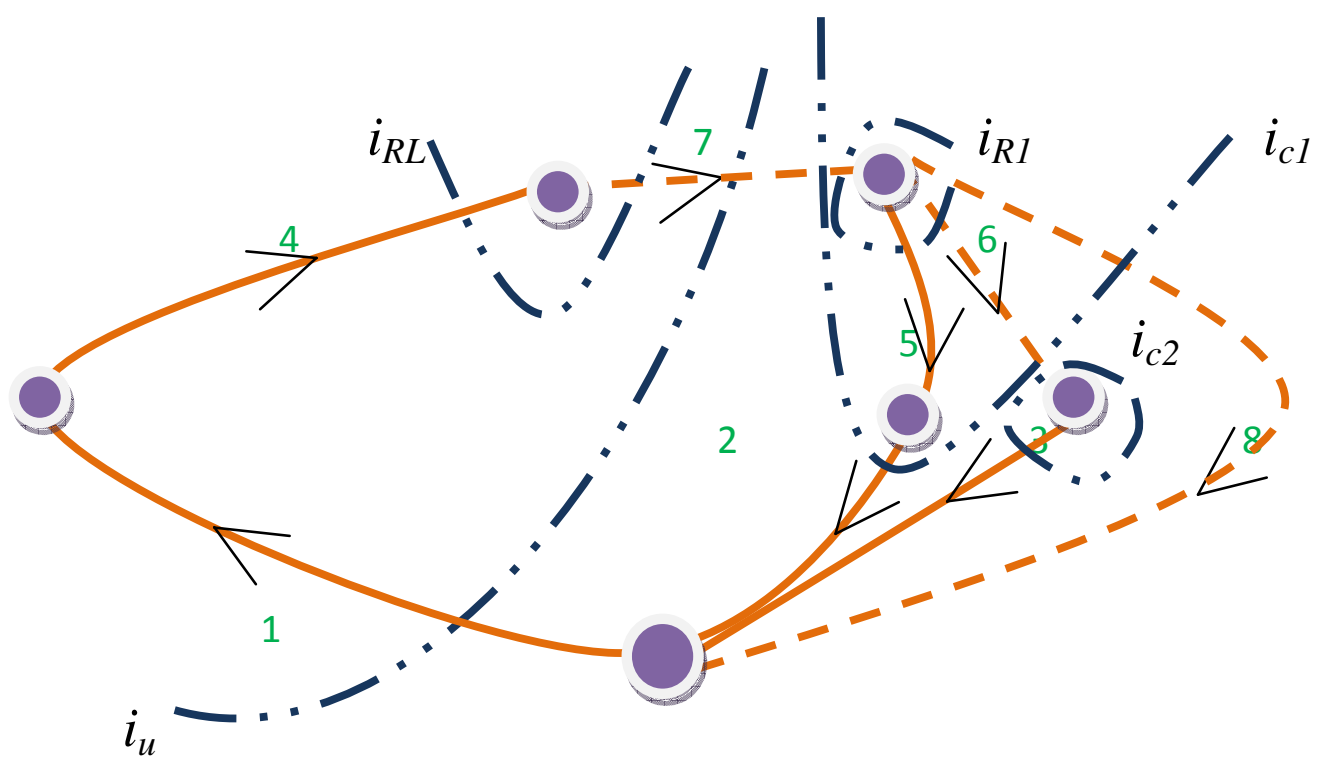

Figure 7: When Cut-Set method is applied to the graph in Figure 6.

$$
\left(\begin{array}{c}
1 \\
i_{U} \\
i_{C 1} \\
i_{C 2} \\
i_{R L} \\
i_{R 1}
\end{array}\right)=\left(\begin{array}{ccccccccc}
0 & 0 & 0 & 0 & 0 & 0 & 1 & 0 \\
0 & 0 & 0 & 0 & 0 & -1 & 1 & -1 \\
0 & 0 & 0 & 0 & 0 & 1 & 0 & 0 \\
0 & 0 & 0 & 0 & 0 & 0 & 1 & 0 \\
0 & 0 & 0 & 0 & 0 & -1 & 1 & -1
\end{array}\right)\left(\begin{array}{c}
u_{U} \\
u_{C 1} \\
u_{C 2} \\
u_{R L} \\
u_{R 1}
\end{array}\right)
$$

Matrix 1: The above equation is the, result of the cut-set branch direction as shown in the Figure 7.

Where, $\mathrm{i}_{\mathrm{U}}=$ current through the voltage source, $\mathrm{i}_{\mathrm{C} 1}=$ current through the capacitor $\mathrm{C} 1$,

$$
\begin{aligned}
& \mathrm{i}_{\mathrm{C} 2}=\text { current through the capacitor } \mathrm{C} 2, \mathrm{i}_{\mathrm{RL}}=\text { current through the resistor } \mathrm{RL} \text {, } \\
& \mathrm{i}_{\mathrm{R} 1}=\text { current through the resistor } \mathrm{R} 1,
\end{aligned}
$$

Now we have to write the matrix for the links [current sources] other than branches, for this we have to consider the KCL [Kirchhoff's Current Law] as shown below, 


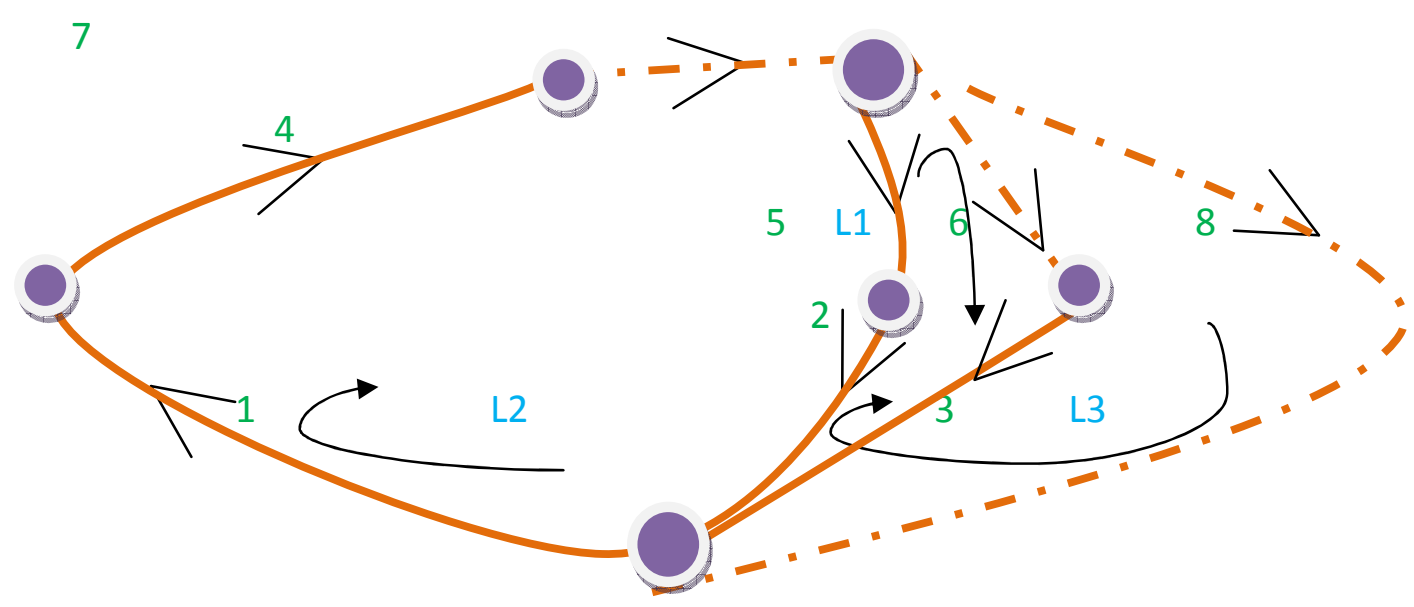

Figure 8: When KCL [Kirchhoff's Current Law] is implemented on Figure 6.

Here the loops are drawn according to the directions of the links, and the below matrix is written depending up on the directions of the loops $[L 1, L 2, L 3]$ with reference to the direction of the links [current source's] i.e., from the above figure 8, loop L1 direction and the branch direction [number 3] are same so we kept ' -1 ', and for branches [number 2 and 5 ] it is ' 1 ' because they are in opposite direction to the loop direction, then for the rest of the branches ' 0 ' is assigned [because loop L1 did not touch the other branches].

And for loop L2, the branches number 1, 2, 4 and 5 are in same direction so we mentioned '- 1 ' and ' 0 ' for the remaining branches as mentioned in the below Matrix 2 is built. Same method for L3 loop also.

$$
\left(\begin{array}{l}
u_{R 2} \\
u_{L} \\
u_{I}
\end{array}\right)=\left(\begin{array}{cccccccc}
0 & 1 & -1 & 0 & 1 & 0 & 0 & 0 \\
-1 & -1 & 0 & -1 & -1 & 0 & 0 & 0 \\
0 & 1 & 0 & 0 & 1 & 0 & 0 & 0
\end{array}\right)\left(\begin{array}{c}
i_{R 2} \\
i_{L} \\
i_{I}
\end{array}\right)
$$

Matrix 2: result of the $\mathrm{KCL}$ links direction as shown in the Figure 8.

Where, $\mathrm{u}_{\mathrm{R} 2}$ in derivative form $\left(R_{2} \frac{d}{d t} i_{R 2}\right)=$ voltage through the resistor $\mathrm{R} 2$,

$\mathrm{u}_{\mathrm{L}}$ in derivative form $\left(L \frac{d}{d t} i_{L}\right)=$ voltage through the inductor $\mathrm{L}$,

$\mathrm{u}_{1}$ in derivative form $\left({ }_{I} \frac{d}{d t} i_{l}\right)=$ voltage through the current source $\mathrm{I}$, 
From the above two Matrices $1 \& 2$, we get the below Matrix 3 [8x8] by combining both the method as shown below,

$$
\left(\begin{array}{c}
i_{U} \\
i_{C 1} \\
i_{C 2} \\
i_{R L} \\
i_{R 1} \\
u_{R 2} \\
u_{L} \\
u_{I}
\end{array}\right)=\left(\begin{array}{cccccccc}
0 & 0 & 0 & 0 & 0 & 0 & 1 & 0 \\
0 & 0 & 0 & 0 & 0 & -1 & 1 & -1 \\
0 & 0 & 0 & 0 & 0 & 1 & 0 & 0 \\
0 & 0 & 0 & 0 & 0 & 0 & 1 & 0 \\
0 & 0 & 0 & 0 & 0 & -1 & 1 & -1 \\
0 & 1 & -1 & 0 & 1 & 0 & 0 & 0 \\
-1 & -1 & 0 & -1 & -1 & 0 & 0 & 0 \\
0 & 1 & 0 & 0 & 1 & 0 & 0 & 0
\end{array}\right)\left(\begin{array}{l}
u_{U} \\
u_{C_{1}} \\
u_{C 2} \\
u_{R L} \\
u_{R 1} \\
i_{R_{2}} \\
i_{L} \\
i_{I}
\end{array}\right)
$$

Matrix 3: final matrix for the graph method shown in the Figure 6.

The above mentioned variables are,

$\mathrm{i}_{\cup}$ in derivative form $\left(u \frac{d}{d t} u_{u}\right)=$ current through the voltage source,

$\mathrm{i}_{\mathrm{C} 1}$ in derivative form $\left({ }_{C 1} \frac{d}{d t} u_{c 1}\right)=$ current through the capacitor $\mathrm{C} 1$,

$\mathrm{i}_{\mathrm{C} 2}$ in derivative form $\left(c_{2} \frac{d}{d t} u_{c 2}\right)=$ current through the capacitor $\mathrm{C} 2$,

$\mathrm{i}_{\mathrm{RL}}$ in derivative form $\left(R L \frac{d}{d t} u_{R L}\right)=$ current through the resistor $\mathrm{RL}$,

$\mathrm{i}_{\mathrm{R} 1}$ in derivative form $\left(R 1 \frac{d}{d t} u_{R 1}\right)=$ current through the resistor $\mathrm{R} 1$,

$\mathrm{U}_{\mathrm{R} 2}$ in derivative form $\left({ }_{R 2} \frac{d}{d t} i_{R 2}\right)=$ voltage through the resistor $\mathrm{R} 2$, 
$\mathrm{u}_{\mathrm{L}}$ in derivative form $\left(L \frac{d}{d t} i_{L}\right)=$ voltage through the inductor $\mathrm{L}$,

$\mathrm{u}_{\mathrm{I}}$ in derivative form $\left({ }_{I} \frac{d}{d t} i_{I}\right)=$ voltage through the current source.

By solving the above shown matrix 3 , we get the $A, B, C$ and $D$ matrix, which are shown below and for the detailed calculation see the Appendix 1.

And changing $u$ into $v$ for the input voltage $[v=-u]$.

$$
\begin{aligned}
& {\left[\begin{array}{c}
i_{C 1} \\
i_{C 2} \\
u_{L}
\end{array}\right]=\left[\begin{array}{ccc}
-1 /\left(R_{1}+R_{2}\right) & 1 /\left(R_{1}+R_{2}\right) & 1-R_{1} /\left(R_{1}+R_{2}\right) \\
1 /\left(R_{1}+R_{2}\right) & -1 /\left(R_{1}+R_{2}\right) & R_{1} /\left(R_{1}+R_{2}\right) \\
-1+R_{1} /\left(R_{1}+R_{2}\right) & -R_{1} /\left(R_{1}+R_{2}\right) & -R_{L}-R 1 R_{1} /\left(R_{1}+R_{2}\right)
\end{array}\right]\left[\begin{array}{c}
u_{C 1} \\
u_{C 2} \\
i_{L}
\end{array}\right]+} \\
& {\left[\begin{array}{cc}
0 & -1+R_{1} /\left(R_{1}+R_{2}\right) \\
0 & -R_{1} /\left(R_{1}+R_{2}\right) \\
1 & R_{1} R_{2} /\left(R_{1}+R_{2}\right)
\end{array}\right]\left[\begin{array}{c}
v \\
i_{I}
\end{array}\right]}
\end{aligned}
$$

And the constitutive equation of the above mentioned matrix equation is,

$$
\begin{aligned}
{\left[\begin{array}{l}
\frac{d}{d t} u_{c 1} \\
\frac{d}{d t} u_{c 2} \\
\frac{d}{d t} i_{L}
\end{array}\right]=} & {\left[\begin{array}{ccc}
-1 /\left(R_{1}+R_{2}\right) C_{1} & 1 /\left(R_{1}+R_{2}\right) C_{1} & 1 / C_{1}-R_{1} /\left(R_{1}+R_{2}\right) C_{1} \\
1 /\left(R_{1}+R_{2}\right) C_{2} & -1 /\left(R_{1}+R_{2}\right) C_{2} & R_{1} /\left(R_{1}+R_{2}\right) C_{2} \\
-1 / L+R_{1} /\left(R_{1}+R_{2}\right) L & -R_{1} /\left(R_{1}+R_{2}\right) L & -R_{L}-R_{1} R_{1} /\left(R_{1}+R_{2}\right) L
\end{array}\right]+} \\
& {\left[\begin{array}{cc}
0 & -1 / C_{1}+R_{1} /\left(R_{1}+R_{2}\right) C_{1} \\
0 & -R_{1} /\left(R_{1}+R_{2}\right) C_{2} \\
1 / L & R_{1} R_{2} /\left(R_{1}+R_{2}\right) L
\end{array}\right] }
\end{aligned}
$$

And for finding the $C$ and $D$ matrices, from the below equation,

$$
\begin{aligned}
& {\left[\frac{d}{d t} i_{R 1}\right]=[1-R 1 /(R 1+R 2) R 1 \quad R 1 /(R 1+R 2) R 1 \quad R 1 R 2 /(R 1+R 2) R 1]+} \\
& {\left[\begin{array}{ll}
0 & -R 1 R 2 /(R 1+R 2) R 1
\end{array}\right]}
\end{aligned}
$$

These are the final A, B, C and D matrices according to the below structure. 
Structure: $\left\{\begin{array}{l}\dot{x}=A x+B u \\ y=C x+D u\end{array}\right.$

System Matrices:

$$
\begin{aligned}
& A=\left[\begin{array}{ccc}
-1 /\left(R_{1}+R_{2}\right) C_{1} & 1 /\left(R_{1}+R_{2}\right) C_{1} & 1 / C_{1}-R_{1} /\left(R_{1}+R_{2}\right) C_{1} \\
1 /\left(R_{1}+R_{2}\right) C_{2} & -1 /\left(R_{1}+R_{2}\right) C_{2} & R_{1} /\left(R_{1}+R_{2}\right) C_{2} \\
-1 / L+R_{1} /\left(R_{1}+R_{2}\right) L & -R_{1} /\left(R_{1}+R_{2}\right) L & -R_{L}-R_{1} R_{1} /\left(R_{1}+R_{2}\right) L
\end{array}\right] \\
& B=\left[\begin{array}{cc}
0 & -1 / C_{1}+R_{1} /\left(R_{1}+R_{2}\right) C_{1} \\
0 & -R_{1} /\left(R_{1}+R_{2}\right) C_{2} \\
1 / L & R_{1} R_{2} /\left(R_{1}+R_{2}\right) L
\end{array}\right] \\
& C=[1-R 1 /(R 1+R 2) R 1 \quad R 1 /(R 1+R 2) R 1 \quad R 1 R 2 /(R 1+R 2) R 1] \\
& D=[0 \quad-R 1 R 2 /(R 1+R 2) R 1]
\end{aligned}
$$

\subsection{Controllers used to control the Buck Converter:}

The control methods are applied to maintain the stable output voltage from the buck converter. The controlling of buck converter can be accomplished by using different controllers. In our thesis we use the following controller's Polynomial Controller, and Proportional Integral Derivative [PID] Controller.

These controller methods compare the actual output voltage with respect to the reference voltage. The difference between these voltages will lead the control element to attenuate the variations of the output voltage to the fixed reference voltage, this is known as voltage regulation.

\subsection{Get average system:}

The time averaged system can be derived as a weighting averaged of the two models. The averaged system ' $s$ ' average, system $s_{1}$ with voltage source and system $s_{2}$ without voltage 
source. Assuming the duty cycle with ' $d$ ', it can be seen that the output voltage of the converter varies linearly with the duty cycle for a given input voltage.

As the duty cycle $d$ is equal to the ratio between ton and the period $T$, it cannot be more than 1 . Therefore this is why this converter is referred to as step-down converter. and then it has

$$
s_{\text {average }}=d s_{1}+(1-d) s_{2}
$$

As it can be observed that $A B C D$ matrix of the two systems, that $A C D$ matrices are the same while the $B$ matrix are different in the ON and OFF states.

So only need to take the average value of $B$ matrix.

Where $\mathrm{B}_{1}=\left[\begin{array}{cc}0 & -1 / C_{1}+R_{1} /\left(R_{1}+R_{2}\right) C_{1} \\ 0 & -R_{1} /\left(R_{1}+R_{2}\right) C_{2} \\ 1 / L & R_{1} R_{2} /\left(R_{1}+R_{2}\right) L\end{array}\right] \quad$ and $\mathrm{B}_{2}=\left[\begin{array}{cc}0 & -1+R_{1} /\left(R_{1}+R_{2}\right) \\ 0 & -R_{1} /\left(R_{1}+R_{2}\right) \\ 0 & R_{1} R_{2} /\left(R_{1}+R_{2}\right)\end{array}\right]$

Average system equation, $\quad B=d\left(B_{11}+B_{12}\right)+(1-d)\left(B_{21}+B_{22}\right)$

Now substituting the below $B_{11}, B_{12}, B_{21}$ and $B_{22}$ matrices in above equation 19 , they are

$$
\mathrm{B}_{11}=\left[\begin{array}{cc}
0 & 0 \\
0 & 0 \\
1 / L & 0
\end{array}\right] \quad \mathrm{B}_{12}=\left[\begin{array}{cc}
0 & -1 / C_{1}+R_{1} /\left(R_{1}+R_{2}\right) C_{1} \\
0 & -R_{1} /\left(R_{1}+R_{2}\right) C_{2} \\
0 & R_{1} R_{2} /\left(R_{1}+R_{2}\right) L
\end{array}\right] \quad \mathrm{B}_{21}=\left[\begin{array}{ll}
0 & 0 \\
0 & 0 \\
0 & 0
\end{array}\right] \quad \mathrm{B}_{22}=\left[\begin{array}{cc}
0 & -1+R_{1} /\left(R_{1}+R_{2}\right) \\
0 & -R_{1} /\left(R_{1}+R_{2}\right) \\
0 & R_{1} R_{2} /\left(R_{1}+R_{2}\right)
\end{array}\right]
$$

Substituting the above $B_{11}, B_{12}, B_{21} a n d B_{22}$, Matrix's in the above equation 19, then

$$
\mathrm{B}=\mathrm{d}\left[\left[\begin{array}{cc}
0 & 0 \\
0 & 0 \\
1 / L & 0
\end{array}\right]+\left[\begin{array}{cc}
0 & -1 / C_{1}+R_{1} /\left(R_{1}+R_{2}\right) C_{1} \\
0 & -R_{1} /\left(R_{1}+R_{2}\right) C_{2} \\
0 & R_{1} R_{2} /\left(R_{1}+R_{2}\right) L
\end{array}\right]\right)\left(\begin{array}{c}
V_{S} \\
I_{0}
\end{array}\right)+
$$




$$
\begin{gathered}
\left.(1-\mathrm{d})\left[\begin{array}{ll}
0 & 0 \\
0 & 0 \\
0 & 0
\end{array}\right]+\left[\begin{array}{cc}
0 & -1+R_{1} /\left(R_{1}+R_{2}\right) \\
0 & -R_{1} /\left(R_{1}+R_{2}\right) \\
0 & R_{1} R_{2} /\left(R_{1}+R_{2}\right)
\end{array}\right]\right)\left(\begin{array}{c}
V_{S} \\
I_{0}
\end{array}\right] \\
\left.=\mathrm{d}\left[\begin{array}{cc}
0 & 0 \\
0 & 0 \\
V_{S} / L & 0
\end{array}\right]+\left[\begin{array}{cc}
0 & -I_{0} / C_{1}+R_{1} /\left(R_{1}+R_{2}\right) C_{1} \\
0 & -R_{1} I_{0} /\left(R_{1}+R_{2}\right) C_{2} \\
0 & R_{1} R_{2} I_{0} /\left(R_{1}+R_{2}\right) L
\end{array}\right]\right)+(1-\mathrm{d})\left[\left[\begin{array}{ll}
0 & 0 \\
0 & 0 \\
0 & 0
\end{array}\right]+\left[\begin{array}{cc}
0 & -I_{0} / C_{1}+R_{1} /\left(R_{1}+R_{2}\right) C_{1} \\
0 & -R_{1} I_{0} /\left(R_{1}+R_{2}\right) C_{2} \\
0 & R_{1} R_{2} I_{0} /\left(R_{1}+R_{2}\right) L
\end{array}\right]\right)
\end{gathered}
$$

After multiplying the above matrix with ' $\mathrm{d}$ ' and [1-d], then simplifying the above equation, by taking 'd' adjacent matrix as common,

$$
\begin{aligned}
& =\left[\begin{array}{cc}
0 & 0 \\
0 & 0 \\
V_{S} / L & 0
\end{array}\right] \mathrm{d}+\left[\begin{array}{cc}
0 & -I_{0} / C_{1}+R_{1} /\left(R_{1}+R_{2}\right) C_{1} \\
0 & -R_{1} I_{0} /\left(R_{1}+R_{2}\right) C_{2} \\
0 & R_{1} R_{2} I_{0} /\left(R_{1}+R_{2}\right) L
\end{array}\right] \\
& =\left[\begin{array}{cc}
0 & -I_{0} / C_{1}+R_{1} /\left(R_{1}+R_{2}\right) C_{1} \\
0 & -R_{1} I_{0} /\left(R_{1}+R_{2}\right) C_{2} \\
V_{S} / L & R_{1}+R_{2} I_{0} /\left(R_{1}+R_{2}\right) L
\end{array}\right]\left(\begin{array}{l}
d \\
I_{0}
\end{array}\right)
\end{aligned}
$$

The averaged system is: $\quad\left\{\begin{array}{l}\dot{x}=A x+B u \\ y=C x+D u\end{array}\right.$

And the continuous-time system is,

$$
\left[\begin{array}{l}
\frac{d}{d t} u_{C 1} \\
\frac{d}{d t} u_{C 2} \\
\frac{d}{d t} i_{L}
\end{array}\right]=\left[\begin{array}{cc}
0 & -1 / C_{1}+R_{1} /\left(R_{1}+R_{2}\right) C_{1} \\
0 & -R_{1} /\left(R_{1}+R_{2}\right) C_{2} \\
-1 / L & R_{1}+R_{2} /\left(R_{1}+R_{2}\right) L
\end{array}\right]\left[\begin{array}{c}
u_{C 1} \\
u_{C 2} \\
i_{L}
\end{array}\right]+\left[\begin{array}{cc}
0 & -I_{0} / C_{1}+R_{1} /\left(R_{1}+R_{2}\right) C_{1} \\
0 & -R_{1} I_{0} /\left(R_{1}+R_{2}\right) C_{2} \\
V_{S} / L & \left(R_{1}+R_{2}\right) I_{0} /\left(R_{1}+R_{2}\right) L
\end{array}\right]\left[\begin{array}{l}
d \\
I_{0}
\end{array}\right]
$$

Since $B$ is the average result of the switched system were the input voltage, capacitor, resistor and inductor values are fixed. Here the $B$ value depends on the duty cycle $d$ and $i_{0}$. So the system we get is linear with the duty cycle [control signal] $d$ and $i_{0}$. 


\section{Chapter 4. Polynomial Pole Placement Controller:}

This section explains designing of a Polynomial Controller with Pole Placement for the third order buck plant. We used discrete time case because, the implementation should be done in a computer.

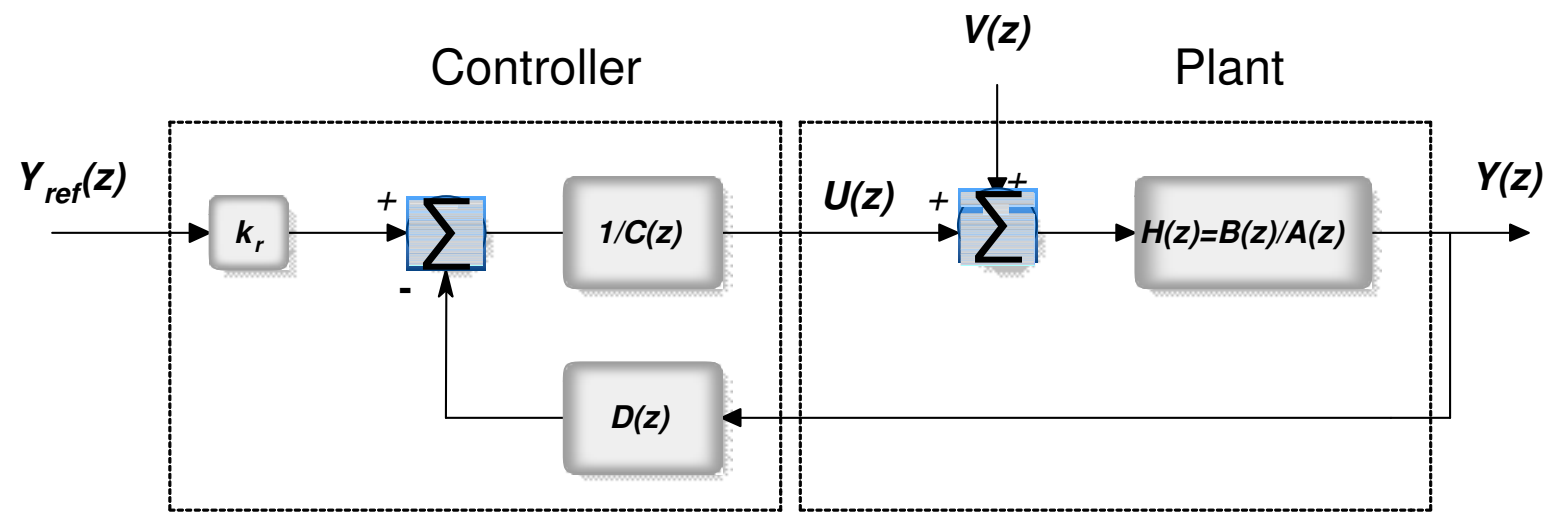

Figure 9: Buck Converter System H (Z) with Polynomial Controller [7]

To develop controller structure form the pole placement controller, which gives the closed system of exact poles defined by own dynamics characteristics [7].

This does not include any direct comparison between the set-point and actual value according to rules of error. But it exposes the set-point value and actual value for the more individual treatment doing so, is $C(z)$ and $D(z)$ polynomials with the delay operator $z^{-1}$ [7]. As our buck converter plant is of third order we have consider five parameters, from the design and output of the second order polynomial pole placement controller [5] i.e., $c_{1}, c_{2}, d_{0}, d_{1}$ and $d_{2}$ to achieve necessary design freedom.

$$
\begin{aligned}
& C(z)=1+c_{1} z^{-1}+c_{2} z^{-2} \\
& D(z)=d_{0}+d_{1} z^{-1}+d_{2} z^{-2}
\end{aligned}
$$

The determined equation depicting from the above figure 9 is

$$
C(z) \cdot U(z)=K_{r} \cdot Y_{r e f}(z)-D(z) \cdot Y(z)
$$

Which by above equations 22 and $\mathbf{2 3}$, the interpretation of $z^{-1}$ the delay operator gives the differential equation 
$u(k)=K_{r} \cdot Y_{r e f}(k)-c_{1} y(k-1)-c_{2} y(k-2)-d_{0} y(k)-d_{1} y(k-1)-d_{2} y(k-2)$

The controller relationship in the equation $\mathbf{2 4}$, defines the control signal at the $k^{\text {th }}$ sampling time as a function of the set-point and output measurement.

The closed circuit transfer function in figure 9, is given by,

$$
\frac{Y(z)}{Y_{r e f}(z)}=K_{r} \cdot \frac{H(z)}{C(z)+H(z) \cdot D(z)}
$$

We know that $H(z)=\frac{B(z)}{A(z)}$

The $H(Z)$ is a discredited time continuous transfer function, and the discretion is performed with zero order hold circuit with the help of Matlab c2d function.

Substituting the $H(z)$ in equation $\mathbf{2 5}$, we get as shown below,

$$
\frac{Y(z)}{Y_{r e f}(z)}=K_{r} \cdot \frac{B(z)}{A(z) C(z)+B(z) \cdot D(z)}
$$

The design comprises of the $C$ and $D$ polynomials.

The characteristic polynomial is named as $P(z)$.

Now $P(z)=A(z) C(z)+B(z) \cdot D(z)$

Re-writing the equation $\mathbf{2 6}$, as shown below

$$
\frac{Y(z)}{Y_{r e f}(z)}=K_{r} \cdot \frac{B(z)}{P(z)}
$$


The complete analytical designing procedure is as follows.

Choose the plant of order. Here we have $3^{\text {rd }}$ order Buck converter plant.

Plant $H(z)=\frac{B(z)}{A(z)}=\frac{b 2 z^{2}+b 2 z+b 3}{z^{3}+a 1 z^{2}+a 2 z+a 3}=\frac{b 1 z^{-1}+b 2 z^{-2}+z^{-3}}{1+a 1 z^{-1}+a 2 z^{-2}+a 3 z^{-3}}$

The characteristic polynomial equation is $P(z)=A(z) C(z)+B(z) \cdot D(z)$

Substitute $C(z)$ and $D(z)$ values in above equation $\mathbf{2 8}$, then

$C\left(z^{-1}\right)=\left(1+c_{1} z^{-1}+c_{2} z^{-2}\right)$

$D\left(z^{-1}\right)=\left(d_{0}+d_{1} z^{-1}+d_{2} z^{-2}\right)$

$P(z)=$

$\left(1+a 1 z^{-1}+a 2 z^{-2}+a 3 z^{-3}\right)\left(1+c_{1} z^{-1}+c_{2} z^{-2}\right)+\left(b 1 z^{-1}+b 2 z^{-2}+z^{-3}\right)\left(d_{0}+d_{1} z^{-1}+d_{2} z^{-2}\right)$

(29)

Multiplying the above equation on RHS [Right Hand Side] gives

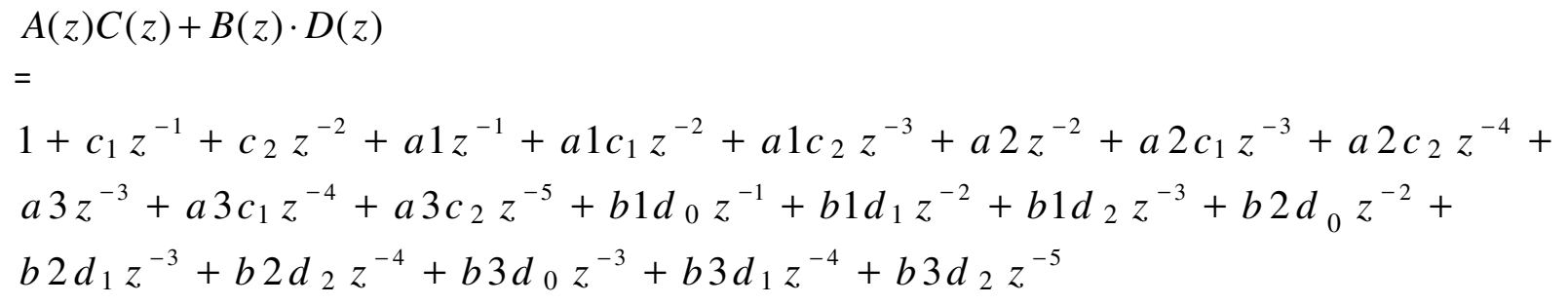

Take $z^{-1}, z^{-2}, z^{-3}, z^{-4}, z^{-5}$ as common in above equation 29 . The resultant equation is

$$
\begin{aligned}
& 1+\left(c_{1}+a 1+b 1 d_{0}\right) z^{-1}+\left(c_{2}+a 1 c_{1}+a 2+b 1 d_{1}+b 2 d_{0}\right) z^{-2}+\left(a 1 c_{2}+a 2 c_{1}+a 3+\right. \\
& \left.b 1 d_{2}+b 2 d_{1}+b 3 d_{0}\right) z^{-3}+\left(a 2 c_{2}+a 3 c_{1}+b 2 d_{2}+b 3 d_{1}\right) z^{-4}+\left(a 3 c_{2}+b 3 d_{2}\right) z^{-5}
\end{aligned}
$$


By choosing the controller parameters, we can freely design the closed loop system pole placement.

Finding poles $\mathrm{P} 1, \mathrm{P} 2, \mathrm{P} 3, \mathrm{P} 4$ and $\mathrm{P} 5$ in terms of $q 1, q 2, q 3, q 4$ and $q 5$

$$
\begin{aligned}
& P(z)=\left(1-q 1 z^{-1}\right)\left(1-q 2 z^{-1}\right)\left(1-q 3 z^{-1}\right)\left(1-q 4 z^{-1}\right)\left(1-q 5 z^{-1}\right)= \\
& 1+p 1 z^{-1}+p 2 z^{-2}+p 3 z^{-3}+p 4 z^{-4}+p 5 z^{-5}
\end{aligned}
$$

Finding the values $\mathrm{P} 1, \mathrm{P} 2, \mathrm{P} 3, \mathrm{P} 4$, and $\mathrm{P} 5$.

$$
\begin{aligned}
& P 1=-q 5-q 4-q 3-q 2-q 1 \\
& P 2=q 4 q 5+q 3 q 5+q 3 q 4+q 2 q 5+q 2 q 4+q 2 q 3+q 1 q 5+q 1 q 4+q 1 q 3+q 1 q 2 \\
& P 3=-q 3 q 4 q 5-q 2 q 4 q 5-q 2 q 3 q 5-q 2 q 3 q 4-q 1 q 4 q 5- \\
& q 1 q 3 q 5-q 1 q 3 q 4-q 1 q 2 q 5-q 1 q 2 q 5-q 1 q 2 q 4-q 1 q 2 q 3 \\
& P 4=q 2 q 3 q 4 q 5+q 1 q 3 q 4 q 5+q 1 q 2 q 4 q 5+q 1 q 2 q 3 q 5+q 1 q 2 q 3 q 4 \\
& P 5=-q 1 q 2 q 3 q 4 q 5
\end{aligned}
$$

For the remaining derivation please go to the Appendix 2.

Finally we get the $\mathrm{F}, \mathrm{M}$ and $\mathrm{N}$ matrices.

Form the simplified equation in Appendix-2, let the matrices be,

$$
M=\left(\begin{array}{ccccc}
1 & 0 & b 1 & 0 & 0 \\
a 1 & 1 & b 2 & b 1 & 0 \\
a 2 & a 1 & b 3 & b 2 & b 1 \\
a 3 & a 2 & 0 & b 3 & b 2 \\
0 & a 3 & 0 & 0 & b 3
\end{array}\right), F=\left(\begin{array}{c}
c 1 \\
c 2 \\
d 0 \\
d 1 \\
d 2
\end{array}\right) \text { and }
$$




$$
N=\left(\begin{array}{c}
-a 1+(-q 5-q 4-q 3-q 2-q 1) \\
-a 2+q 4 q 5+q 3 q 5+q 3 q 4+q 2 q 5+q 2 q 4+q 2 q 3+q 1 q 5+q 1 q 4+q 1 q 3+q 1 q 2 \\
-a 3+(-q 3 q 4 q 5-q 2 q 4 q 5-q 2 q 3 q 5-q 2 q 3 q 4-q 1 q 4 q 5- \\
q 1 q 3 q 5-q 1 q 3 q 4-q 1 q 2 q 5-q 1 q 2 q 5-q 1 q 2 q 4-q 1 q 2 q 3) \\
q 2 q 3 q 4 q 5+q 1 q 3 q 4 q 5+q 1 q 2 q 4 q 5+q 1 q 2 q 3 q 5+q 1 q 2 q 3 q 4 \\
-q 1 q 2 q 3 q 4 q 5
\end{array}\right)
$$

The polynomials $c_{1}, c_{2}, d_{0}, d_{1}$ and $d_{2}$ are obtained by $F=M^{-1} \times N$

The analytical calculation of $5 \times 5$ matrix is pretty tough, so we used Matlab for a numerical solution. The values for poles q1, q2, q3, q4 and q5 are considered to be $0.5,0.5,0.5,0.5$ and 0.5 respectively.

Here we had taken 0.5 value for poles because the output is not stable for the remaining combinations mentioned below for both the PID, PD and Polynomial controllers. And the resulted graphs for Polynomial Pole Placement are provided in the Appendix 3.

At $2 \times 3 e^{-6}$, the following pole combination are consider

$q 1=0.1, q 2=0.2, q 3=0.3, q 4=0.4, q 5=0.5$.

$q 1=0.3, q 2=0.4, q 3=0.5, q 4=0.6, q 5=0.7$ and

$q 1=0.5, q 2=0.5, q 3=0.5, q 4=0.5, q 5=0.5$

At $2 \times 3 \mathrm{e}^{-6}$, and poles $\mathrm{q} 1=0.5, \mathrm{q} 2=0.5, \mathrm{q} 3=0.5, \mathrm{q} 4=0.5, \mathrm{q} 5=0.5$, the result is better than the remaining combinations, so we consider these pole values for the PID, PD and Polynomial controllers.

And the below specified formula [8], for finding multiple poles resulted approximately same as above mentioned iterations. Here we have to find the sampling interval ' $h$ ' from the below formula, by substituting the bandwidth ' $\omega_{b}$ ' and number of samples ' $N$ ', the $N$ value varies from $10-20$ here we had considered $N=20$ and bandwidth $\omega_{b}=9 \mathrm{k}$, because to get the output value less than $5 \mu \mathrm{s}$. we can check the scanner- pole placement for a multiple poles table 16.1 from [8], shown below 


\begin{tabular}{|c|c|}
\hline$m$ & $Z=p$ \\
\hline 1 & 0.73 \\
\hline 2 & 0.61 \\
\hline 3 & 0.54 \\
\hline 4 & 0.49 \\
\hline 5 & 0.44 \\
\hline
\end{tabular}

Table 1: Pole Placement for Multiple poles.

$$
h=\left(\frac{2 \pi}{N \omega_{b}}\right)
$$

Were $\mathrm{h}=$ sampling interval,

$$
\begin{aligned}
& \mathrm{N}=20 \text {, and } \\
& \omega_{b}=\text { Bandwidth is } 9 \mathrm{k} .
\end{aligned}
$$

And the sampling interval $\mathrm{h}$ will be,

$$
\begin{gathered}
h=\left(\frac{2 \prod}{20 * 2 \prod * 9 k}\right) \\
h=4 e^{-6}
\end{gathered}
$$

So from the above formula we get the sampling interval as $4 e^{-6}$, which is used in the Polynomial pole placement Matlab code [Appendix 8], as sampling interval for the controller to obtain the reference voltage $3.3 \mathrm{v}$.

\subsection{Polynomial Controller Simulation:}

After obtaining the mathematical equations for Polynomial Controller, the next task is to build the system in Simulink and program the procedure in Matlab. The controller and plant is to be built same as the structure shown in the Figure 9. The polynomial control structure, as given in Figure 9, can be implemented in Simulink as presented in Figure 10. 


\subsection{Building the Controller block in Simulink:}

Here considering the output voltage as the input of controller and comparing it by the reference voltage as show in the below Figure 10.

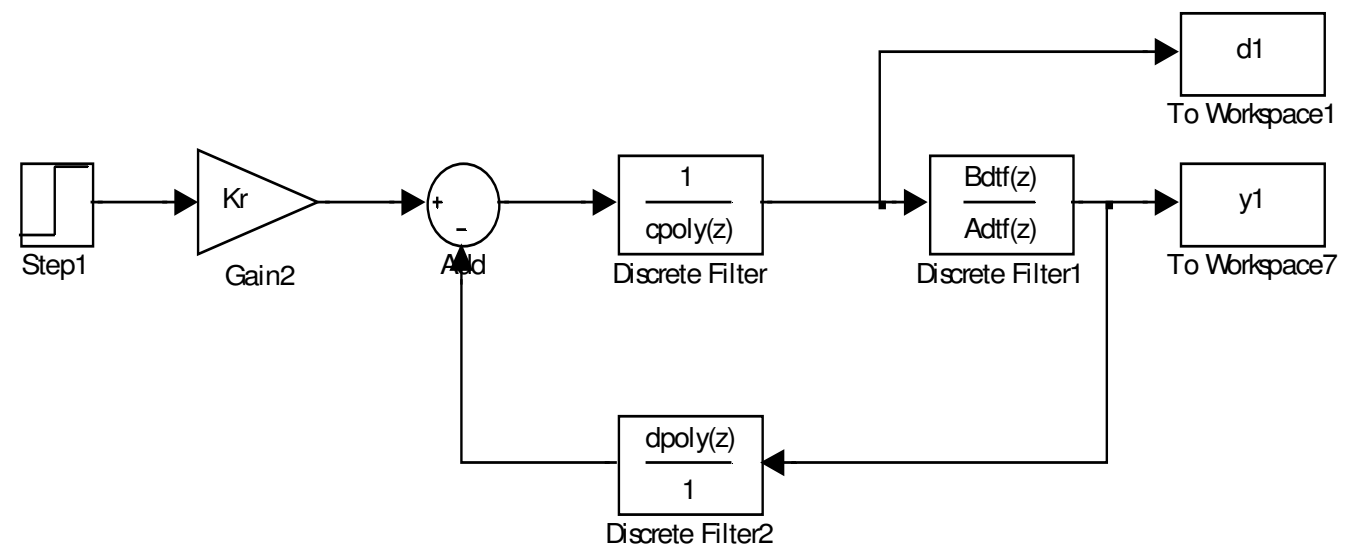

Figure 10: Polynomial Controller block

The block diagram Figure 10, depicts the discrete polynomial design which consists of 3 discrete filters such as cpoly, dpoly and the plant $\operatorname{Bdtf}(z) / \operatorname{Adtf}(z)$ were the $\operatorname{cpoly}(z)$ and $\operatorname{dpoly}(z)$ polynomial equations are $C(z)=1+c_{1} z^{-1}+c_{2} z^{-2}, D(z)=d_{0}+d_{1} z^{-1}+d_{2} z^{-2}$.

\subsection{Results of Polynomial Controller:}

These blocks diagrams are with the help of appropriate Matlab files to obtain the below resulted graphs. 


\subsubsection{Simulation results of the Continuous and Discrete Model:}

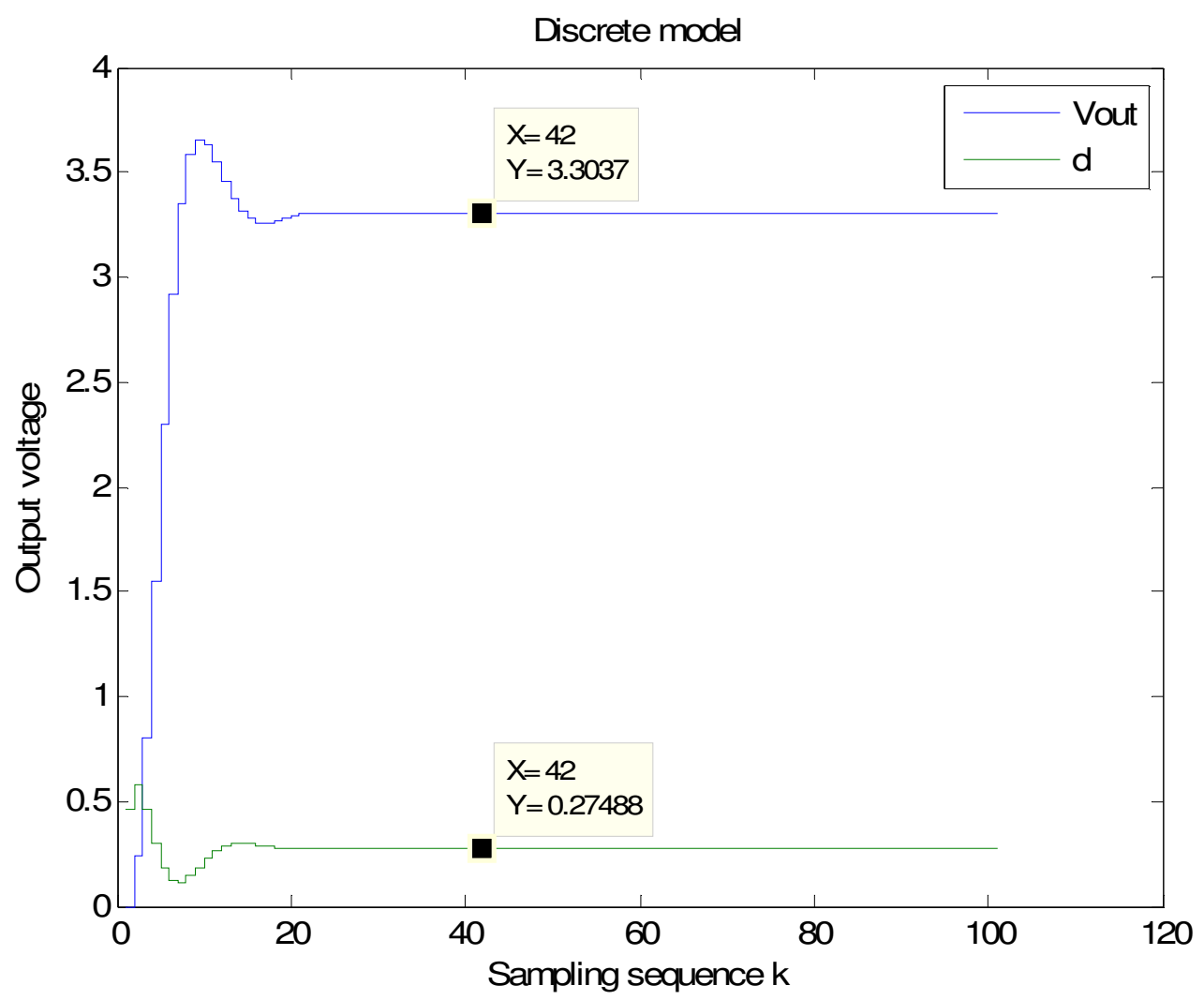

Figure 11: Discrete model system and controller output.

The simulation is an numerical illustration of the theory. And while designing the controller, the saturation limits should not be reached too much for a reasonable disturbance, and the simulation result satisfies it.

From the Figure 11, we can observe that, the output voltage of discrete system satisfies the required voltage and the output of the controller gets stable quickly [in 18 sample intervals] and remains stable. The desired output voltage [reference voltage 3.3V] by the simulations derived controller with a time continuous model.

The controller is also tested controlling the time continuous system, the average system model consists of the polynomial controller and a continuous system. For Simulation result, see Figure 11. 


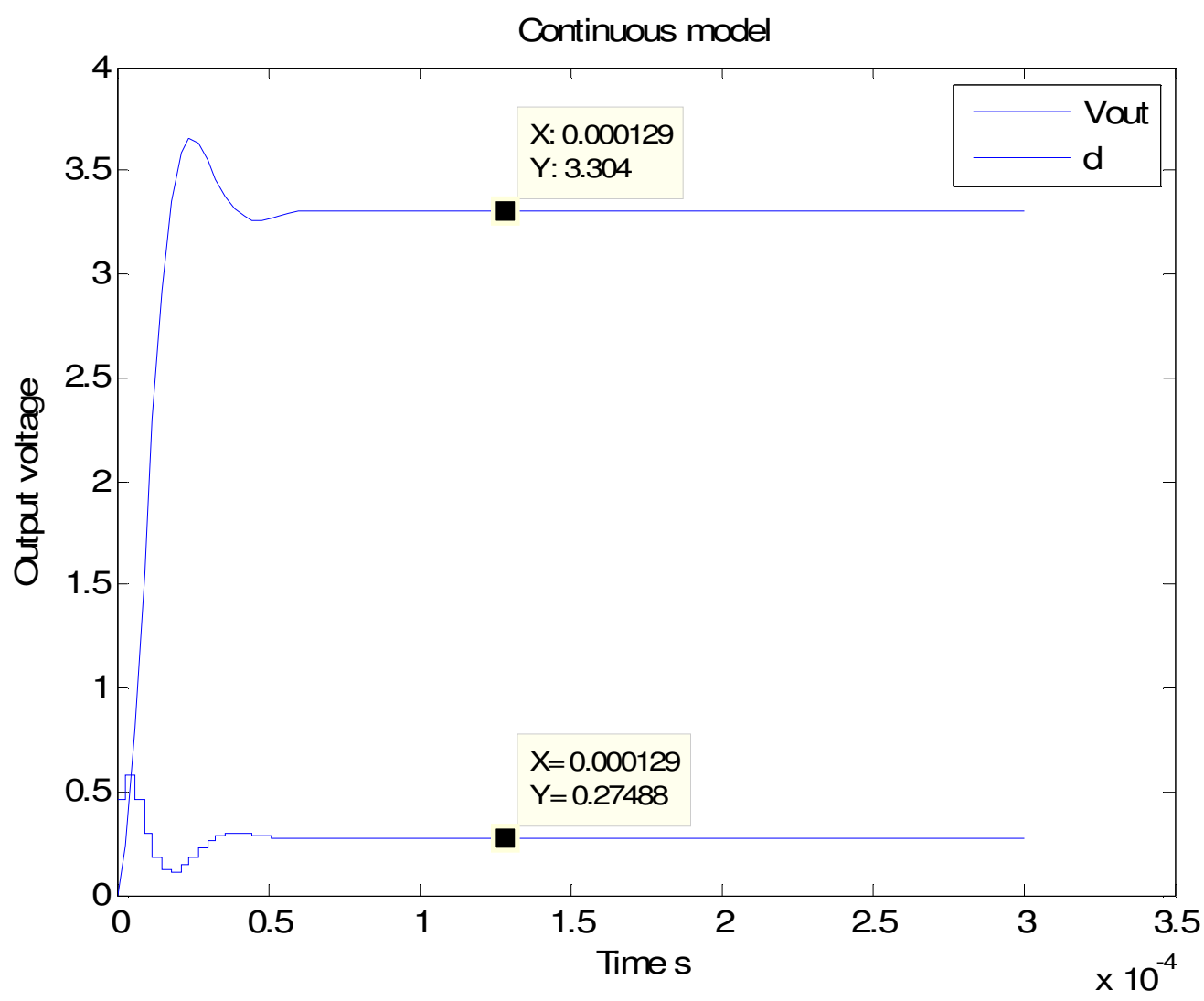

Figure 12: Continuous model system and controller output

Since the reference voltage is $3.3 \mathrm{~V}$, the output voltage can reach it very well. We can observe that the output voltage remains stable at $3.3 \mathrm{~V}$. The result shows that the continuous model of Polynomial Controller works perfectly.

The controller attains the output voltage to be stable quickly [in $50 \mu \mathrm{s}$ ] and remain the desired output voltage [reference voltage $3.3 \mathrm{~V}$ ]. It is very nearer to the polynomial controller theory. Figure 12.

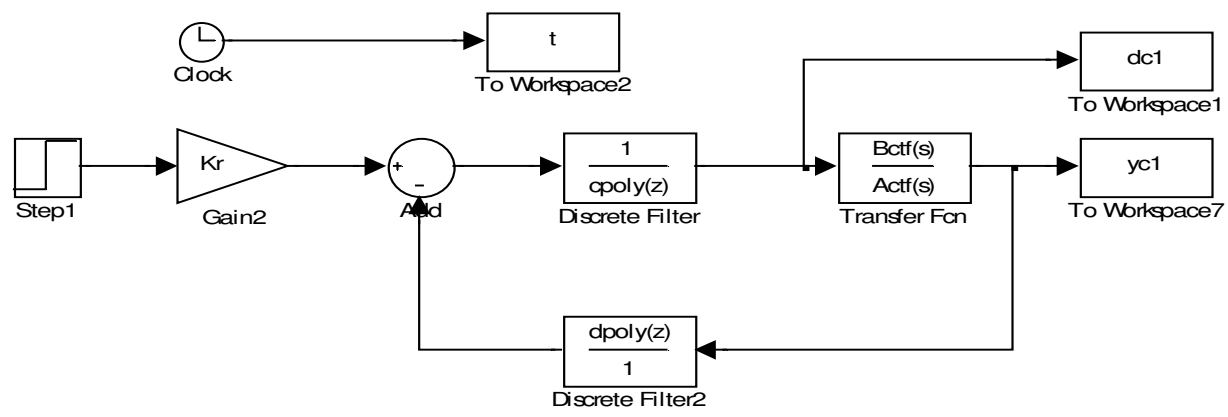

Figure 13: Continuous Time Model 


\subsubsection{Simulation results of Switched system without using Disturbance and Noise:}

The model is a switched time continuous model, which is an interesting part of our work. It investigates how good the controller works for this system with disturbance.

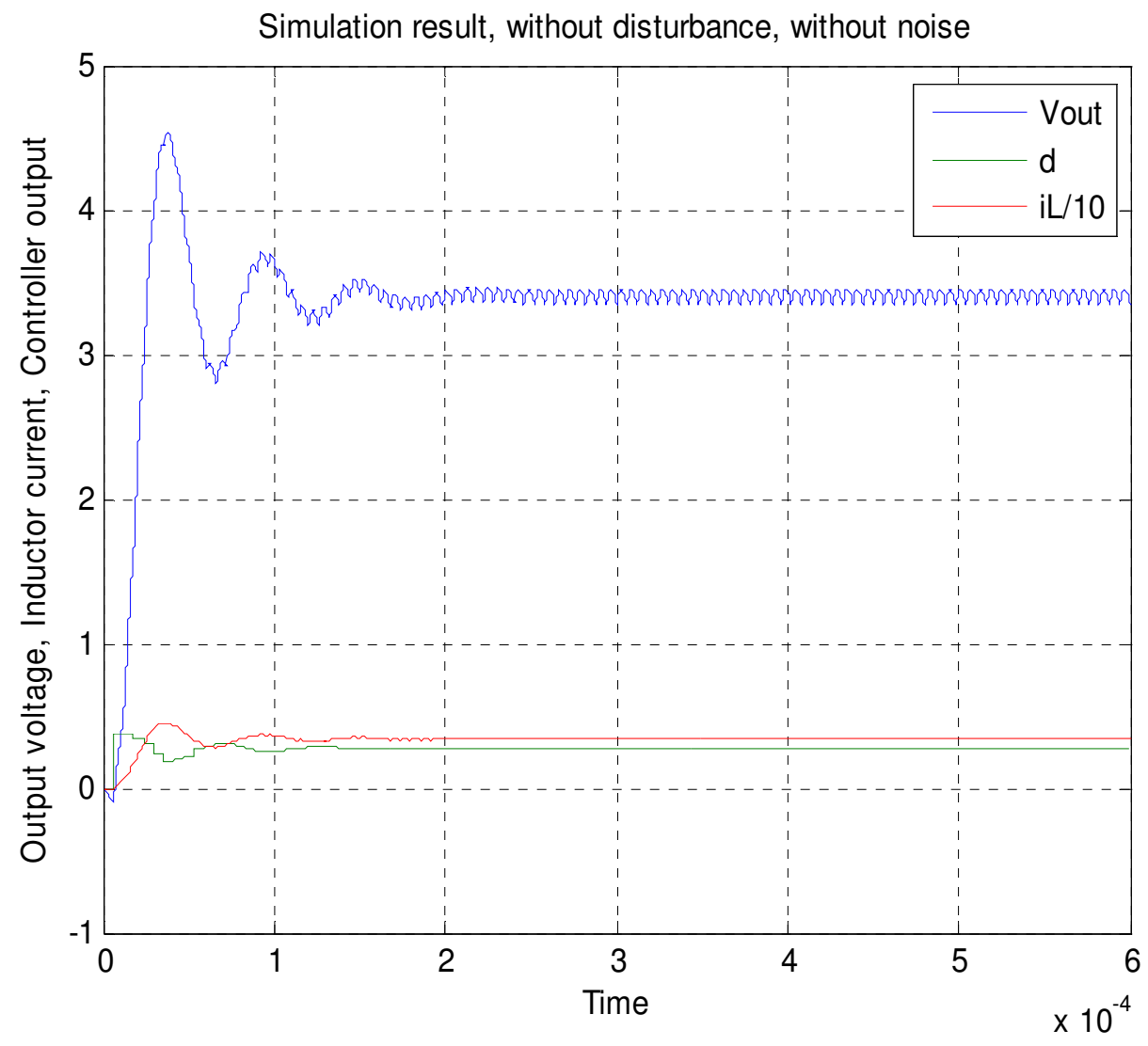

Figure 14: Simulation result, without disturbance, and noise.

It can be observe from Figure 14 that the output voltage is quite stable near $3.38 \mathrm{~V}$ which is very nearer to the given reference voltage i.e., $3.38 \mathrm{v}$.

As it can be observed from figure 14, that the output voltage of discrete system can satisfy the required voltage. And the controller output is also very stable near $0.2816=3.38 / 12$ [reference voltage divide input voltage], and remain the wanted output voltage [reference voltage 3.3V]. The simulation is according to the polynomial controller theory, so it is an illustration of the theory by numerical calculations.

When designing the controller, it should not hit the saturation limits too much for a reasonable disturbance. And the simulation result satisfies it. 
Here Figure 14 is the resulted graph of the block diagram shown below Figure 15.

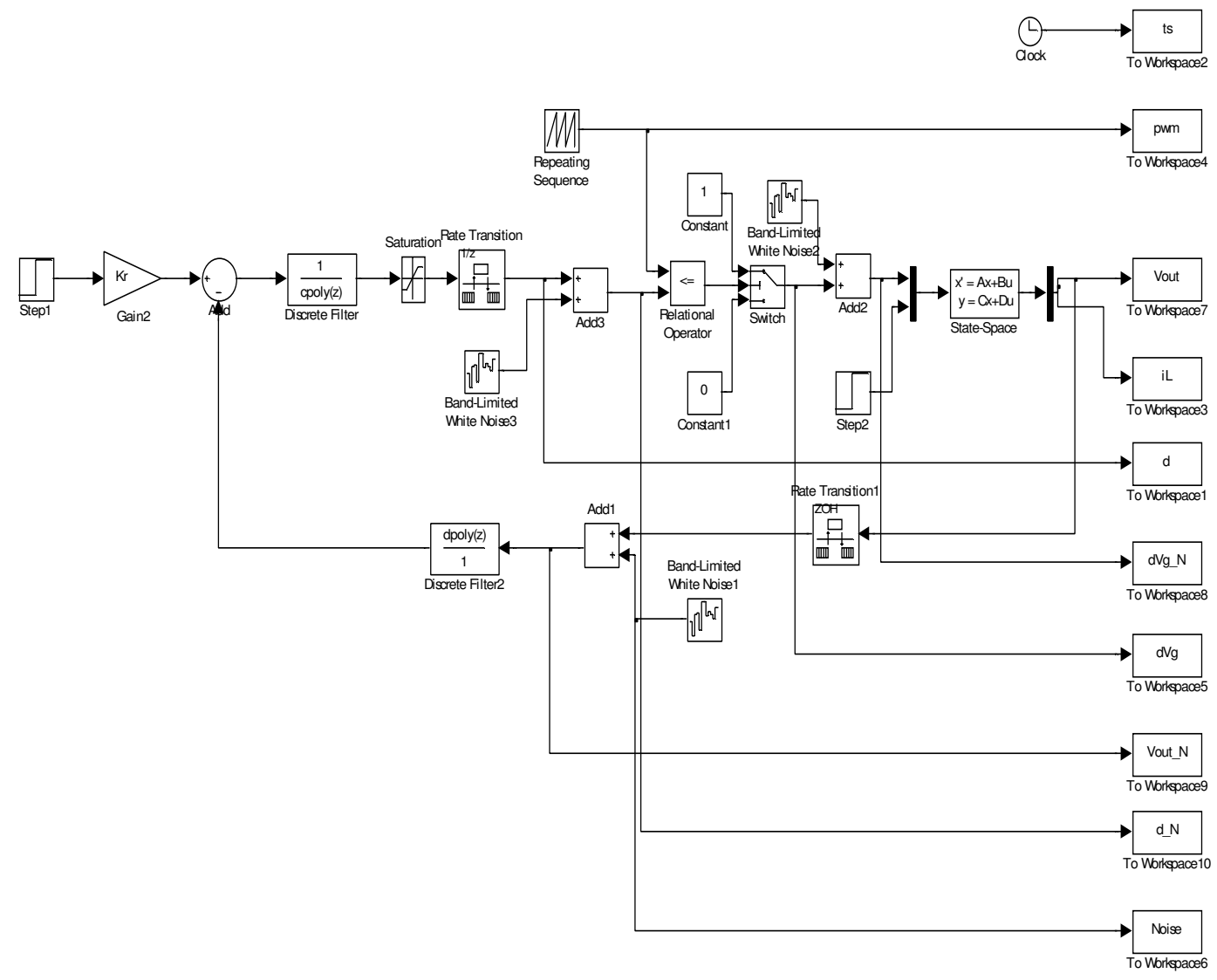

Figure 15: PWM Model with Controller

\subsubsection{Simulation result without Disturbance and with Noise:}

In the realistic case, the circuit has noise, at the switch with respect to high frequency switching measurement noise and so on.

Here, adding some band-limited white noise to simulate it, and the noise magnitude is between -0.2 and 0.2 , and the noise frequency is $33 \mathrm{MHz}$.

The frequency is a very rough estimation based on a measurement, see Fig. 16. And the measurements are introduced by the switching, but use it the whole time 


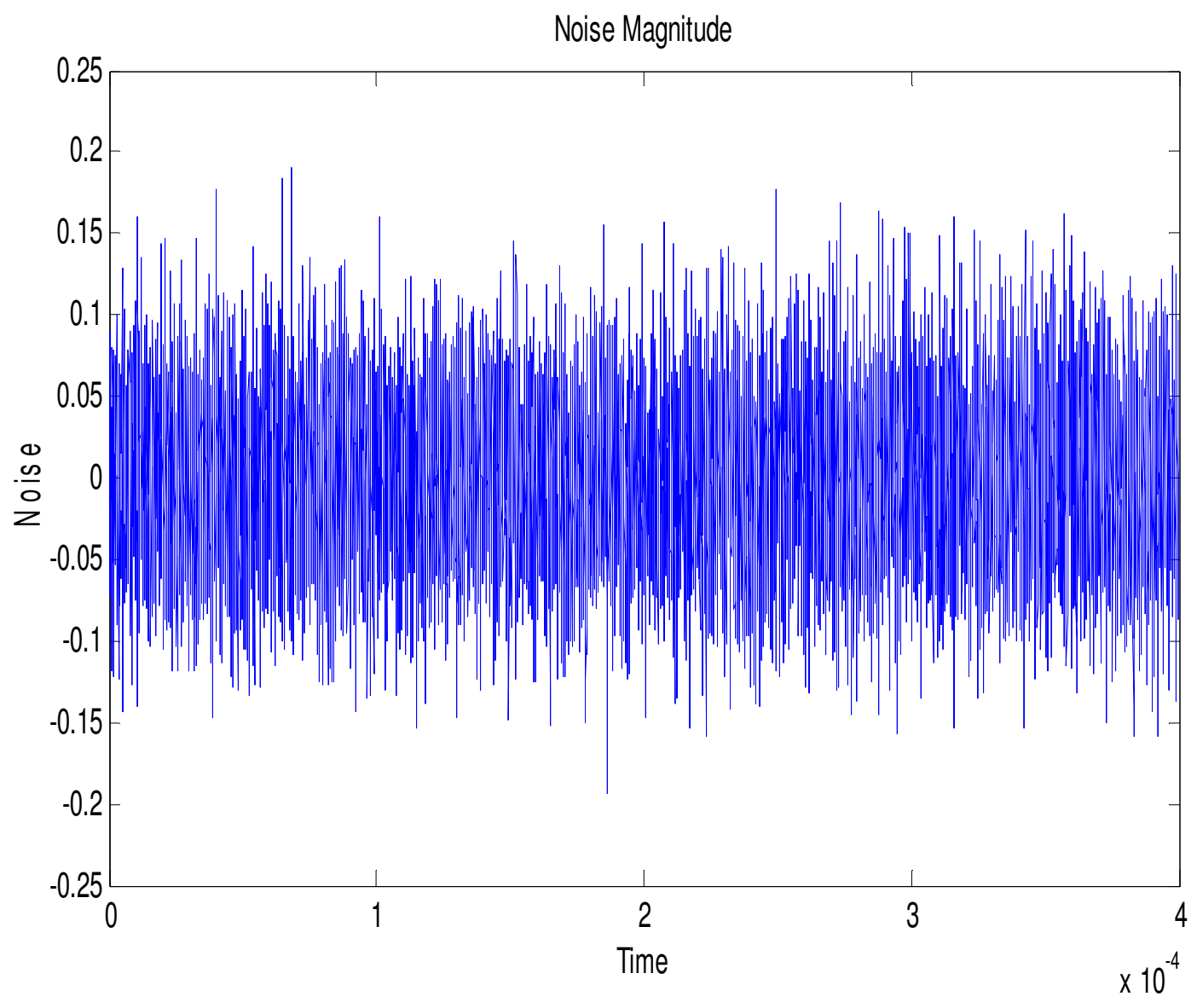

Figure 16: Noise Magnitude

The noise applied in this simulation is satisfactory, Thus the same amount of noise is considered in the simulation. This noise is sum up with the output voltage as the measurement noise. And the output voltage with noise is shown in Figure 17. 


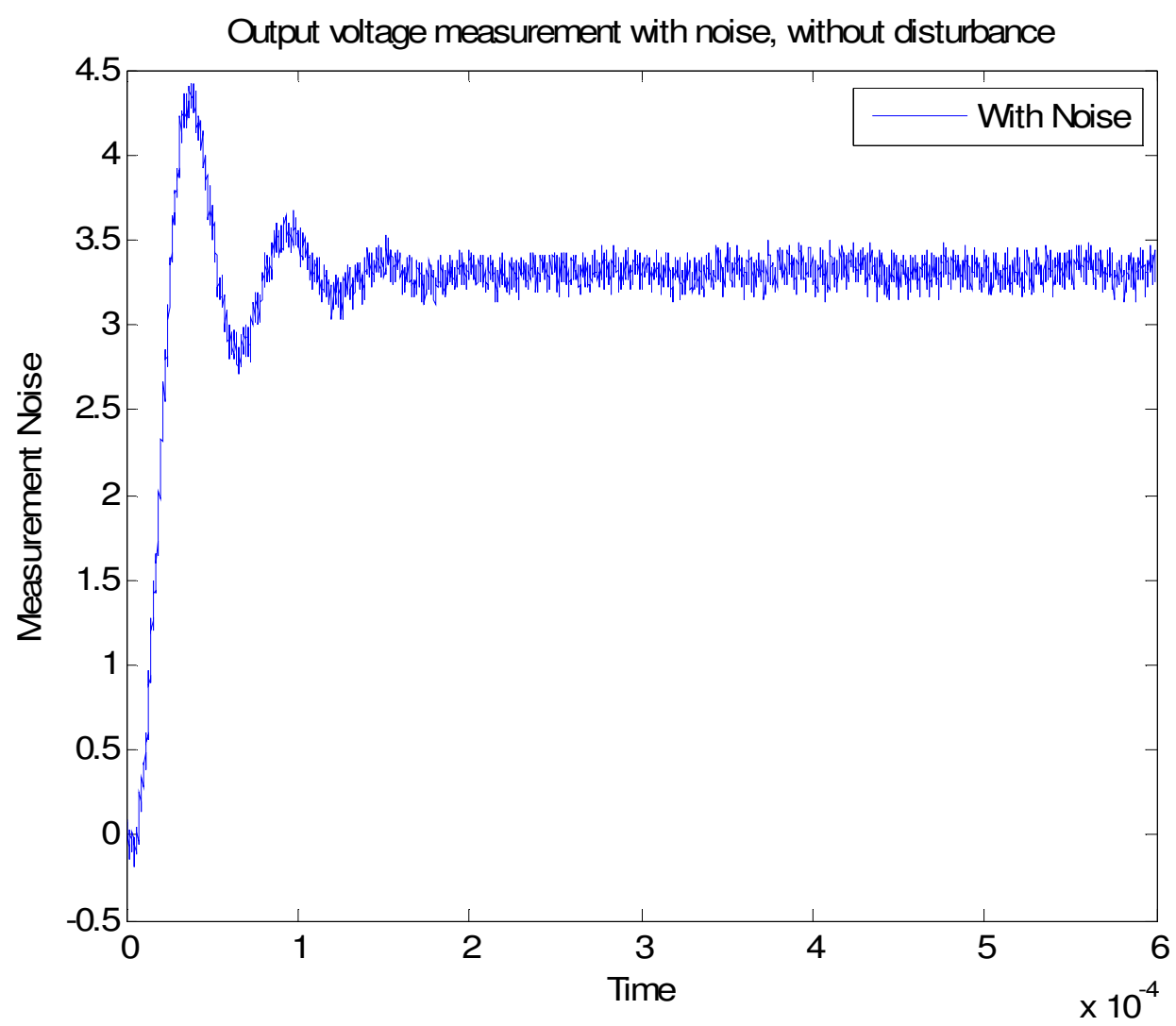

Figure 17: Simulation result, with noise and without disturbance.

It can be observable that after adding some amount of noise, the output is still stable. But the noise is not huge, it has some effect on the stability of controller. In this range 3.0V-3.5V the output does not get stable. The result shows that the controller with noise is less stable when compared to the controller without noise.

For the noise position block diagram, see figure 15.

\subsubsection{Simulation result with Disturbance and without Noise:}

In this model, the current source is applied as the load, when the output current abruptly changes, but there is some sort of disturbance in the output voltage.

To notice, the disturbance and check whether the Polynomial Controller has ability to turn back the output voltage value equal to reference voltage value. 


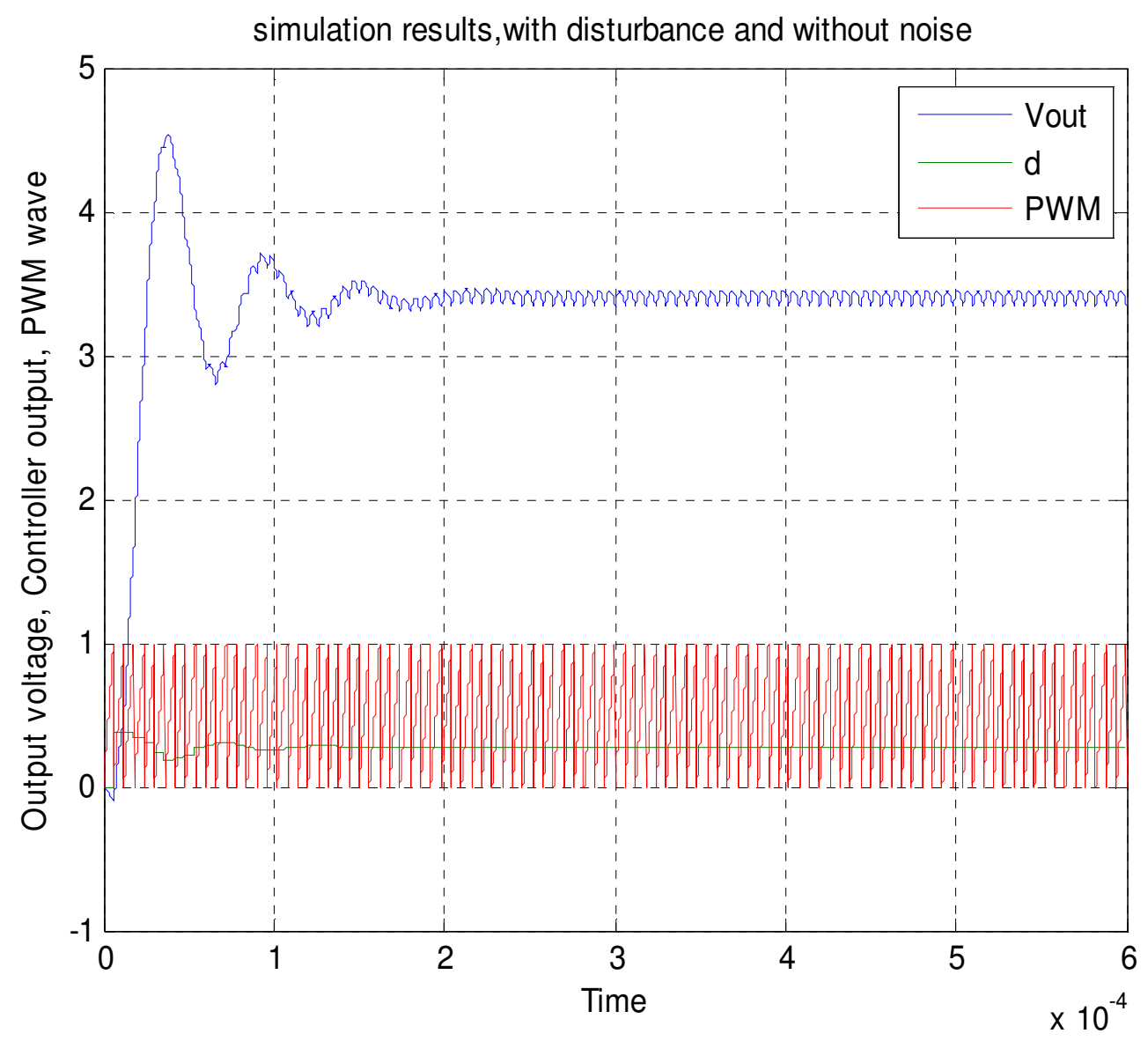

Figure 18: Simulation result, with disturbance, without noise

The output voltage under control is about $3.309 \mathrm{~V}$ it is quite close to the reference voltage 3.3V; it means that the controller is in good state.

At the 60th sampling interval [180 $\mu \mathrm{s}$ ] changing the current at the load from $5 \mathrm{~A}$ to $15 \mathrm{~A}$, the output voltage falls from $3.309 \mathrm{~V}$ to $2.883 \mathrm{~V}$ but it recover to the stable voltage $3.355 \mathrm{~V}$ shortly [in 10 sample intervals].

The drop percentage is (3.309-2.883)/3.309 $=12 \%$, which is adequate for the good performance. When the load current changes abruptly, the controller controls the output voltage and recovers to the stable voltage soon. 


\subsubsection{Simulation result with disturbance and with noise:}

Adding the same noise like what it is done in above section 4.3.4 for simulation result, see Figure 19.

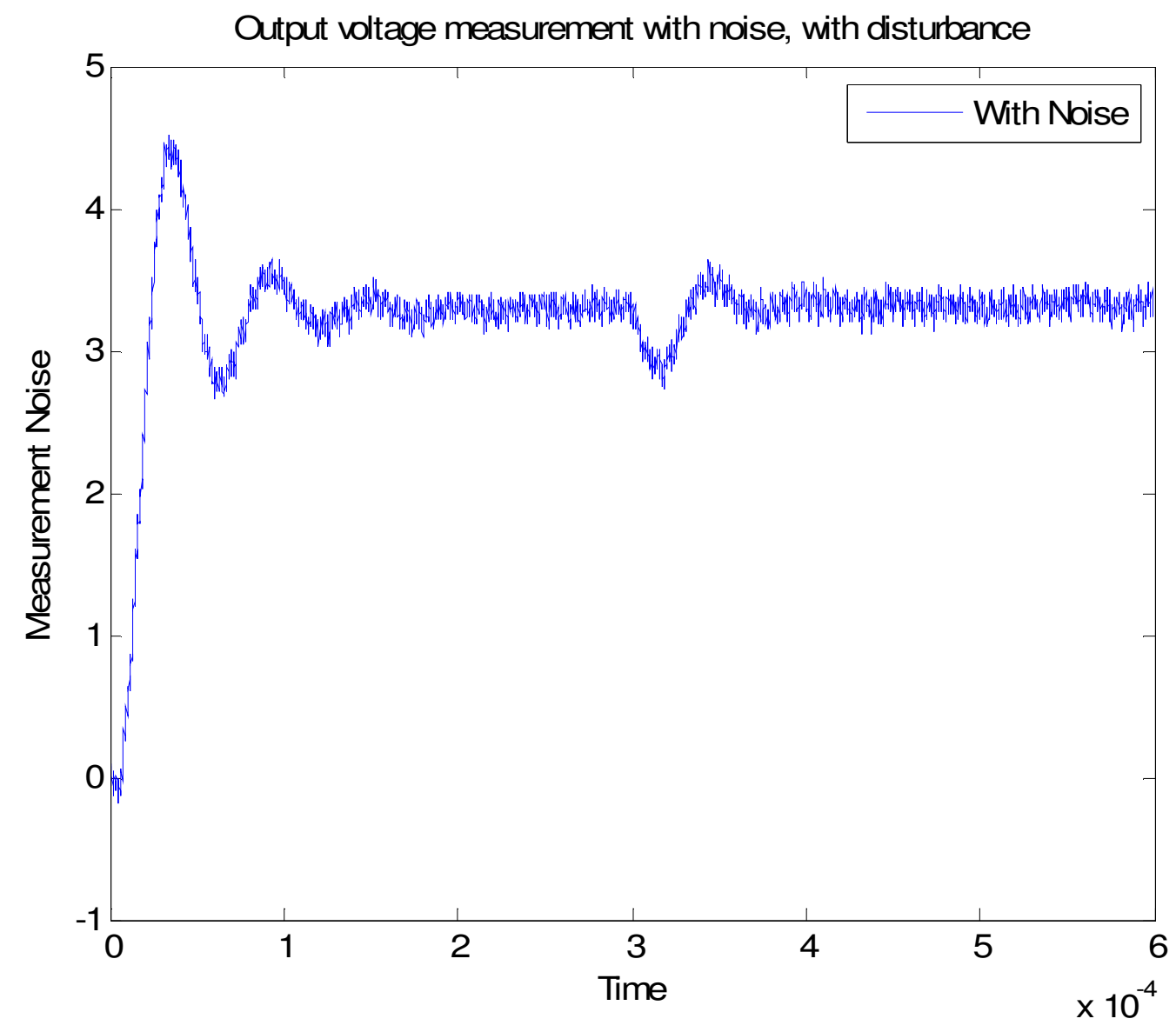

Figure 19: Simulation result, with Disturbance and Noise

As it can be observable, after summing the noise, the output voltage is stable. And the output voltage is closer to the reference voltage were the controller output is close to 0.280 .

\subsubsection{Simulation results with high value resistors:}

If we consider all the three resistors $R_{L}, R_{1}$ and $R_{2}$ with large values [100 $\mathrm{e}^{-3}$, with the Polynomial Controller, after running the simulation we can observe the controller quality from the below graph in Figure 20. We have also simulated with slight change in capacitors and inductors. We have observed that there is no noticeable change in results. 


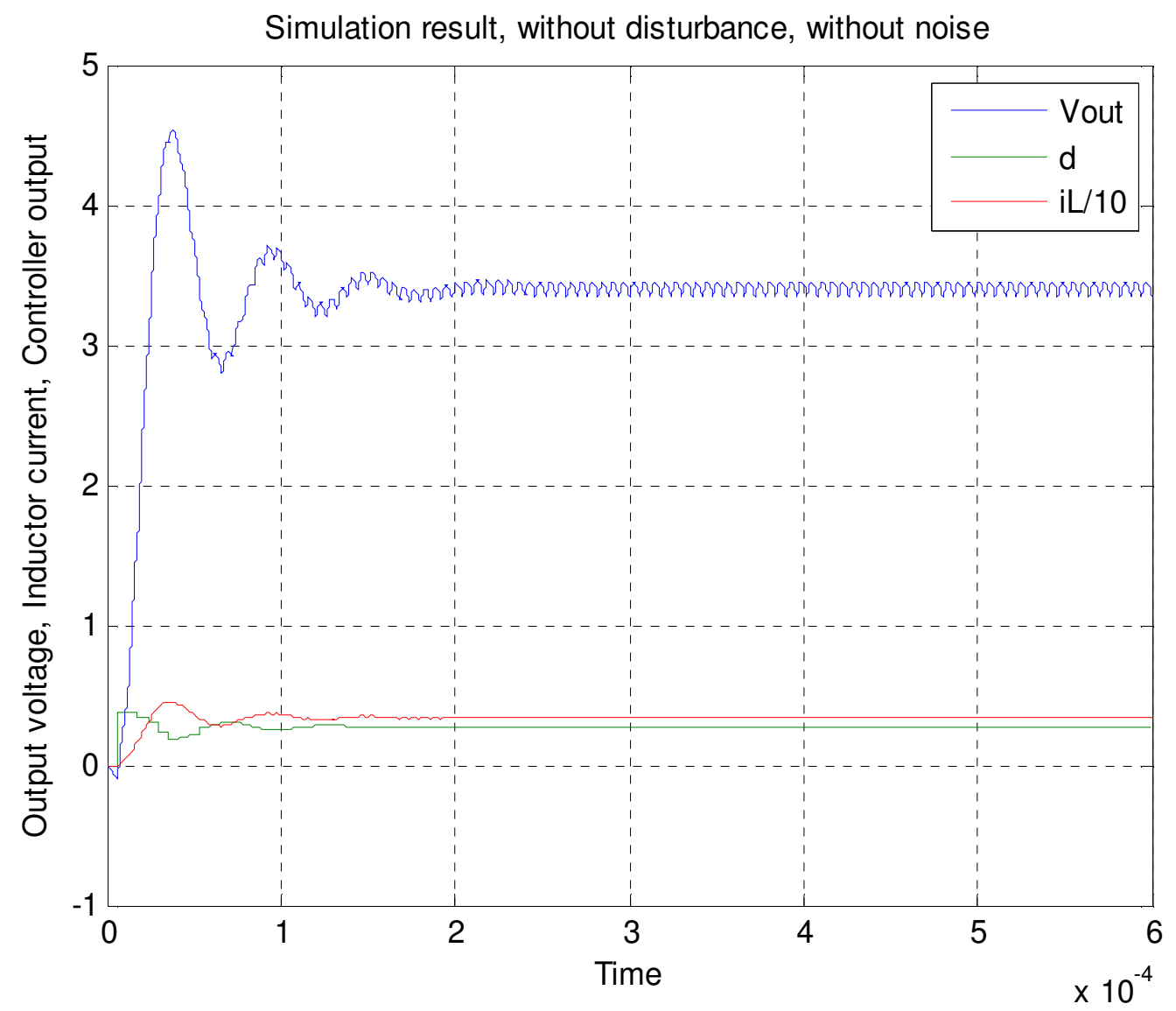

Figure 20: Simulation result, without disturbance and noise.

As it can be seen from above figure 20, with large resistors results in bad simulation result. The output voltage did not reach the stable reference voltage $3.3 \mathrm{~V}$, and it can be observed by viewing the control signal $d$, that the system is not stable.

By modifying the controller design the system could be stable. The modified design implies that the closed system gets a lower bandwidth. 


\section{Chapter 5. PID Controller:}

Here the PID stands for P -Proportional, I - Integral, D - Derivative. A mathematical function described by PID is applied to the error signal.

$$
\text { Where } \quad V_{\text {error }}=V_{\text {set }}-V_{\text {sensor }}
$$

Here $V_{\text {sensor }}$ defines where you are and $V_{\text {set }}$ represents the difference between where you want to go. Very simple, but yet so powerful, If controller is tuned correctly the signal $V_{\text {sensor }}$ will move closer to $V_{\text {set }}$.

Tuning a system means adjusting three variables $\mathrm{Kp}, \mathrm{Ki}$ and $\mathrm{Kd}$ adding in various amounts of these functions to get the system to behave the way you want too.

\subsection{Transfer Function of PID:}

Consider the continuous-time PID Controller transfer function [9] as shown below,

$$
H_{P I D}(s)=K\left[1+\frac{1}{T_{i} s}+\frac{T_{d} s}{1+\frac{T_{d}}{N} s}\right]
$$

This controller is designed by four tuning parameters:

- $K$ - proportional gain

- $T i$ - integral action

- $T d$ - derivative action

- $T d / N-$ filtering of the derivative action

Various discretization methods may be applied to design the structure of the digital PID Controller. The relation among the parameters of the continuous-time and discrete-time will completely depend on the method used, but the structure remains the same for the digital PID Controller [8].

In our case the controller's design and implementation is in the discrete-time, the method of discretization is not crucial. We used the backward difference approximation. It follows that derivative $s$ will be approximated by $\left(1-z^{-1}\right) / T_{s}$ and $1 / s$ [integration] will be approximated by $T_{s} /\left(1-z^{-1}\right)$ [9]. As it is a real time system the possible way is to approximate it with some kind of backward approximation. So, we used backward difference approximation for the system. 
This produces

$$
\begin{gathered}
\frac{1}{T_{i} s}=\frac{T_{s}}{T_{i}} \cdot \frac{1}{1-z^{-1}} \\
T_{d} s=\frac{T_{d}}{T_{s}} \cdot\left(1-z^{-1}\right) \\
\frac{1}{1+\frac{T_{d}}{N} s}=\frac{1}{1+\frac{T_{d}}{N T_{s}}\left(1-z^{-1}\right)}=\frac{\frac{N T_{s}}{T_{d}+N T_{s}}}{1-\frac{T_{d}}{T_{d}+N T_{s}} z^{-1}}
\end{gathered}
$$

By introducing the above expressions in Equation 38, the pulse transfer function [Operator] of the digital PID Controller is obtained:

$$
H_{P I D}\left(z^{-1}\right)=\frac{R\left(z^{-1}\right)}{S\left(z^{-1}\right)}=K\left[1+\frac{T_{s}}{T_{i}} \cdot \frac{1}{1-z^{-1}}+\frac{\frac{N T_{s}}{T_{d}+N T_{s}} \cdot\left(1-z^{-1}\right)}{1-\frac{T_{d}}{T_{d}+N T_{s}} z^{-1}}\right]
$$

Here $\mathrm{R}\left(\mathrm{z}^{-1}\right)$ and $\mathrm{S}\left(\mathrm{z}^{-1}\right)$ are same similar as that of $\mathrm{C}$ and $\mathrm{D}$ notation of the parameters in polynomial controller.

The ratio of two polynomials expression is obtained by adding the three terms. Polynomials $R$ and $S$ have the following form,

$$
\begin{gathered}
R\left(z^{-1}\right)=\left(r 0+r 1 z^{-1}+r 2 z^{-2}\right) \\
S\left(z^{-1}\right)=\left(1+s 1 z^{-1}+s 2 z^{-2}\right)
\end{gathered}
$$

Finding parameters $r_{0}, r_{1}, r_{2}, s_{1}$ and $s_{2}$.

For simplicity consider $G=\frac{T_{d}}{T_{d}+N T_{s}}$ and substitute in equation 40 
Then equation becomes $K\left[1+\frac{T_{s}}{T_{i}}\left(\frac{1}{1-z^{-1}}\right)+\frac{\frac{G N T_{s}}{T_{d}}\left(1-z^{-1}\right)}{1-G \cdot z^{-1}}\right]$

$$
=\frac{K\left[\left(1-G \cdot z^{-1}\right)\left(T_{i}-T_{i} \cdot z^{-1}\right)+\frac{G T_{s}\left(1-z^{-1}\right) T_{i}\left(1-z^{-1}\right)+T_{s}\left(1-G \cdot z^{-1}\right)}{T_{d}}\right]}{T_{i}\left(1-z^{-1}\right)\left(1-G \cdot z^{-1}\right)}
$$

Now consider Denominator Part $T_{i}\left(1-z^{-1}\right)\left(1-G \cdot z^{-1}\right)$

Now Substitute $G=\frac{T_{d}}{T_{d}+N T_{s}}$

$$
\begin{aligned}
& T_{i}\left(1-z^{-1}\right)\left(1-G \cdot z^{-1}\right)=T_{i}\left(1-z^{-1}\right)\left(1-\frac{T_{d}}{T_{d}+N T_{s}} \cdot z^{-1}\right) \\
& =T_{i}-\frac{T_{i} T_{d}}{T_{d}+N T_{s}} z^{-1}-z^{-1} T_{i}+\frac{T_{d} T_{i}}{T_{d}+N T_{s}} z^{-2} \\
& =T_{i}\left[1-\frac{T_{d}}{T_{d}+N T_{s}} z^{-1}-z^{-1}+\frac{T_{d}}{T_{d}+N T_{s}} z^{-2}\right]
\end{aligned}
$$

$T_{i}$ Cancelled in both numerator and denominator

$$
\begin{aligned}
& =1-z^{-1}\left[\frac{-2 T_{d}+N T_{s}}{T_{d}+N T_{s}}\right]+\frac{T_{d}}{T_{d}+N T_{s}} z^{-2} \\
& =1+z^{-1}\left[\frac{-2 T_{d}+N T_{s}}{T_{d}+N T_{s}}\right]+\frac{T_{d}}{T_{d}+N T_{s}} z^{-2}=1+s_{1} z^{-1}+s_{2} z^{-2}=\left(1-z^{-1}\right)\left(1+s_{1}^{\prime} z^{-1}\right)
\end{aligned}
$$

Now $s_{1}^{\prime}=-s_{2}=-\frac{T_{d}}{T_{d}+N T_{s}}$ and $s_{1}=\left[\frac{-2 T_{d}+N T_{s}}{T_{d}+N T_{s}}\right]$ 
Now for simplicity again take $G=\frac{T_{d}}{T_{d}+N T_{s}}$

Now take numerator part from equation $\mathbf{4 2}$ i.e.

$$
\begin{aligned}
& K\left[\left(1-G \cdot z^{-1}\right)\left(T_{i}-T_{i} \cdot z^{-1}\right)+\frac{G T_{s}\left(1-z^{-1}\right) T_{i}\left(1-z^{-1}\right)+T_{s}\left(1-G \cdot z^{-1}\right)}{T_{d}}\right] \\
& K\left[T_{i}-T_{i} z^{-1}-G T_{i} z^{-1}+G T_{i} z^{-2}+T_{s}-G T_{s} z^{-1}+\frac{G N T_{s} T_{i}}{T_{d}}-\frac{2 G N T_{s} T_{i} z^{-1}}{T_{d}}-\frac{G N T_{s} T_{i} z^{-2}}{T_{d}}\right]
\end{aligned}
$$

Now taking $z^{-1}$ and $z^{-2}$ as common in above equation

$$
K\left[z^{-1}\left[-T_{i}-G T_{i}-G T_{s}-\frac{2 G N T_{s} T_{i}}{T_{d}}\right]+z^{-2}\left[G T_{i}-\frac{G N T_{s} T_{i}}{T_{d}}\right]+\left[T_{i}+T_{s}+\frac{G N T_{s} T_{i}}{T_{d}}\right]\right]
$$

Now taking $T_{i}$ as common in above equation

$$
T_{i} K\left[z^{-1}\left[-1-G-\frac{G T_{s}}{T_{i}}-\frac{2 G N T_{s}}{T_{d}}\right]+z^{-2}\left[G-\frac{G N T_{s}}{T_{d}}\right]+\left[1+\frac{T_{s}}{T_{i}}+\frac{G N T_{s}}{T_{d}}\right]\right]
$$

Now Substitute $G=\frac{T_{d}}{T_{d}+N T_{s}}$

$$
\begin{aligned}
& K\left[1+\frac{T_{s}}{T_{i}}+\frac{T_{d}}{T_{d}+N T_{s}} \cdot \frac{N T_{s}}{T_{d}}\right]+K \cdot z^{-1}\left[-1-\frac{T_{d}}{T_{d}+N T_{s}}-\frac{T_{d}}{T_{d}+N T_{s}} \cdot \frac{T_{s}}{T_{i}}-2 \frac{T_{d}}{T_{d}+N T_{s}} \cdot \frac{N T_{s}}{T_{d}}\right]+ \\
& K . z^{-2}\left[\frac{T_{d}}{T_{d}+N T_{s}}-\frac{T_{d}}{T_{d}+N T_{s}} \cdot \frac{N T_{s}}{T_{d}}\right]=r_{0}+r_{1} z^{-1}+r_{2} z^{-2}
\end{aligned}
$$

We know that $s_{1}^{\prime}=-\frac{T_{d}}{T_{d}+N T_{s}}$ and substitute in 43 equation. 


$$
K\left[\begin{array}{l}
{\left[\begin{array}{l}
\left.1+\frac{T_{s}}{T_{i}}-s_{1}^{\prime} \cdot \frac{N T_{s}}{T_{d}}\right] \\
z^{-2}\left[-s_{1}^{\prime}+s_{1}^{\prime} \cdot \frac{N T_{s}}{T_{d}}\right]
\end{array}\right] z^{-1}\left[-1+s_{1}^{\prime}+\frac{T_{s}}{T_{i}} s_{1}^{\prime}+2 s_{1}^{\prime} \cdot \frac{N T_{s}}{T_{d}}\right]+}
\end{array}\right]=r_{0}+r_{1} z^{-1}+r_{2} z^{-2}
$$

From equation 44 and 47 we get

$$
\begin{aligned}
& r_{0}=K\left[1+\frac{T_{s}}{T_{i}}+\frac{T_{d}}{T_{d}+N T_{s}} \cdot \frac{N T_{s}}{T_{d}}\right] \\
& r_{1}=K\left[-1-\frac{T_{d}}{T_{d}+N T_{s}}\left[1+\frac{T_{s}}{T_{i}}+2 \cdot \frac{N T_{s}}{T_{d}}\right]\right] \\
& r_{2}=K\left[-s_{1}^{\prime}+s_{1}^{\prime} \cdot \frac{N T_{s}}{T_{d}}\right]=K \frac{T_{d}}{T_{d}+N T_{s}}\left[1+\frac{N T_{s}}{T_{d}}\right] \\
& s 1=-\frac{2 T_{d}+N T_{s}}{T_{d}+N T_{s}} \\
& s 2=\frac{T_{d}}{T_{d}+N T_{s}}
\end{aligned}
$$

From the parameters $r_{0}, r_{1}, r_{2}, s_{1}$ and $s_{2}$ we get $K, T_{i}, T_{d}$ and $\frac{T_{d}}{N}$.

$$
\begin{aligned}
& K=\frac{r_{0}\left[-\frac{T_{d}}{T_{d}+N T_{s}}\right]-r_{1}-\left(2-\frac{T_{d}}{T_{d}+N T_{s}}\right) r_{2}}{\left(1+-\frac{T_{d}}{T_{d}+N T_{s}}\right)^{2}} \\
& T_{i}=T_{s} \cdot \frac{K\left(1-\frac{T_{d}}{T_{d}+N T_{s}}\right)}{r_{0}+r_{1}+r_{2}}
\end{aligned}
$$


$T_{d}=T_{s} \cdot \frac{\left[-\frac{T_{d}}{T_{d}+N T_{s}}\right] r_{0}+\frac{T_{d}}{T_{d}+N T_{s}}+r_{2}}{K\left[1-\frac{T_{d}}{T_{d}+N T_{s}}\right]^{3}}$

$$
\frac{T_{d}}{N}=\frac{\left[-\frac{T_{d}}{T_{d}+N T_{s}}\right] T_{s}}{1-\frac{T_{d}}{T_{d}+N T_{s}}}
$$

\subsection{Structure of the Digital PID Controller:}

The digital PID Controller has five parameters $[r 0, r 1, r 2, s 1, s 2]$ as the continuous time PID Controller.

The pulse transfer function [operator] of the digital PID Controller contains as a common factor of the denominator the term $\left(1-z^{-1}\right)$, it assures the behavior of the numerical integration. The denominator also contains the term $\left(1-s 1 z^{-1}\right)$, this is a digital filter which plays the role of the filter $[1+(T d / N) s]$ in the continuous-time PID Controller.

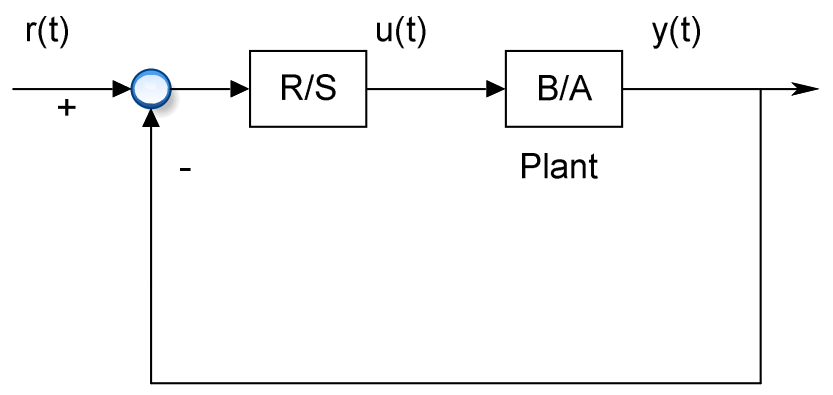

Figure 21: Equivalent block diagrams of a digital PID Controller.

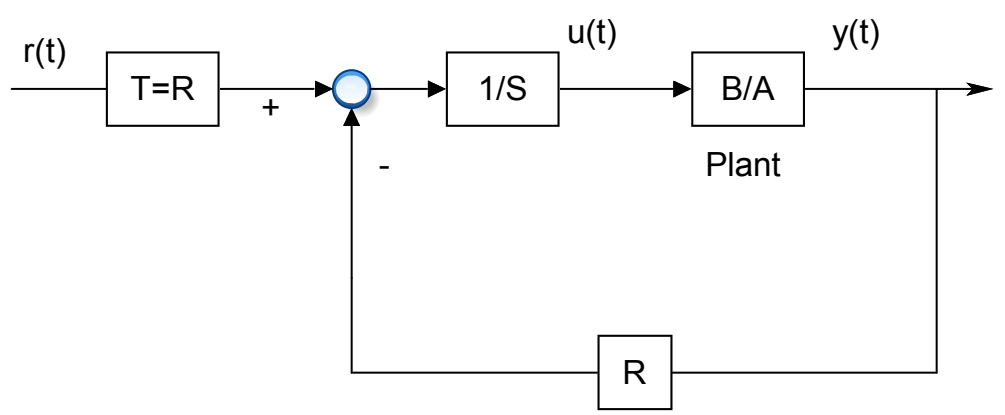

Figure 22: Equivalent block diagrams of a digital control loop using the digital PID Controller. 
Computation of the coefficients of the Digital Controller:

$$
H(z)=\frac{B(z)}{A(z)}=\frac{b 1 z^{2}+b 2 z+b 3}{z^{3}+a 1 z^{2}+a 2 z+a 3}
$$

$$
P\left(z^{-1}\right)=A\left(z^{-1}\right) S\left(z^{-1}\right)+B\left(z^{-1}\right) R\left(z^{-1}\right)
$$

$R\left(z^{-1}\right)=\left(r_{0}+r_{1} z^{-1}+r_{2} z^{-2}\right)$

$S\left(z^{-1}\right)=\left(1+s_{1} z^{-1}+s_{2} z^{-2}\right)$

$P\left(z^{-1}\right)=A\left(z^{-1}\right) S\left(z^{-1}\right)+B\left(z^{-1}\right) R\left(z^{-1}\right)$

$=\left(1+a 1 z^{-1}+a 2 z^{-2}+a 3 z^{-3}\right)\left(1+s_{1} z^{-1}+s_{2} z^{-2}\right)+\left(b 1 z^{-1}+b 2 z^{-2}+z^{-3}\right)\left(r_{0}+r_{1} z^{-1}+r_{2} z^{-2}\right)$

The remaining equation is in Appendix 4.

The values for poles q1, q2, q3, q4 andq5 have been chosen as $0.5,0.5,0.5,0.5$ and 0.5 respectively.

Here we had taken 0.5 for the poles because, we had tried different combinations of the poles with frequency for both the PID and Polynomial controllers i.e.

The resulted graphs are seen in the Appendix-5.

At $1 \mathrm{e}^{-6}$, the following pole combination are consider

$q 1=0.1, q 2=0.2, q 3=0.3, q 4=0.4, q 5=0.5$

$q 1=0.3, q 2=0.4, q 3=0.5, q 4=0.6, q 5=0.7$ and

$q 1=0.5, q 2=0.5, q 3=0.5, q 4=0.5, q 5=0.5$

At this output $1 \mathrm{e}^{-6}$, and at poles $\mathrm{q} 1=0.5, \mathrm{q} 2=0.5, \mathrm{q} 3=0.5, \mathrm{q} 4=0.5, \mathrm{q} 5=0.5$, the result is better than the remaining combinations, so we consider these pole values for both the controllers. And the same formula is used as in equation 26. 


\subsection{Simulation results of the Continuous and Discrete model:}

The below graph Figure 23, is the output of the discrete model

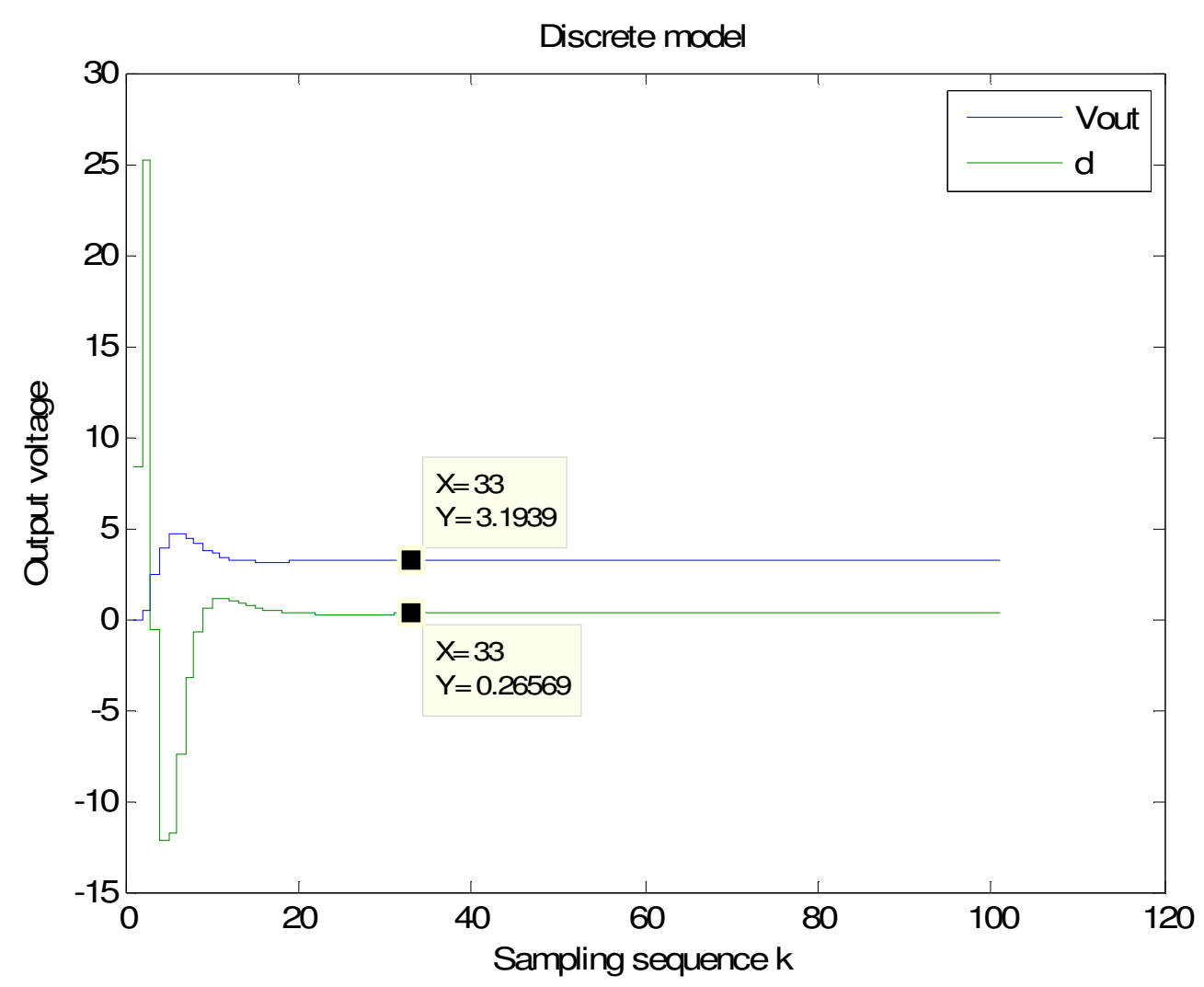

Figure 23: Discrete model output

We can observe that, the output voltage of discrete system is little different to the required voltage. Were the reference voltage is 3.3 but the discrete model output is 2.904 , which is 0.3 volts different from reference voltage.

The output of the controller gets stable quickly [in 20 sample intervals] and remains stable. The controller attains the output voltage come to stable status quickly [in 20 sample intervals] and remains the output voltage $2.9 \mathrm{~V}$ [reference voltage $3.3 \mathrm{~V}$ ].

The simulation is based on Digital PID Controller theory; it is a representation of the theory by numerical calculations. While designing the controller, the saturation limits should not be reached too much for a reasonable disturbance. And the simulation result satisfies it.

The Discrete time Simulink model without saturation for PID Controller, shown below. 


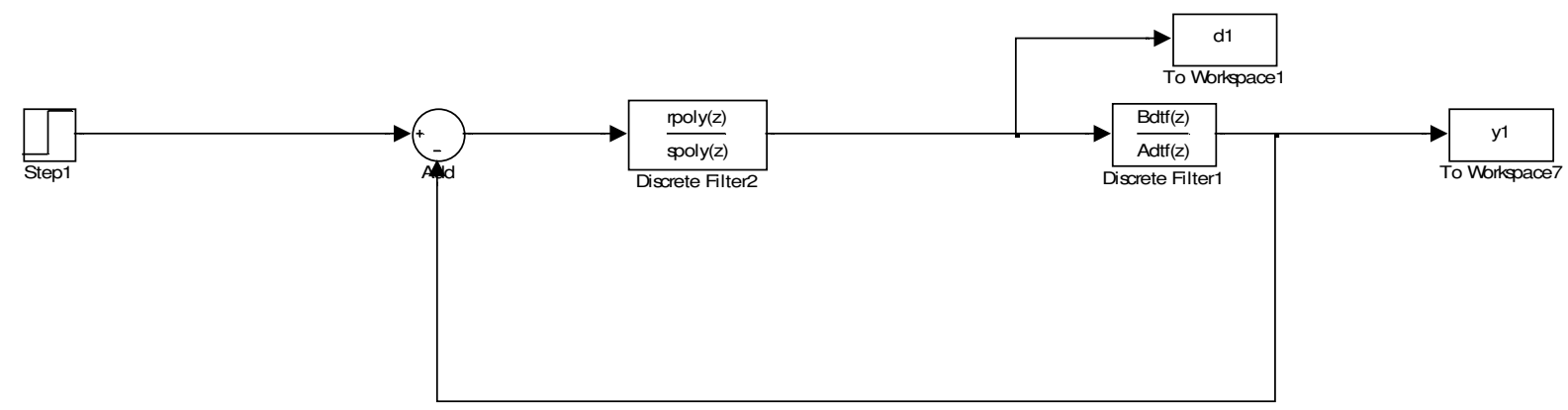

Figure 24: Discrete Time Model without Saturation.

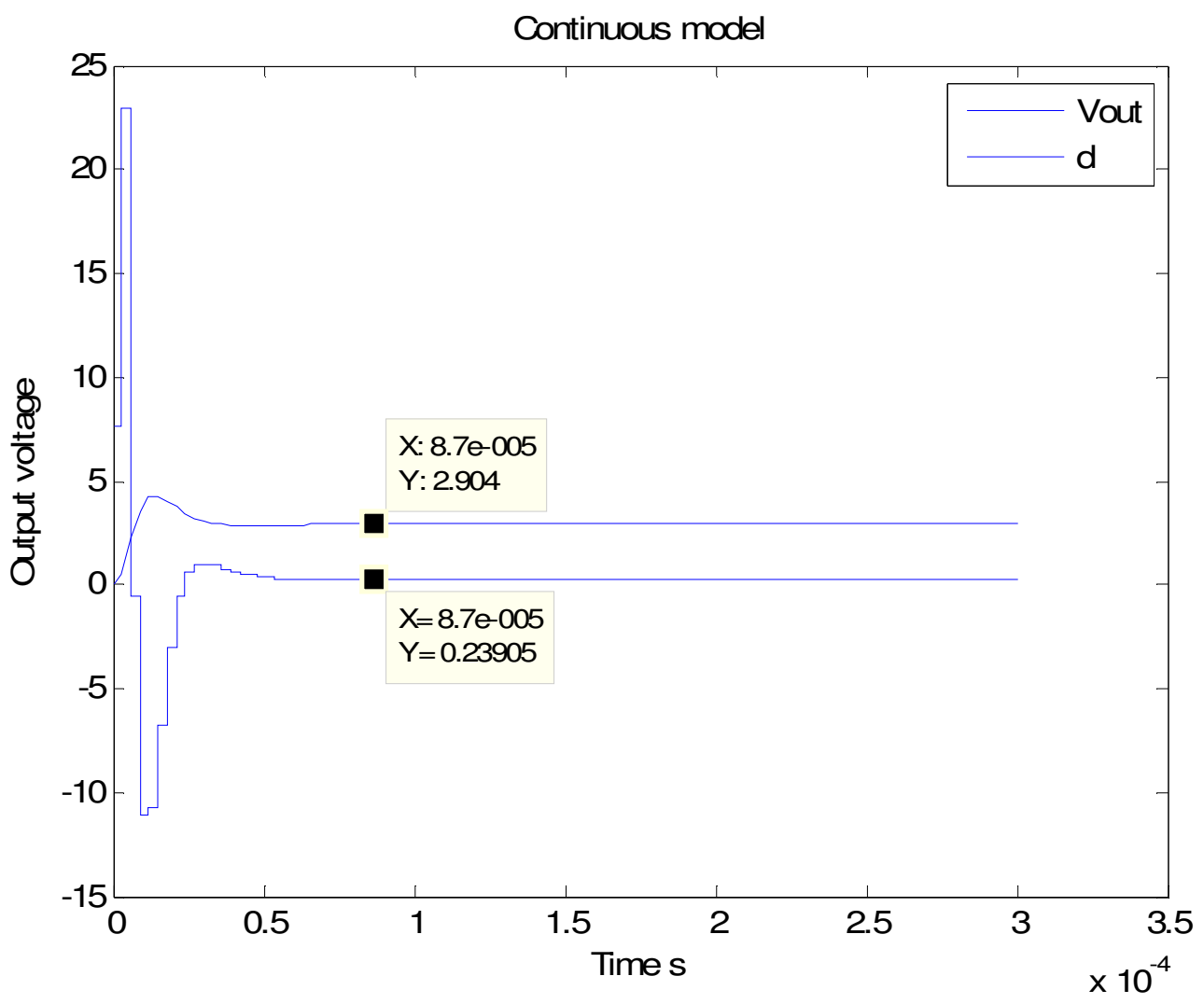

Figure 25: Continuous output

Since the reference voltage is $3.3 \mathrm{~V}$, the output voltage did not reach it very well. We can observe that the output voltage remains stable at nearly at $3 \mathrm{~V}$. 
The controller attains the output voltage to be stable quickly [in $50 \mu \mathrm{s}$ ] and remains stable below the reference voltage [3.3V].

The Continuous time Simulink model without saturation for PID Controller, shown below.

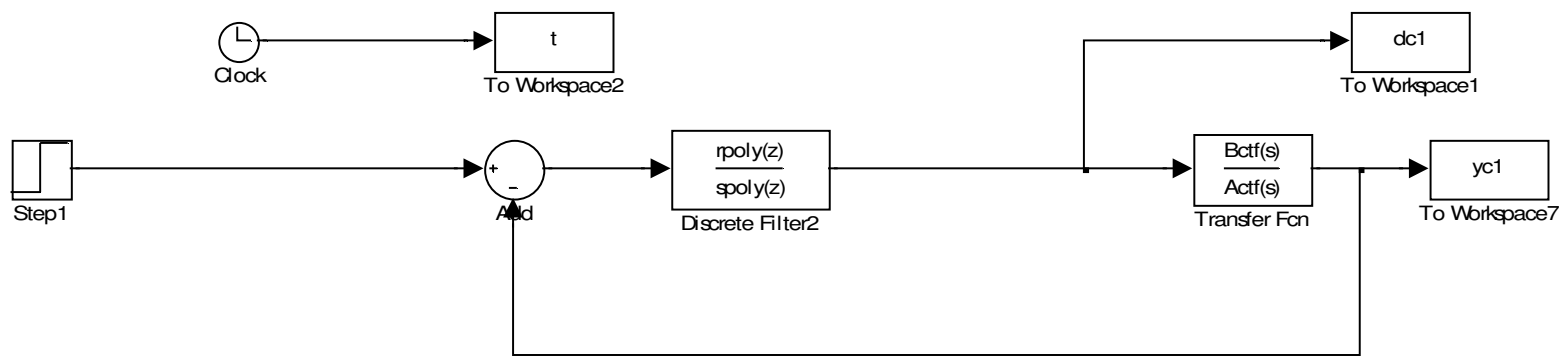

Figure 26: Continuous Time Model without Saturation.

\subsection{Simulation result without disturbance and without noise:}

The model is a switched time continuous model. From these results we can tell that how good the controller works for this system with disturbance.

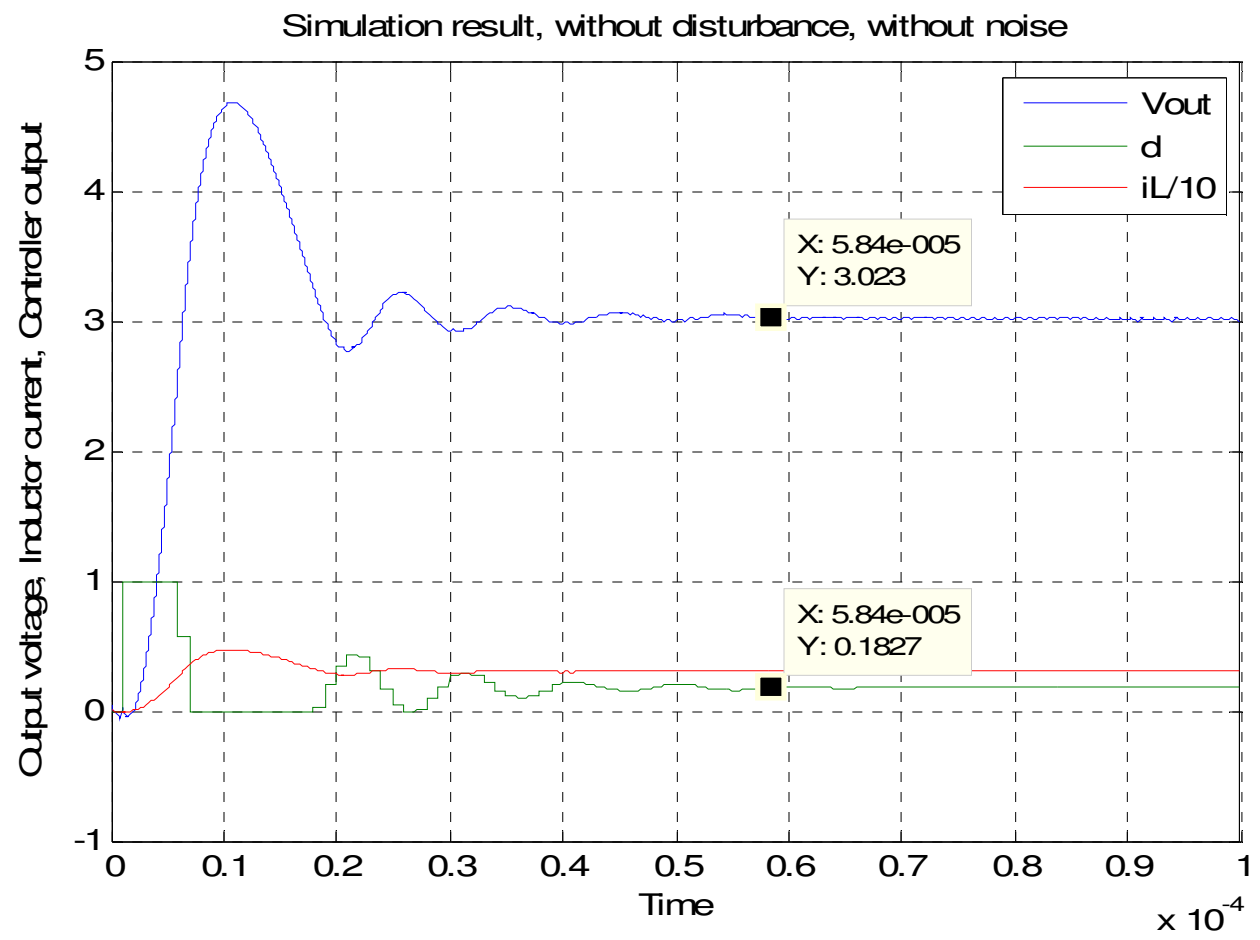

Figure 27: Simulation output for without disturbance and without noise. 
It can be observable from the above output figure, the output voltage [3v] is quite stable and not so nearer to the given reference voltage i.e. $3.3 \mathrm{v}$. And the controller output is also very stable near $0.182=3.023 / 12$ [reference voltage divide input voltage].

The block diagram of PWM model with controller Simulink is shown below.

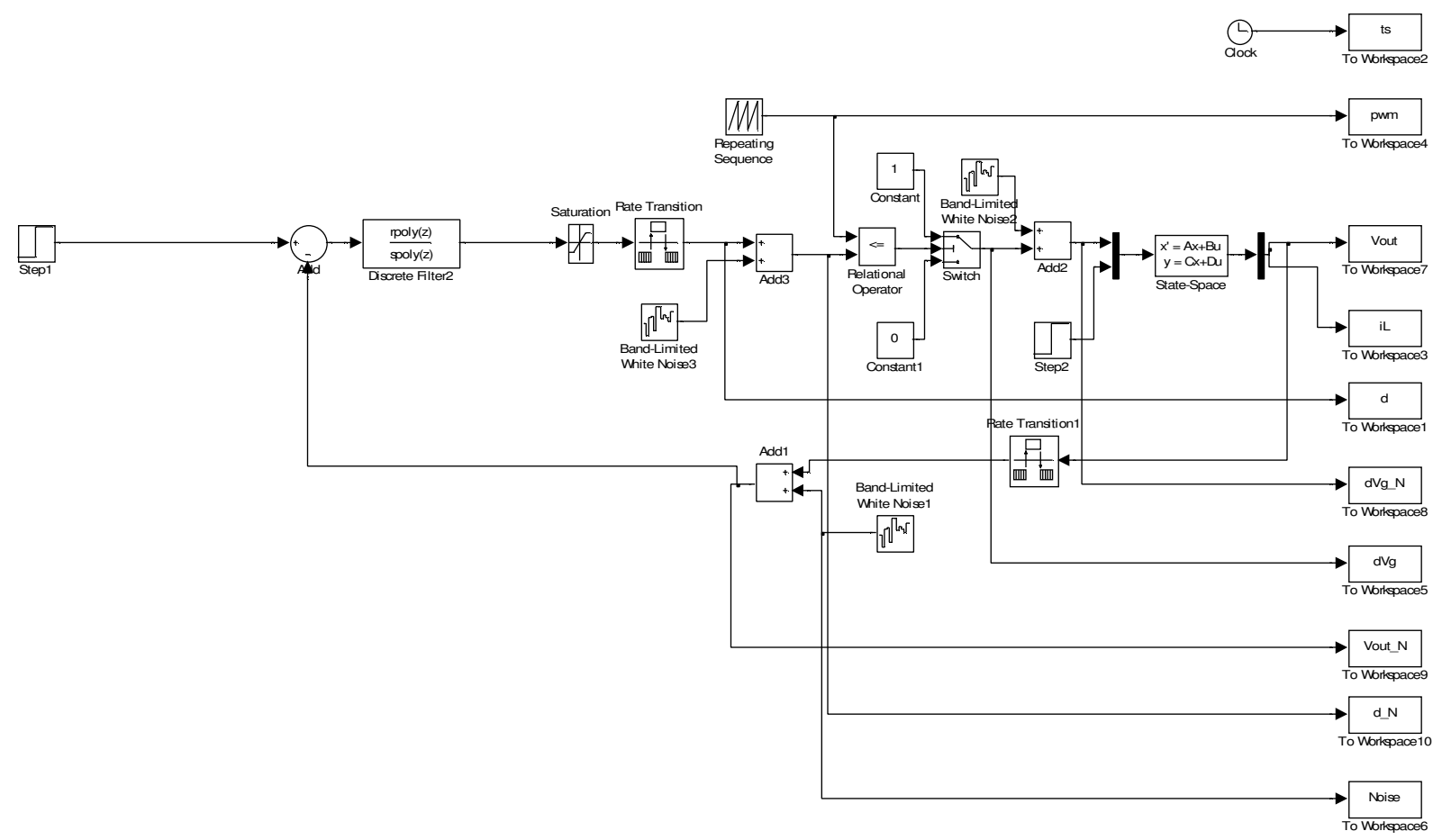

Figure 28: PWM model with controller Simulink

\subsection{Simulation result without Disturbance and with Noise:}

In the realistic case, the circuit has little noise, from the switch with respect to high frequency switching, measurement noise and so on. Here, we add some band-limited white noise in simulation.

The noise frequency is $33 \mathrm{MHz}$ and the noise magnitude is between -0.2 and 0.2 , here frequency is a approximate estimation based on measurement; see Figure 30 and the measurements are introduced by the switching, but use it the whole time. 


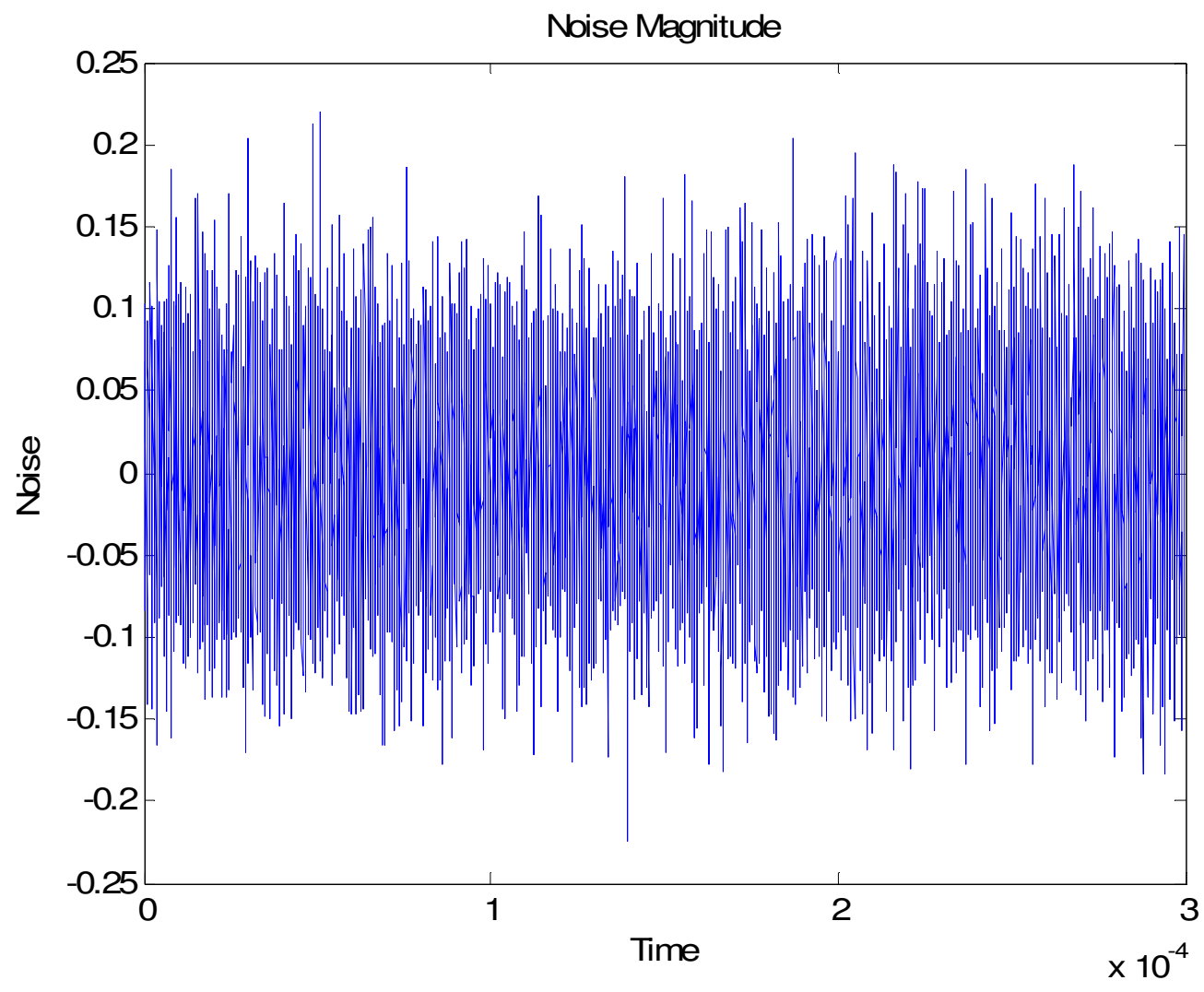

Figure 29: Simulation output for without disturbance with noise

The noise applied in this simulation is very suitable, and it is moderate i.e., nor small or big. So here we used the same form of noise in the simulation.

The noise is sum up with the output voltage as the measurement noise and the output voltage with noise can be seen in Figure 30. 


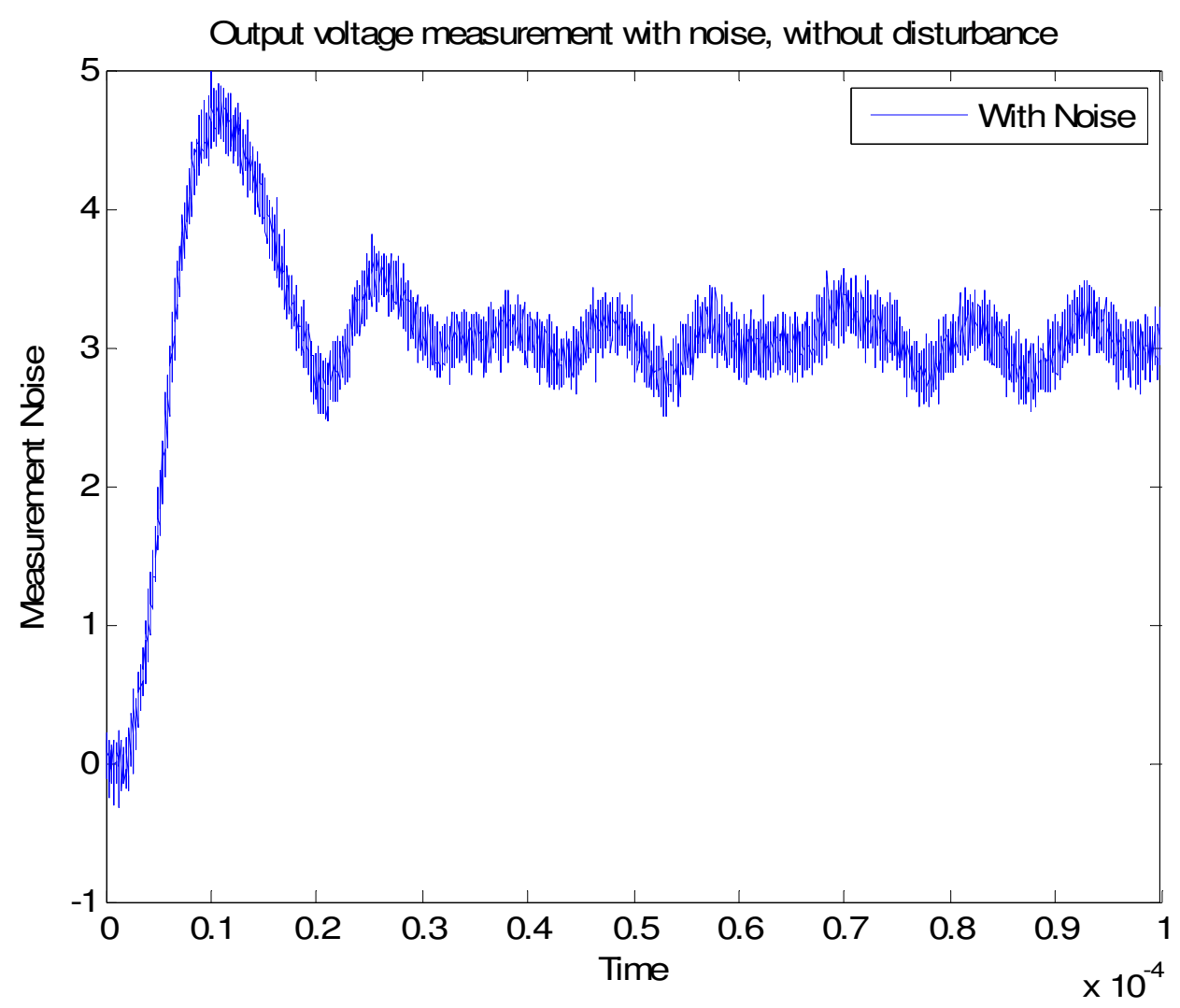

Figure 30: simulation output for voltage measurement with noise and without disturbance.

It can be observable after adding some amount of noise, the output is not stable, but the noise is not huge it has effect on the stability of controller. When the output couldn't get stable [range is $3.0 \mathrm{~V}-3.5 \mathrm{~V}$ ] as the result, the controller with noise is less stable when compared to the controller without noise.

\subsection{Simulation result with Disturbance and without Noise:}

In this model, the current source is applied as the load, when the output current abruptly changes; there is some sort of disturbance in the output voltage.

To notice, the disturbance and check whether the PID Controller has ability to turn back the output voltage to reference voltage. Set the current change range as $5 A-15 A$ see in Figure 30. 


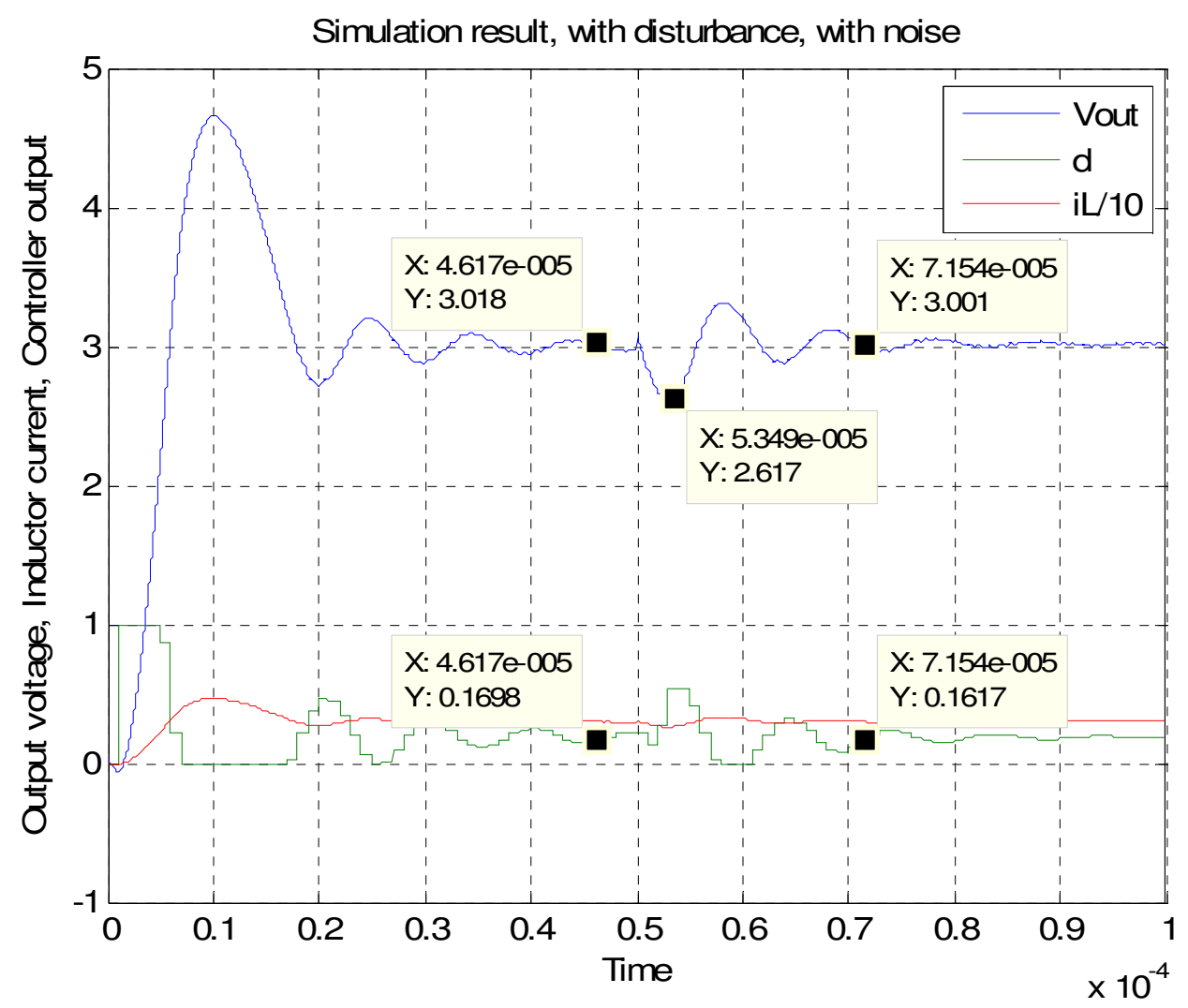

Figure 31: Simulation output with disturbance and noise

The output voltage under control is about $3.018 \mathrm{~V}$ it is not quite close to the reference voltage $3.3 \mathrm{~V}$; it means the controller is not good enough. Then changing the current at the load from $5 \mathrm{~A}$ to $15 \mathrm{~A}$, the output voltage falls from $3.018 \mathrm{~V}$ to $2.617 \mathrm{~V}$.

It recover to the stable voltage $3.001 \mathrm{~V}$ shortly [in 10 sample intervals] when the load current changes abruptly, the controller output voltage recovers to the stable voltage soon.

\subsection{Simulation result with Disturbance and Noise:}

Adding the same noise like, what was done in section 5.6 and for the simulation results we can refer to Figure 32. 


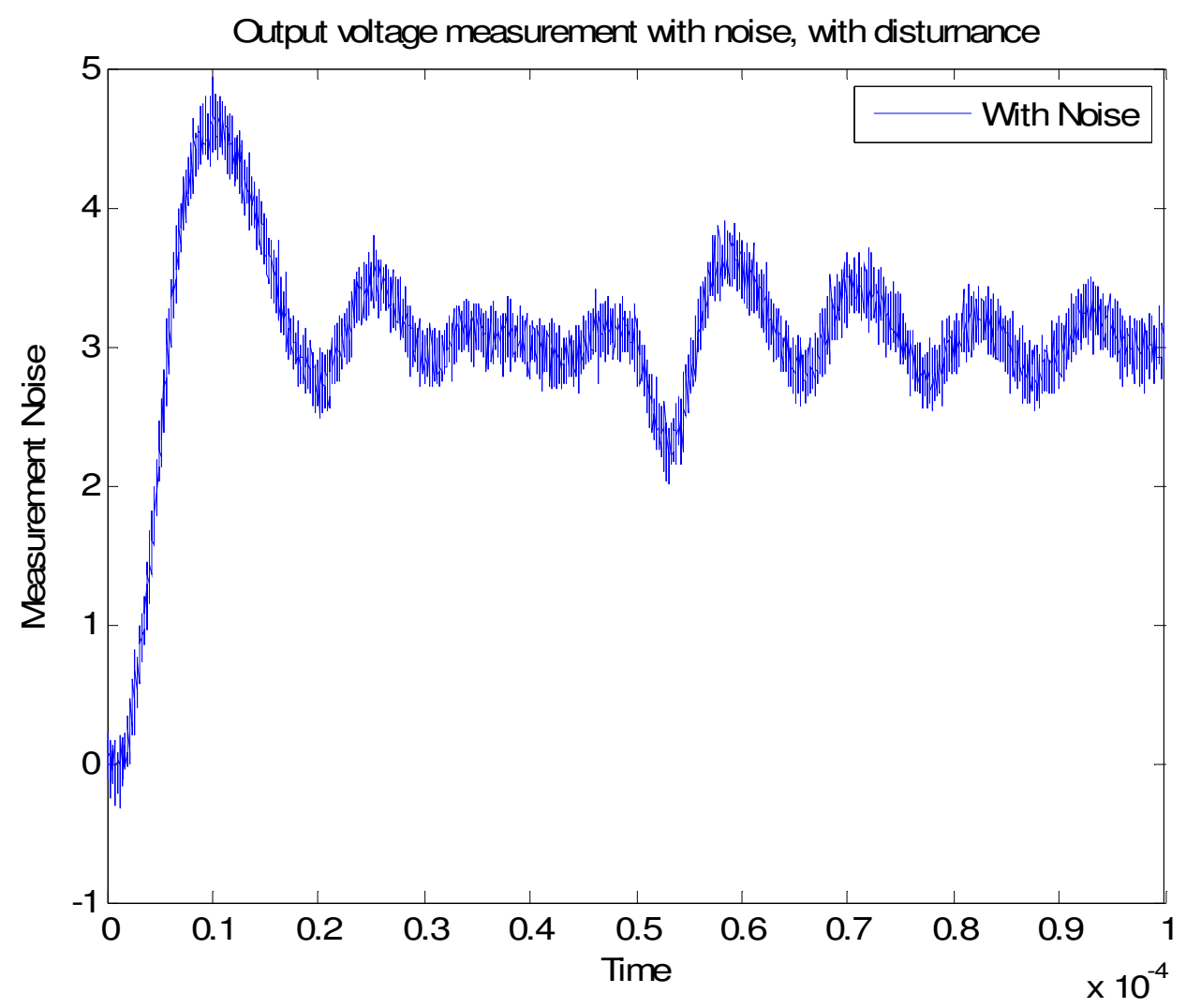

Figure 32: Simulation output with disturbance and noise

As it can be observable, after summing the noise, the output voltage is not as stable as before. The output voltage is not closer to the reference voltage and the controller output is close to 0.280 but not so stable.

The simulation need to try large resistors, and observe the controller quality. For the simulation result, see Figure 33. 


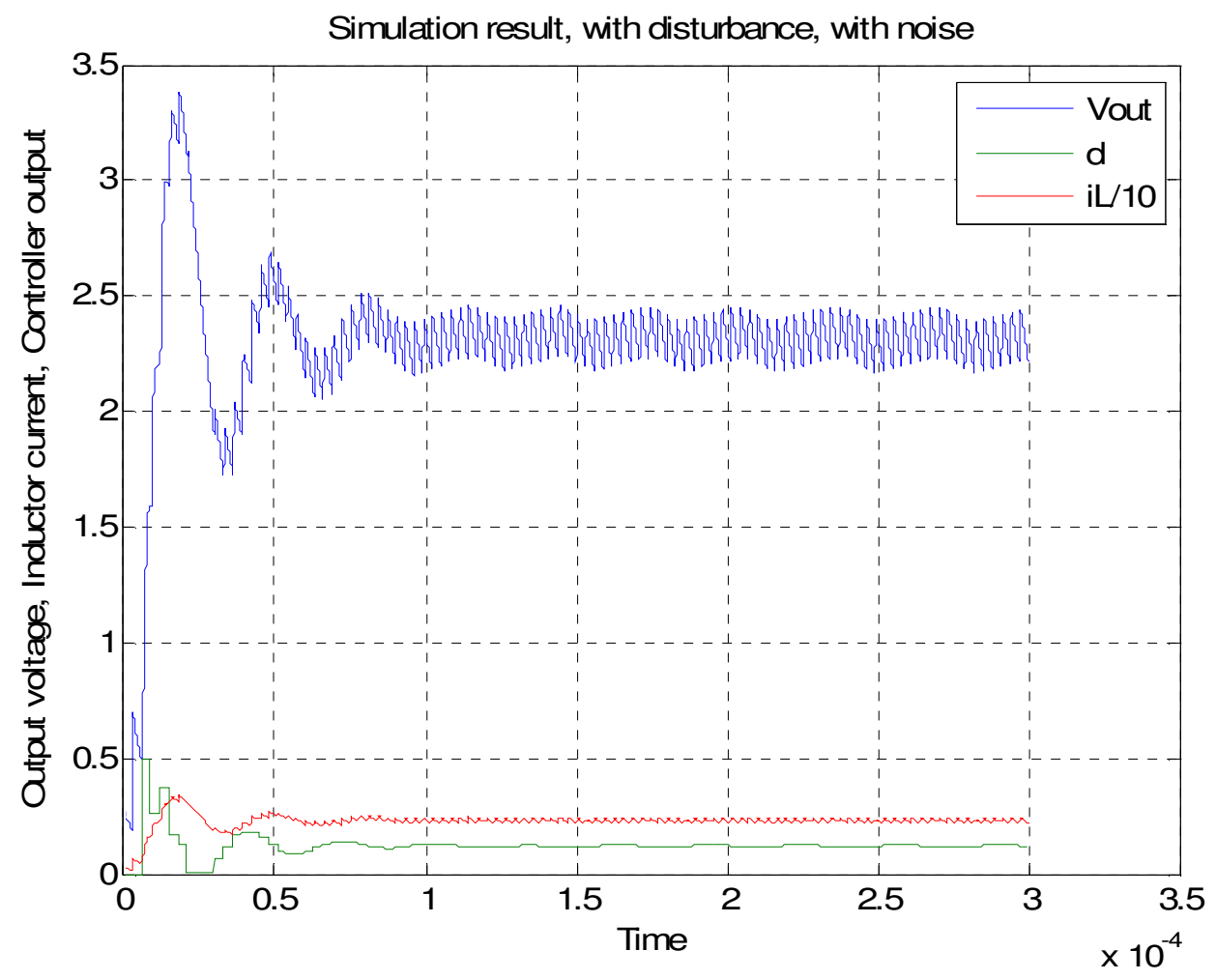

Figure 33: Simulation output with disturbance and noise, with large resistance.

As it can be observable, after summing the noise, the figure 33 shows the output voltage with oscillations. When compared with the polynomial controller the PID did not reached the given reference voltage $3.3 \mathrm{v}$. While the polynomial controller performed well with the reference voltage. 


\section{Chapter 6. PD Controller:}

In this Controller describes three basic mathematical functions applied to the error signal, $V_{\text {error }}=V_{\text {set }}-V_{\text {sensor }}$ here the controller performs the PD mathematical functions on the error and applies their sum to the system.

Proportional-Derivative control is useful for fast response controllers, here we had discussed about the two controller actions.

\section{Proportional Action:}

Proportional action gives a quick response to the control error. This is useful for improving the response of a stable system. Additionally, the gain is the same for all frequencies leaving the system with a none zero steady-state error.

\section{Derivative Action:}

Derivative action acts on the rate of change of the control error. This provides a fast response, as opposed to the integral action, it will produce large control signals in response to high frequency control errors such as set point changes [step command] and measurement noise.

\subsection{Structure of the digital PD controller:}

The digital PD Controller has five parameters [d0, $d 1, \& c 1]$ as the continuous time PD Controller.

The pulse transfer function [operator] of the digital PID Controller contains as a common factor of the denominator the term $\left(1-z^{-1}\right)$, it assures the behavior of the numerical integration. The denominator also contains the term $\left(1-s 1 z^{-1}\right)$, this is a digital filter which plays the role of the filter $[1+(T d / N) s]$ in the continuous-time PD Controller. 


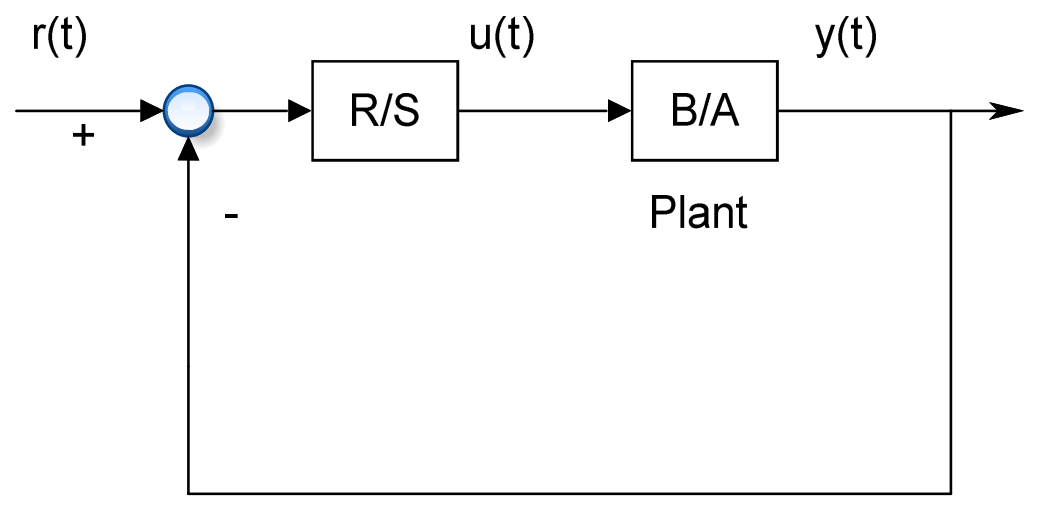

Figure 34: Equivalent block diagrams of a digital PD Controller.

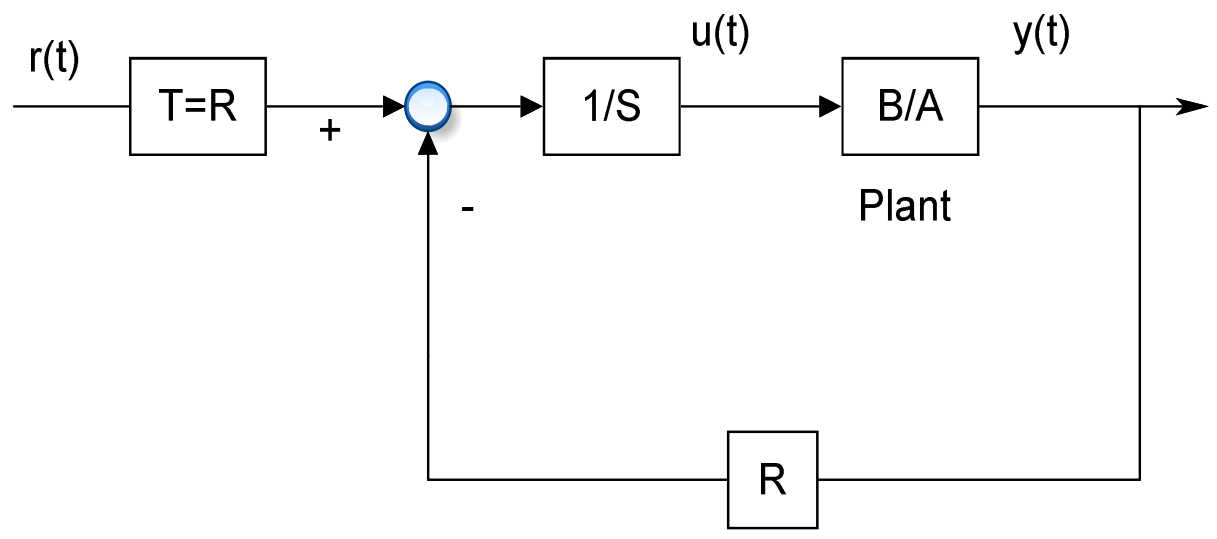

Figure 35: Equivalent block diagrams of a digital control loop using the digital PD Controller.

Here in this thesis we considering a second order PD Controller plant, with the transfer function obtained from the plant

$$
\left(\frac{k\left(1+T_{d} s\right)}{1+\frac{T_{d}}{N} s}\right)
$$

And discredited according to Tustin method. 


$$
s=\left(\frac{2}{h} \frac{1-z^{-1}}{1+z^{-1}}\right)
$$

Substituting s value in equation 66,

$$
\begin{aligned}
& \mathrm{K} \frac{1+T_{d} \frac{2}{h} \frac{1-z^{-1}}{1+z^{-1}}}{1+\frac{T_{d}}{N} \frac{2}{h} \frac{1-z^{-1}}{1+z^{-1}}} \\
& \mathrm{~K} \frac{1+z^{-1}+T_{d} \frac{2}{h}\left(1-z^{-1}\right)}{1+z^{-1}+\frac{T_{d}}{N} \frac{2}{h}\left(1-z^{-1}\right)} \\
& \mathrm{K} \frac{\left(1+T_{d} \frac{2}{h}\right)+\left(1-T_{d} \frac{2}{h}\right) z^{-1}}{\left(1+\frac{T_{d}}{N} \frac{2}{h}\right)+\left(1-\frac{T_{d}}{N} \frac{2}{h}\right) z^{-1}} \\
& u(k)=c 1 u(k-1)-d 0 y(k)-d 1 y(k-1) \\
& u(z)\left(1+c 1 z^{-1}\right)=y(z)\left(d 0+d 1 z^{-1}\right)
\end{aligned}
$$

From the above equation, the parameters are $c 1, d 0$, and $d 1$ which are used as a part in matlab tool for obtaining the poles for this second order PD controller system.

\section{2 calculations for finding the poles of PD controller of Second order:}

Computation of the coefficients of the Digital Controller:

$$
\begin{aligned}
& H\left(z^{-1}\right)=\left(\frac{B\left(z^{-1}\right)}{A\left(z^{-1}\right)}\right) \\
& =\left(\frac{b 1 z^{-1}+b 2 z^{-2}}{1+a 1 z^{-1}+a 2 z^{-2}}\right)
\end{aligned}
$$


The above mentioned $\mathrm{B}\left(\mathrm{z}^{-1}\right) \& \mathrm{~A}\left(\mathrm{z}^{-1}\right)$ are the transfer function of the second order PD controller $P\left(z^{-1}\right)=A\left(z^{-1}\right) C\left(z^{-1}\right)+B\left(z^{-1}\right) D\left(z^{-1}\right)$

Equation 67 and 68 are substituted in 69 equation

$$
=\left(1+a 1 z^{-1}+a 2 z^{-2}\right)\left(1+c 1 z^{-1}\right)+\left(b 1 z^{-1}+b 2 z^{-2}\right)\left(d 0+d 1 z^{-1}\right)
$$

$C\left(z^{-1}\right) \& D\left(z^{-1}\right)$ are calculated from the second order PD controller by using Tustin method.

$$
\left(1+c 1 z^{-1}+a 1 z^{-1}+a 1 c 1 z^{-2}+a 2 z^{-2}+a 2 c 1 z^{-3}\right)+\left(b 1 d 0 z^{-1}+b 1 d 1 z^{-2}+b 2 d 0 z^{-2}+b 2 d 1 z^{-3}\right)
$$

In the above equation $z^{-1}, z^{-2}$, and $z^{-3}$ are taken as common,

$$
=1+z^{-1}(c 1+a 1+b 1 d 0)+z^{-2}(a 1 c 1+a 2+b 1 d 1+b 2 d 0)+z^{-3}(a 2 c 1+b 2 d 1)
$$

Finding poles $p 1, p 2$, and $p 3$ in terms of $q 1, q 2$, and $q 3$

$$
\begin{aligned}
& P(z)=\left(1-q 1 z^{-1}\right)\left(1-q 2 z^{-1}\right)\left(1-q 3 z^{-1}\right) \\
& =1+p 1 z^{-1}+p 2 z^{-2}+p 3 z^{-3}
\end{aligned}
$$

Then choose the poles $p 1, p 2$, and $p 3$

$$
\begin{aligned}
& P 1=-q 3-q 2-q 1 \\
& P 2=q 2 q 3+q 1 q 3+q 1 q 2 \\
& P 3=-q 1 q 2 q 3
\end{aligned}
$$

Identifying the coefficients for $\mathrm{p}(\mathrm{z})$ from the equation $\mathbf{7 1 .}$

Where $\mathrm{p} 1=c 1+a 1+b 1 d 0$

$$
\begin{aligned}
& \mathrm{p} 2=a 1 c 1+a 2+b 1 d 1+b 2 d 0 \text { and } \\
& \mathrm{p} 3=a 2 c 1+b 2 d 1
\end{aligned}
$$

From the above obtained $\mathrm{p} 1, \mathrm{p} 2$ and $\mathrm{p} 3 ; \mathrm{d} 0, \mathrm{~d} 1$ and $\mathrm{c} 1$ parameters are extracted.

$\mathrm{d} 0=\left(\frac{p 1-c 1-a 1}{b 1}\right), \mathrm{d} 1=\left(\frac{p 3-a 2 c 1}{b 2}\right)$ and for extracting $\mathrm{c} 1$ parameter $\mathrm{d} 0$ and $\mathrm{d} 1$ values are 
substituted in the $\mathrm{p} 2$ equation, as shown below

$\mathrm{p} 2=a 1 c 1+a 2+b 1 d 1+b 2 d 0$

Simplification is shown in Appendix 6.

$$
c 1=\left(\frac{b 1 b 2(p 2-a 2)+b 2^{2}(a 1-p 1)-b 1^{2} p 3}{a 1 b 1 b 2+b 1^{2} a 2+b 2^{2}}\right)
$$

For this controller we had tried the following different combinations of the poles.

The resulted graphs are seen in the Appendix-7.

At $2 \mathrm{e}^{-6}$, the following pole combination are consider

$q 1=0.48, q 2=0.46, \& q 3=0.47$

$q 1=0.21, q 2=0.24, \& q 3=0.25$ and

$q 1=0.38, q 2=0.36, \& q 3=0.37$

At this output $2 \mathrm{e}^{-6}$, and at poles $\mathrm{q} 1=0.38, \mathrm{q} 2=0.36, \& \mathrm{q} 3=0.37$, the result is better than the remaining combinations, so we consider these pole values for both the controllers. And the same formula is used as in equation 26. 


\subsection{Simulation results of the Continuous and Discrete model of PD controller:}

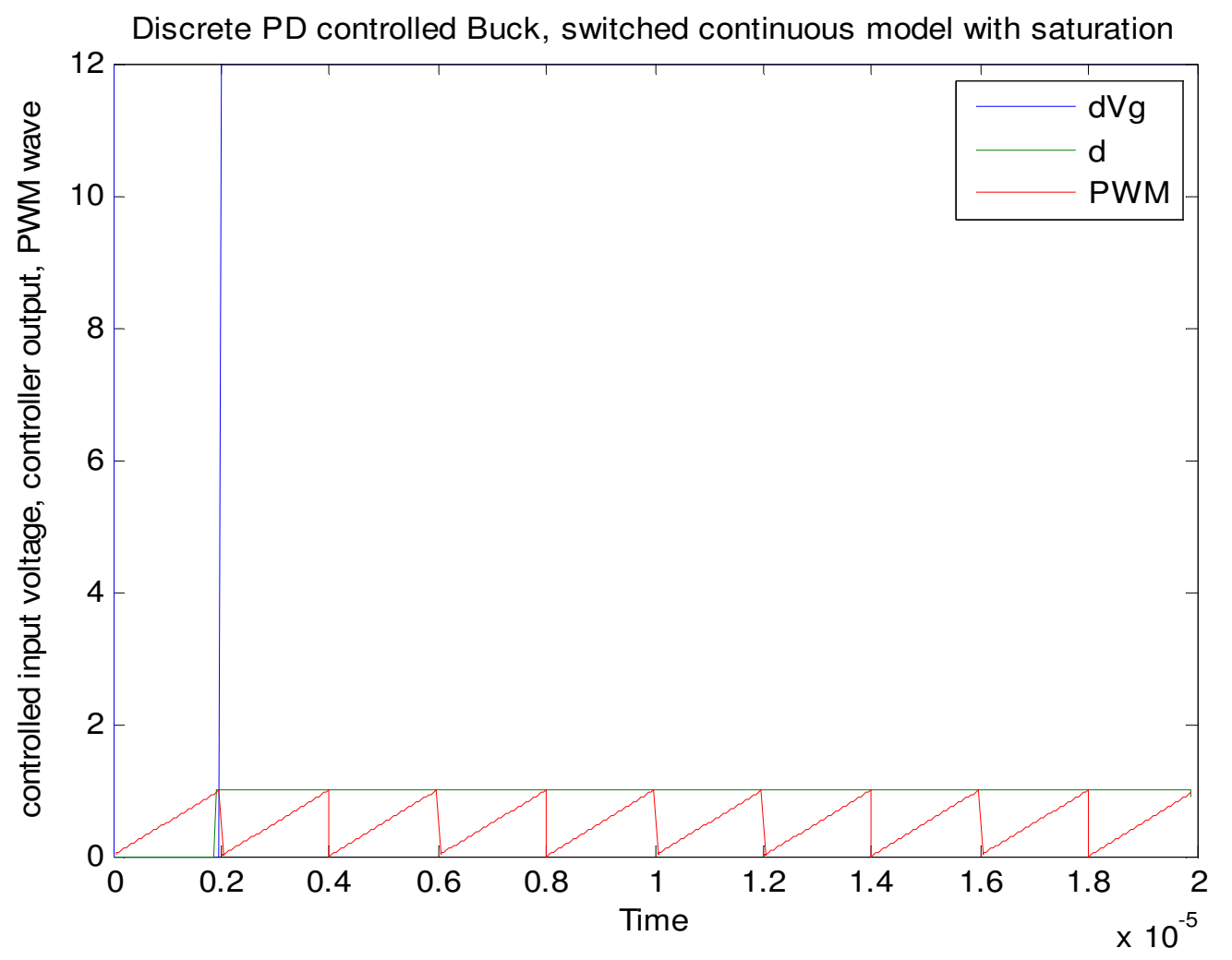

Fig 36: Discrete PD controlled buck, switched continuous model with saturation.

In the above figure we can observe that the $d$ signal is constant with the pwm signal, from this we can say the system is stable with reference to the pwm signal. And now we can consider the output graphs for the continuous and discrete models. 


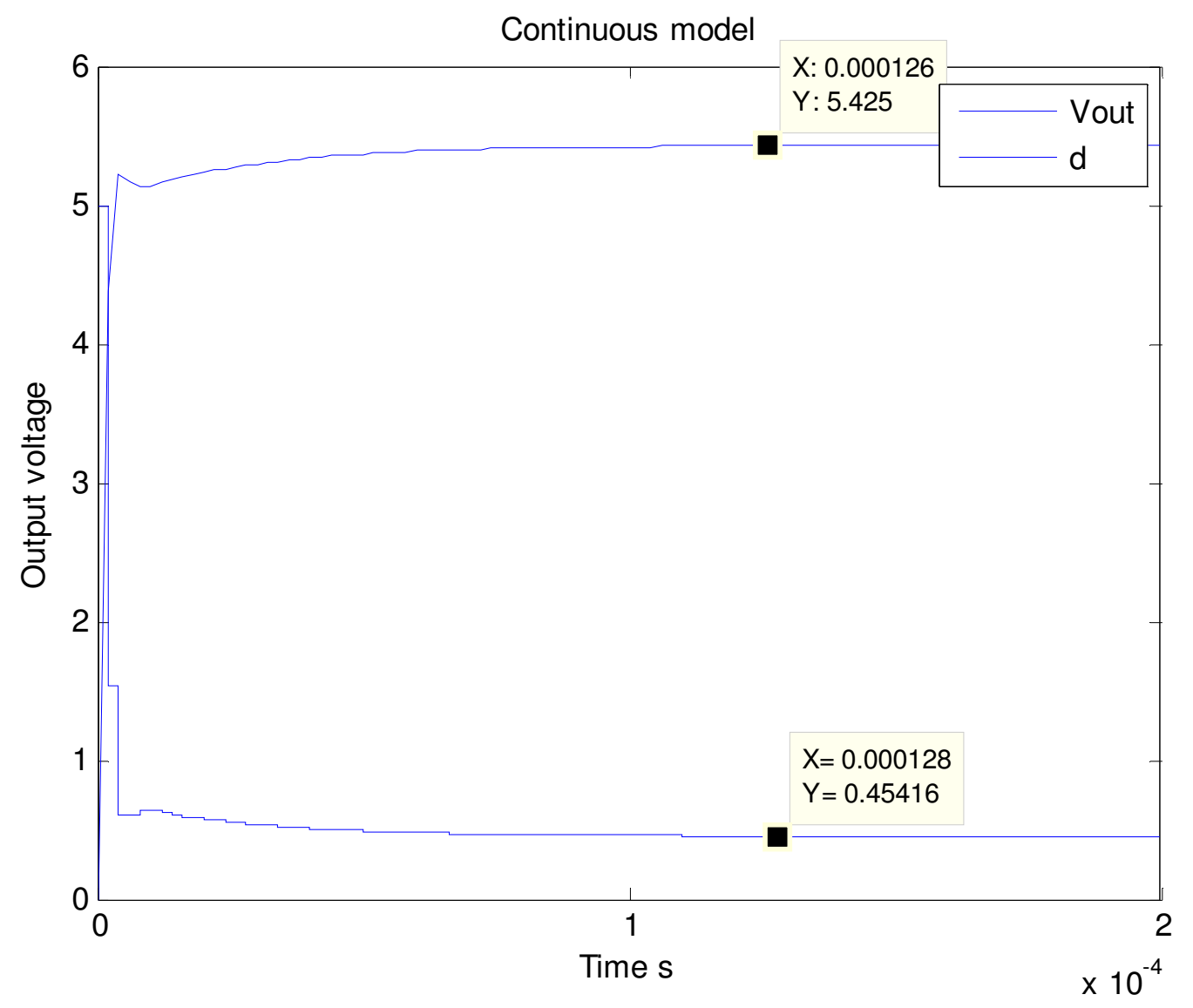

Fig: 37 continue model, system output and controller output.

In this simulation [continuous model] the reference is constant at $5.4 \mathrm{v}$, which is nearer to the expected reference voltage 5 . And the output voltage also is constant from same as the' $d^{\prime}$ signal, it shows that the continuous model with PD controller works with little late response.

The controller output can get stable quickly [in $1.3 \mu \mathrm{s}$ ] and remain stable. What's more, the controller make the output voltage come to stable status quickly [in $1.3 \mu \mathrm{s}$ ] and remain the wanted output voltage nearer to the reference voltage $5 \mathrm{~V}$. 


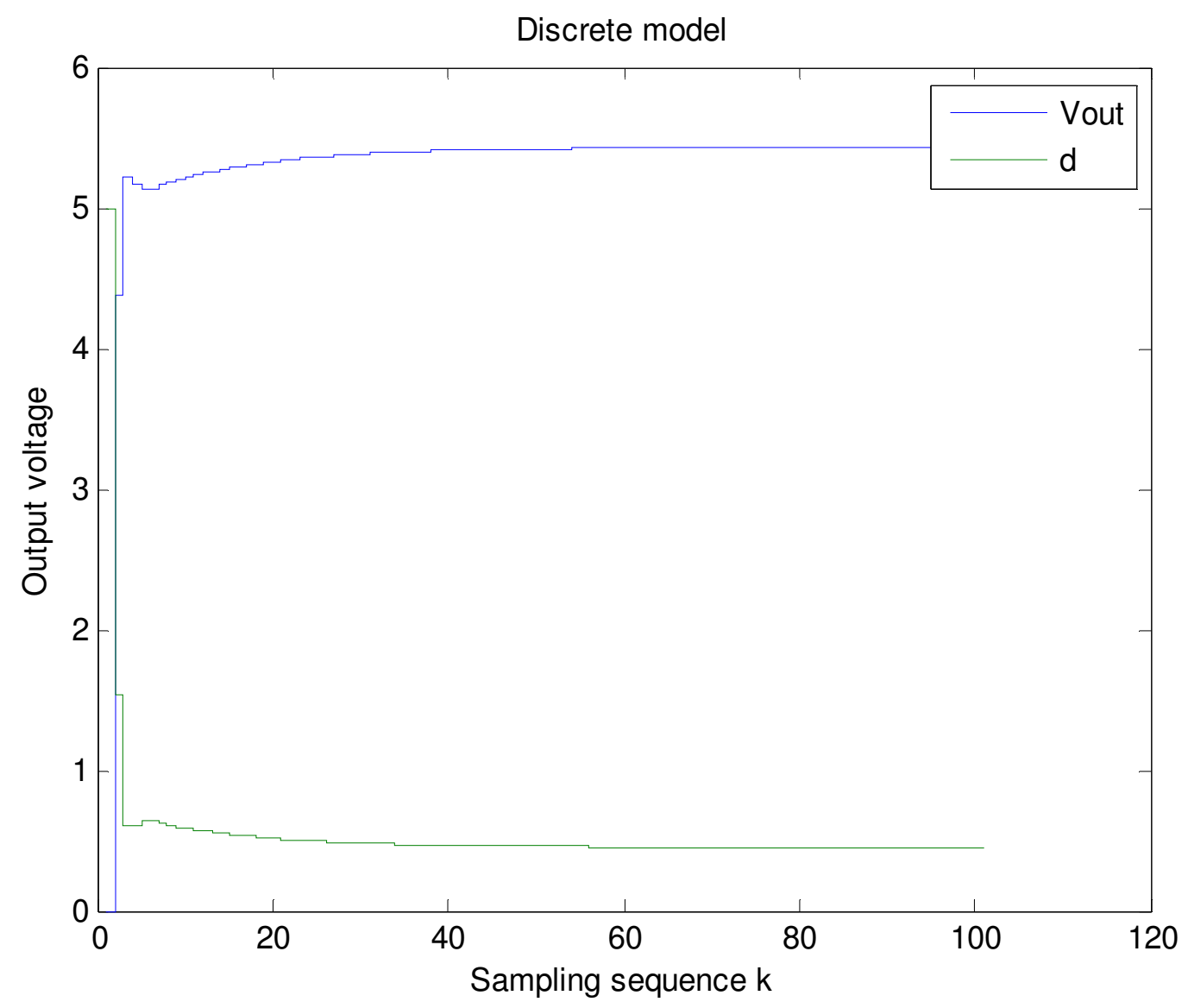

Fig 38: discrete model simulation for the PD controller.

It can be seen that the output voltage of discrete system can satisfy the required voltage. The controller output is stable from the 63 sampling interval. What's more, the controller make the output voltage come to stable quickly and remain the wanted output voltage [reference voltage $5 \mathrm{~V}]$.

The simulation is according to the polynomial controller theory, so it is an illustration of the theory by numerical calculations. When designing the controller, it should not hit the saturation limits too much for a reasonable disturbance. And the simulation result satisfies it. 


\subsection{Simulation result without disturbance and with noise:}

In the real case, the circuit consists of some noise, at the switch according to high frequency switching, measurement noise and so on. Here, adding some band-limited white noise to simulate it.

The noise magnitude is between 5.3 and 5.5 , for the simulation results see the below figure.

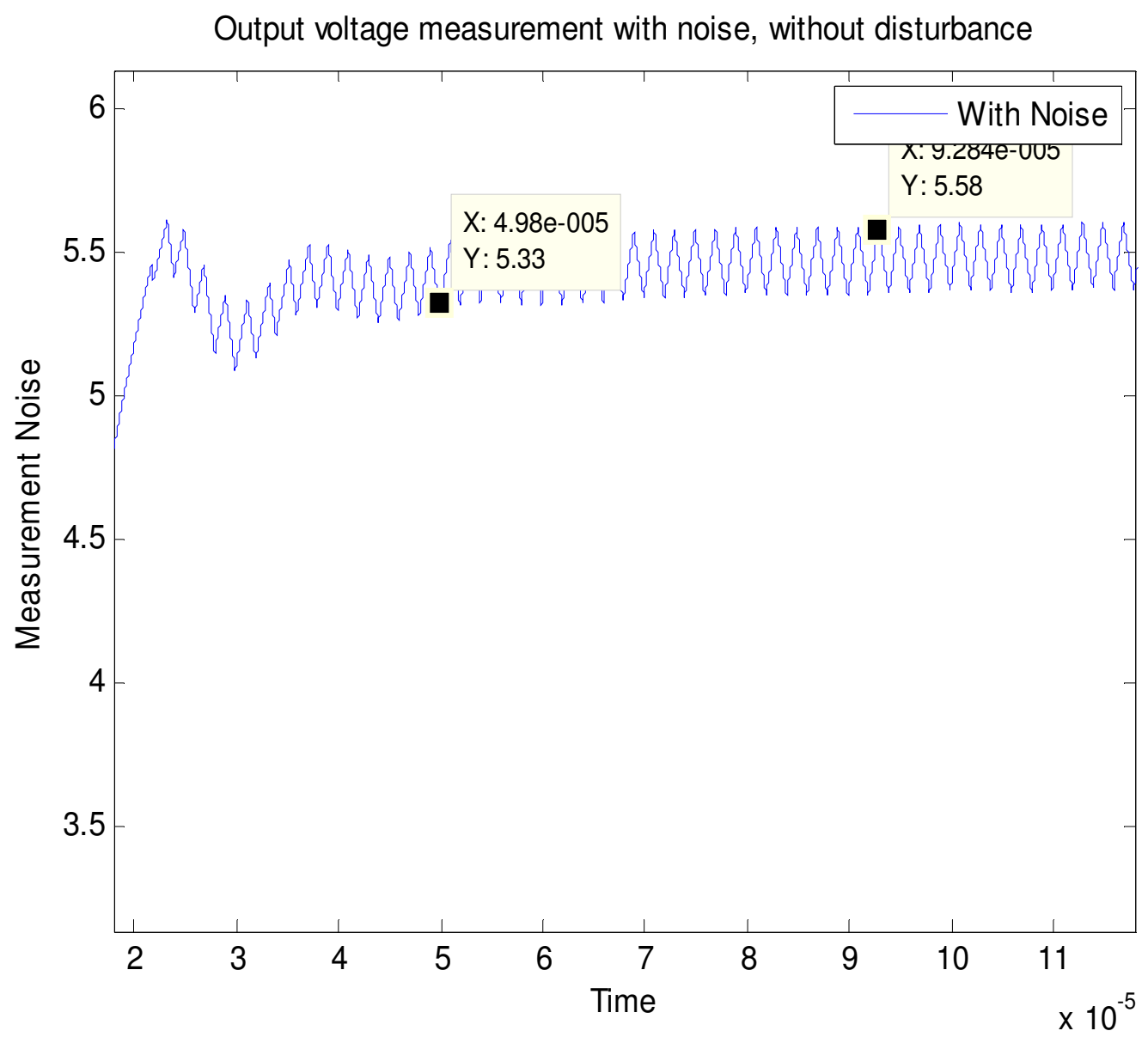

Fig 39: output voltage measurement with noise, and without disturbance.

\subsection{Simulation results without noise and with disturbance:}

Here current source is used as the load, when the output current suddenly changes, then their will be some disturbance in the output voltage too. 
To observe the disturbance and check whether the polynomial controller has enough ability to turn the output voltage back to reference voltage. Setting the current change range as 5A-15A. we can observe the simulation results below.

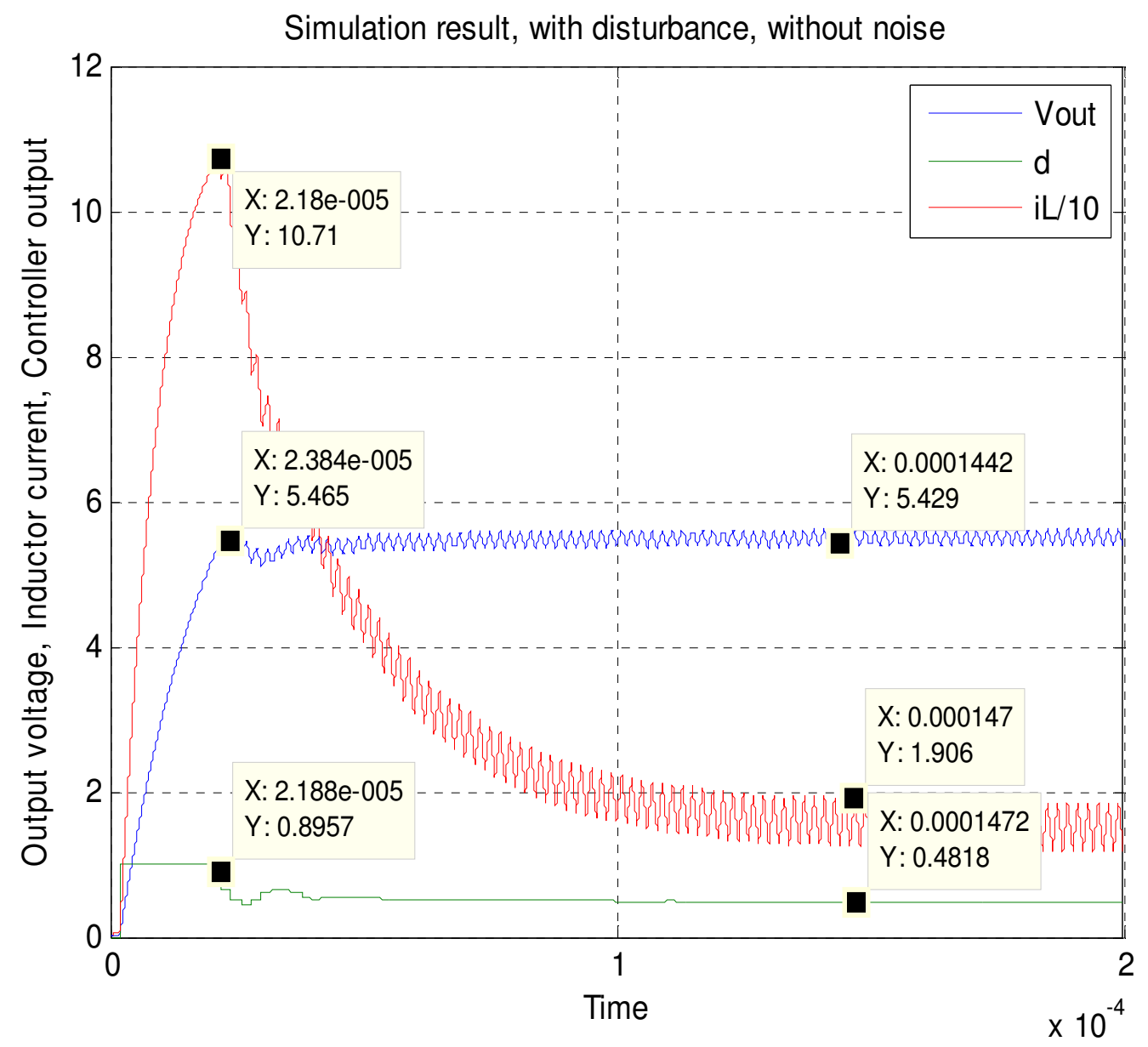

Fig 40: simulation result, with disturbance and without noise.

The stable output voltage under control is about $5.44 \mathrm{~V}$ which is quite close to the reference voltage $5 \mathrm{~V}$, and it means the controller is good.

At the 60th sample interval [180 $\mu$ s] changing the load current from $5 A$ to $15 A$, the output voltage does not fall or rise the voltage, maintains same voltage 5.4 which very good because when the load changes suddenly the controller maintains the output voltage in a stable voltage. 


\section{Chapter 7. CONCLUSION:}

In this paper, three different controllers are designed and proposed for Ericsson's BMR450 [DCDC Buck Converter]. A Simulink model of the buck converter has been designed to generate the output needed for buck system, to get the required output from the system, a reference voltage was used. Here reference voltage is the key parameter for checking the performance of these controllers, and the controller blocks are implemented by using Matlab and substantiated through simulation results.

The designing method is same for all the three controllers i.e., Polynomial Pole Placement, PID and PD. While the polynomial controller involves straight forward design using the plant $B(z) / A(Z)$ and parameters $c 1, c 2, d 0, d 1$ and $d 2$. But in the digital PID controller, we have considered the design using three parameters - proportional, integral and derivative. And the parameters of $C$ and $D$ i.e., $r 0, r 1, r 2, s 1$ and $s 2$ are expressed in terms of PID, five parameters as $c 1, c 2, d 0, d 1$ and $d 2$ in PID we used same parameters as r0, r1, r2, s1 and s2. And for the PD controller we have considered the design using two parameters - proportional and derivative, here we have $\mathrm{c} 1, \mathrm{~d} 0$ and $\mathrm{d} 1$.

As per the results point of view, the polynomial performed well than PID and PD controllers. The simulations show that the polynomial controller reaches the reference voltage very well, were the PID and PD result does not differ very much while meeting with the required reference voltage. Thus we conclude that the Polynomial controller design and results were better than the PID and PD Controllers.

If we compare both the second order [4] and third order controller methods, The second order controllers are easy in design and gives better responses than third order polynomial PID and PD controllers.

\subsection{Future work:}

The performance could be tested by using few other controllers like RLS [Recursive Least Square] algorithm, LMS [Least Mean Square] algorithm. And the PID, PD Controller could be redesigned by using root locus method. The reduced order model could be used for testing the behavior of the buck converter. 


\section{Chapter 8. References:}

[1] Robert W. Erickson and Dragan Maksimovic, Fundamentals of power electronics, 2nd ed., New York: Kluwer Academic Publishers, 2004, pp. 1-2.

[2] Anirban Dashgupta and Antip Ghosh, "Study and design of buck converter", in Electrical Engg, NIT, Rourkel. Available: http://www.scribd.com/doc/19037931/Buck-Converter, verified on 25-June-2012.

[3] Robert W. Erickson, "DC-DC Power Converters", Article in Wiley Encyclopedia of Electrical and Electronics Engineering. Available:

[4] Yongseok Choi, Naehyuck Chang, Taewhan Kim, "DC-DC Converter-Aware Power Management for Low-Power Embedded Systems", in Computer- Aided Design of Integrated Circuits and Systems, IEEE Transactions, Vol. 26, pp. 1367 - 1381, Aug-2007.

[5] Shun Yang, "Modeling and control of a buck converter", Electrical Engineering with Emphasis on Telecommunication, from Blekinge Institute of Technology [BTH], ING/School of Engineering.

http://www.bth.se/fou/cuppsats.nsf/04c233cb0d90bad0c1256cec00325988/e6f4ba47896f169 9c12578b700388518! OpenDocument verified on 25-June-2012.

[6] K.Astrom and T.Hagglund; PID Controllers Theory, Design and Tuning, second edition 1995; The Instrumentation System and Automation Society, ISBN-10:1556175167.

[7] Bengt Schmidtbauer; Analog and digital control engineering; 1988; Student literature; Lund, Sweden; ISBN: 91-44-26601-4.

[8] Bengt Lennartson/ Bertil Thomas,"Analog and Digital Control Engineering" Education student housing literature, lund, Sweden ISBN: 91-44-26941-2.pp. 138-141.

[9] Loan D. Landau, Gianluca Zito; "Digital control systems" ISBN-10: 1846280559, 2006, pp. 106-115. 


\section{Appendix-1: Mathematical equation of buck converter when switch is} ON. (Page-16)

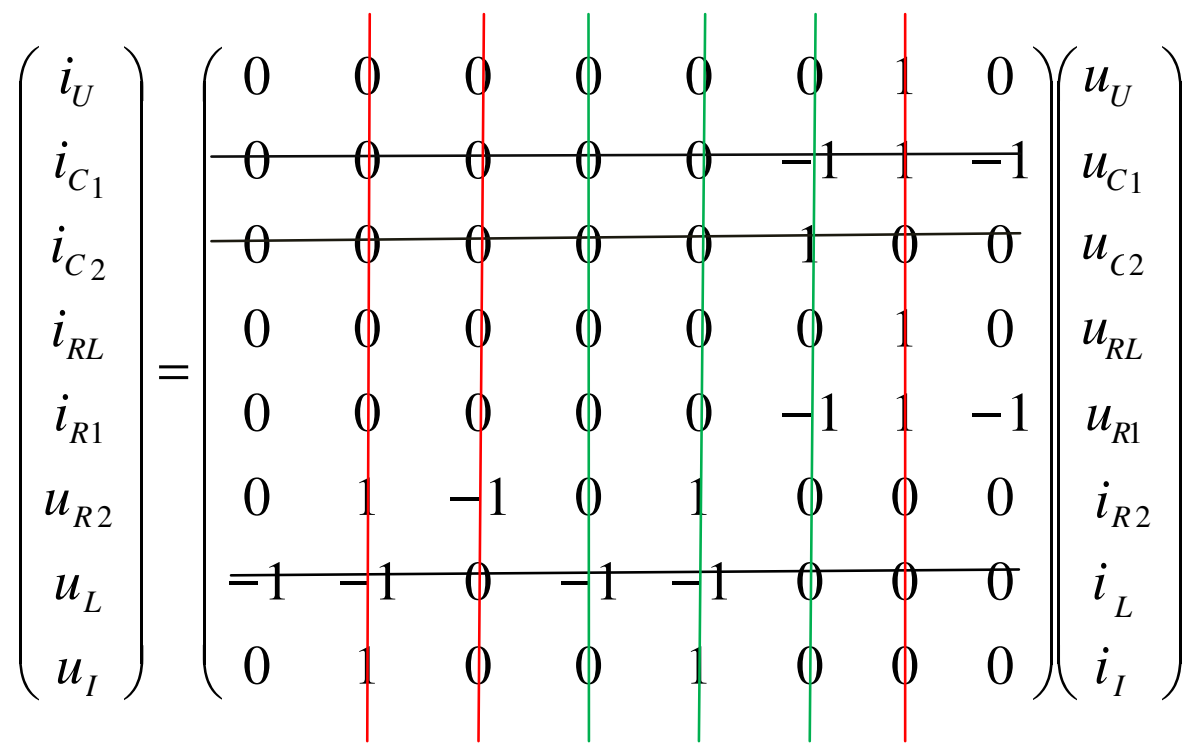

Matrix 4: Division of the matrix to transform in to $x$ dot $=A x+B u$.

So from the above matrix [8x8], transforming it to $x$ dot $=A x+B u$ equation, in Matrix 4 were the red and black lines meet those values are taken to represent the below matrix in [3x3] for $u_{C 1}, u_{C 2}, i_{L}$. And for the $u_{R L}, u_{R 1}, i_{R 2}$ matries are taken from the above Matrix 4 , were the green and black lines meet each other. The last matrix is $u_{U}$ and $i_{1}[2 \times 3]$ is consider from above matrix were $i_{C 1}[2 \times 1,2 \times 8], i_{C 2}[3 \times 1,3 \times 8], i_{L}[7 \times 1,7 \times 8]$.

$\left[\begin{array}{ccc}C_{1} & 0 & 0 \\ 0 & C_{2} & 0 \\ 0 & 0 & L\end{array}\right]\left[\begin{array}{c}\dot{u c_{1}} \\ \dot{u c_{2}} \\ \dot{i_{L}}\end{array}\right]=\left[\begin{array}{ccc}0 & 0 & 1 \\ 0 & 0 & 0 \\ -1 & 0 & 0\end{array}\right] \quad\left[\begin{array}{c}u_{C 1} \\ u_{C 2} \\ i_{L}\end{array}\right]+\left[\begin{array}{ccc}0 & 0 & -1 \\ 0 & 0 & 1 \\ -1 & -1 & 0\end{array}\right]\left[\begin{array}{c}u_{R L} \\ u_{R 1} \\ i_{R 2}\end{array}\right]+\left[\begin{array}{cc}0 & -1 \\ 0 & 0 \\ -1 & 0\end{array}\right]\left[\begin{array}{c}u_{U} \\ i_{I}\end{array}\right]$

$\left[\begin{array}{c}i_{R L} \\ i_{R 2} \\ u_{R 2}\end{array}\right]=\left[\begin{array}{ccc}0 & 0 & 1 \\ 0 & 0 & 1 \\ 1 & -1 & 0\end{array}\right]\left[\begin{array}{c}u_{C 1} \\ u_{C 2} \\ i_{L}\end{array}\right]+\left[\begin{array}{ccc}0 & 0 & 0 \\ 0 & 0 & -1 \\ 0 & 1 & 0\end{array}\right] \quad\left[\begin{array}{c}u_{R L} \\ u_{R 1} \\ i_{R 2}\end{array}\right]+\left[\begin{array}{cc}0 & 0 \\ 0 & -1 \\ 0 & 0\end{array}\right]\left[\begin{array}{c}u_{U} \\ i_{I}\end{array}\right]$

$\left[\begin{array}{c}i_{R L} \\ i_{R 1} \\ u_{R 2}\end{array}\right]=\left[\begin{array}{ccc}1 / R_{L} & 0 & 0 \\ 0 & 1 / R_{1} & 0 \\ 0 & 0 & R_{2}\end{array}\right]\left[\begin{array}{c}u_{R L} \\ u_{R 1} \\ i_{R 2}\end{array}\right]$

By substituting the equation $\mathbf{3}$ in $\mathbf{2}$, we obtain 


$$
\left[\begin{array}{ccc}
1 / R_{L} & 0 & 0 \\
0 & 1 / R_{1} & 0 \\
0 & 0 & R_{2}
\end{array}\right]\left[\begin{array}{c}
u_{R L} \\
u_{R 1} \\
i_{R 2}
\end{array}\right]=\left[\left[\begin{array}{ccc}
0 & 0 & 1 \\
0 & 0 & 1 \\
1 & -1 & 0
\end{array}\right]\left[\begin{array}{c}
u_{C 1} \\
u_{C 2} \\
i_{L}
\end{array}\right]+\left[\begin{array}{ccc}
0 & 0 & 0 \\
0 & 0 & -1 \\
0 & 1 & 0
\end{array}\right]\left[\begin{array}{c}
u_{R L} \\
u_{R 1} \\
i_{R 2}
\end{array}\right]+\left[\begin{array}{cc}
0 & 0 \\
0 & -1 \\
0 & 0
\end{array}\right]\left[\begin{array}{c}
u_{U} \\
i_{I}
\end{array}\right]\right)_{(4)}
$$

Solving the above equation in the following steps, as show below

$$
\left[\begin{array}{ccc}
0 & 0 & 0 \\
0 & 0 & 1 \\
0 & -1 & 0
\end{array}\right]\left[\begin{array}{ccc}
1 / R_{L} & 0 & 0 \\
0 & 1 / R_{1} & 0 \\
0 & 0 & R_{2}
\end{array}\right]\left[\begin{array}{l}
u_{R L} \\
u_{R 1} \\
i_{R 2}
\end{array}\right]=\left[\begin{array}{ccc}
0 & 0 & 1 \\
0 & 0 & 1 \\
1 & -1 & 0
\end{array}\right]\left[\begin{array}{c}
u_{C 1} \\
u_{C 2} \\
i_{L}
\end{array}\right]\left[\begin{array}{cc}
0 & 0 \\
0 & -1 \\
0 & 0
\end{array}\right]\left[\begin{array}{c}
u_{U} \\
i_{I}
\end{array}\right]
$$

$\left[\begin{array}{ccc}1 / R_{L} & 0 & 0 \\ 0 & 1 / R_{1} & 1 \\ 0 & -1 & R_{2}\end{array}\right]\left[\begin{array}{c}u_{R L} \\ u_{R 1} \\ i_{R 2}\end{array}\right]=\left[\begin{array}{ccc}0 & 0 & 1 \\ 0 & 0 & 1 \\ 1 & -1 & 0\end{array}\right]\left[\begin{array}{c}u_{C 1} \\ u_{C 2} \\ i_{L}\end{array}\right]+\left[\begin{array}{cc}0 & 0 \\ 0 & -1 \\ 0 & 0\end{array}\right]\left[\begin{array}{c}u_{U} \\ i_{I}\end{array}\right]$

$$
\left[\begin{array}{c}
u_{R L} \\
u_{R 1} \\
i_{R 2}
\end{array}\right]=\left[\begin{array}{ccc}
R_{L} & 0 & 0 \\
0 & R_{1} R_{2} /\left(R_{1}+R_{2}\right) & -R_{1} /\left(R_{1}+R_{2}\right) \\
0 & R_{1} /\left(R_{1}+R_{2}\right) & 1 /\left(R_{1}+R_{2}\right)
\end{array}\right]\left[\left[\begin{array}{ccc}
0 & 0 & 1 \\
0 & 0 & 1 \\
1 & -1 & 0
\end{array}\right]\left[\begin{array}{c}
u_{C 1} \\
u_{C 2} \\
i_{L}
\end{array}\right]+\left[\begin{array}{cc}
0 & 0 \\
0 & -1 \\
0 & 0
\end{array}\right]\left[\begin{array}{c}
u_{U} \\
i_{I}
\end{array}\right]\right)
$$

$$
\begin{aligned}
& {\left[\begin{array}{c}
u_{R L} \\
u_{R 1} \\
i_{R 2}
\end{array}\right]=\left[\begin{array}{ccc}
0 & 0 & R_{L} \\
-R_{1} /\left(R_{1}+R_{2}\right) & R_{1} /\left(R_{1}+R_{2}\right) & R_{1} R_{2} /\left(R_{1}+R_{2}\right) \\
1 /\left(R_{1}+R_{2}\right) & -1 /\left(R_{1}+R_{2}\right) & R_{1} /\left(R_{1}+R_{2}\right)
\end{array}\right]\left[\begin{array}{c}
u_{C 1} \\
u_{C 2} \\
i_{L}
\end{array}\right]+\left[\begin{array}{cc}
0 & 0 \\
0 & -R 1 R 2 /(R 1+R 2) \\
0 & -R 1 /(R 1+R 2)
\end{array}\right]\left[\begin{array}{c}
u_{U} \\
i_{I}
\end{array}\right]{ }_{(\mathbf{5})}} \\
& {\left[\begin{array}{c}
i_{C 1} \\
i_{C 2} \\
u_{L}
\end{array}\right]=\left[\begin{array}{ccc}
0 & 0 & 1 \\
0 & 0 & 0 \\
-1 & 0 & 0
\end{array}\right] \quad\left[\begin{array}{c}
u_{C 1} \\
u_{C 2} \\
i_{L}
\end{array}\right]+\left[\begin{array}{ccc}
0 & 0 & -1 \\
0 & 0 & 1 \\
-1 & -1 & 0
\end{array}\right]\left[\begin{array}{c}
u_{R L} \\
u_{R 1} \\
i_{R 2}
\end{array}\right]+\left[\begin{array}{cc}
0 & -1 \\
0 & 0 \\
-1 & 0
\end{array}\right]\left[\begin{array}{c}
u_{U} \\
i_{I}
\end{array}\right]}
\end{aligned}
$$

Substituting equation 5 in equation $\mathbf{6}$, the resulted equation is as show below. 


$$
\begin{aligned}
& {\left[\begin{array}{c}
i_{C 1} \\
i_{C 2} \\
u_{L}
\end{array}\right]=\left[\begin{array}{ccc}
0 & 0 & 1 \\
0 & 0 & 0 \\
-1 & 0 & 0
\end{array}\right]} \\
& {\left[\begin{array}{ccc}
{\left[\begin{array}{c}
u_{C 1} \\
u_{C 2} \\
i_{L}
\end{array}\right]+\left[\begin{array}{ccc}
0 & 0 & -1 \\
0 & 0 & 1 \\
-1 & -1 & 0
\end{array}\right]} \\
-R_{1} /\left(R_{1}+R_{2}\right) & R_{1} /\left(R_{1}+R_{2}\right) & R_{1} R_{2} /\left(R_{1}+R_{2}\right) \\
1 /\left(R_{1}+R_{2}\right) & -1 /\left(R_{1}+R_{2}\right) & R_{1} /\left(R_{1}+R_{2}\right)
\end{array}\right]\left[\begin{array}{c}
u_{C 1} \\
u_{C 2} \\
i_{L}
\end{array}\right]+\left[\begin{array}{cc}
0 & 0 \\
0 & -R 1 R 2 /(R 1+R 2) \\
0 & -R 1 /(R 1+R 2)
\end{array}\right]\left[\begin{array}{c}
u_{U} \\
i_{I}
\end{array}\right]} \\
& {\left[\begin{array}{cc}
0 & -1 \\
0 & 0 \\
-1 & 0
\end{array}\right]\left[\begin{array}{c}
u_{U} \\
i_{I}
\end{array}\right]}
\end{aligned}
$$

$$
\left[\begin{array}{ccc}
0 & 0 & R_{L} \\
-R_{1} /\left(R_{1}+R_{2}\right) & R_{1} /\left(R_{1}+R_{2}\right) & R_{1} R_{2} /\left(R_{1}+R_{2}\right) \\
1 /\left(R_{1}+R_{2}\right) & -1 /\left(R_{1}+R_{2}\right) & R_{1} /\left(R_{1}+R_{2}\right)
\end{array}\right] \text { And this matrix }\left[\begin{array}{cc}
0 & 0 \\
0 & -R 1 R 2 /(R 1+R 2) \\
0 & -R 1 /(R 1+R 2)
\end{array}\right] \text { we get }
$$
the below equation

$$
\begin{aligned}
{\left[\begin{array}{c}
i_{C 1} \\
i_{C 2} \\
u_{L}
\end{array}\right]=} & {\left[\begin{array}{ccc}
0 & 0 & 1 \\
0 & 0 & 0 \\
-1 & 0 & 0
\end{array}\right]\left[\begin{array}{c}
u_{C 1} \\
u_{C 2} \\
i_{L}
\end{array}\right]+\left[\begin{array}{ccc}
-1 /\left(R_{1}+R_{2}\right) & 1 /\left(R_{1}+R_{2}\right) & -R_{1} /\left(R_{1}+R_{2}\right) \\
1 /\left(R_{1}+R_{2}\right) & -1 /\left(R_{1}+R_{2}\right) & R_{1} /\left(R_{1}+R_{2}\right) \\
R_{1} /\left(R_{1}+R_{2}\right) & -R_{1} /\left(R_{1}+R_{2}\right) & -R_{L}-R 1 R_{1} /\left(R_{1}+R_{2}\right)
\end{array}\right]\left[\begin{array}{c}
u_{C 1} \\
u_{C 2} \\
i_{L}
\end{array}\right]+} \\
& {\left[\begin{array}{cc}
0 & R_{1} /\left(R_{1}+R_{2}\right) \\
0 & -R_{1} /\left(R_{1}+R_{2}\right) \\
0 & R_{1} R_{2} /\left(R_{1}+R_{2}\right)
\end{array}\right]\left[\begin{array}{c}
u_{U} \\
i_{I}
\end{array}\right]+\left[\begin{array}{cc}
0 & -1 \\
0 & 0 \\
-1 & 0
\end{array}\right]\left[\begin{array}{c}
u_{U} \\
i_{I}
\end{array}\right] }
\end{aligned}
$$

$$
\begin{aligned}
& {\left[\begin{array}{c}
i_{C 1} \\
i_{C 2} \\
u_{L}
\end{array}\right]=\left[\begin{array}{ccc}
-1 /\left(R_{1}+R_{2}\right) & 1 /\left(R_{1}+R_{2}\right) & 1-R_{1} /\left(R_{1}+R_{2}\right) \\
1 /\left(R_{1}+R_{2}\right) & -1 /\left(R_{1}+R_{2}\right) & R_{1} /\left(R_{1}+R_{2}\right) \\
-1+R_{1} /\left(R_{1}+R_{2}\right) & -R_{1} /\left(R_{1}+R_{2}\right) & -R_{L}-R 1 R_{1} /\left(R_{1}+R_{2}\right)
\end{array}\right]\left[\begin{array}{c}
u_{C 1} \\
u_{C 2} \\
i_{L}
\end{array}\right]+} \\
& {\left[\begin{array}{cc}
0 & -1+R_{1} /\left(R_{1}+R_{2}\right) \\
0 & -R_{1} /\left(R_{1}+R_{2}\right) \\
-1 & R_{1} R_{2} /\left(R_{1}+R_{2}\right)
\end{array}\right]\left[\begin{array}{c}
u_{U} \\
i_{I}
\end{array}\right]}
\end{aligned}
$$


The above equation is the state space model of the signal.

The output voltage equation is $u_{0}=u_{R 1}+u_{C 1}$

Here considering the $U_{R 1}$ from 3 matrix row in equation $\mathbf{5}$ and $U_{C 1}$ matrix from equation $\mathbf{6}$

$$
\left[\begin{array}{c}
u_{R L} \\
u_{R 1} \\
i_{R_{2}}
\end{array}\right]=\left[\begin{array}{ccc}
0 & 0 & R_{L} \\
-R_{1} /\left(R_{1}+R_{2}\right) & R_{1} /\left(R_{1}+R_{2}\right) & R_{1} R_{2} /\left(R_{1}+R_{2}\right) \\
1 /\left(R_{1}+R_{2}\right) & -1 /\left(R_{1}+R_{2}\right) & R_{1} /\left(R_{1}+R_{2}\right)
\end{array}\right]\left[\begin{array}{c}
u_{C 1} \\
u_{C 2} \\
i_{L}
\end{array}\right]+\left[\begin{array}{cc}
0 & 0 \\
0 & -R 1 R 2 /(R 1+R 2) \\
0 & -R 1 /(R 1+R 2)
\end{array}\right]\left[\begin{array}{c}
u_{U} \\
i_{I}
\end{array}\right]
$$

$$
\begin{aligned}
& \mathrm{u}_{\mathrm{R} 1}=\left[\begin{array}{ccc}
{\left[-R_{1} /\left(R_{1}+R_{2}\right)\right.} & R_{1} /\left(R_{1}+R_{2}\right) & R_{1} R_{2} /\left(R_{1}+R_{2}\right)
\end{array}\right]\left[\begin{array}{c}
u_{C 1} \\
u_{C 2} \\
i_{L}
\end{array}\right]+\left[\begin{array}{ll}
0 & -R_{1} R_{2} /\left(R_{1}+R_{2}\right)
\end{array}\right] \\
& {\left[\begin{array}{c}
u U \\
i_{I}
\end{array}\right]+\mathrm{U}_{\mathrm{C} 1}} \\
& \mathrm{u}_{\mathrm{R} 1}=\left[1-R_{1} /\left(R_{1}+R_{2}\right) \quad R_{1} /\left(R_{1}+R_{2}\right) \quad R_{1} R_{2} /\left(R_{1}+R_{2}\right)\right]\left[\begin{array}{c}
u_{C 1} \\
u_{C 2} \\
i_{L}
\end{array}\right]+\left[\begin{array}{ll}
0 & -R_{1} R_{2} /\left(R_{1}+R_{2}\right)
\end{array}\right]\left[\begin{array}{c}
u_{U} \\
i_{I}
\end{array}\right]
\end{aligned}
$$

And changing $u$ into $v$ for the input voltage $[v=-u]$.

$$
\begin{aligned}
{\left[\begin{array}{c}
i_{C 1} \\
i_{C 2} \\
u_{L}
\end{array}\right]=} & {\left[\begin{array}{ccc}
-1 /\left(R_{1}+R_{2}\right) & 1 /\left(R_{1}+R_{2}\right) & 1-R_{1} /\left(R_{1}+R_{2}\right) \\
1 /\left(R_{1}+R_{2}\right) & -1 /\left(R_{1}+R_{2}\right) & R_{1} /\left(R_{1}+R_{2}\right) \\
-1+R_{1} /\left(R_{1}+R_{2}\right) & -R_{1} /\left(R_{1}+R_{2}\right) & -R_{L}-R 1 R_{1} /\left(R_{1}+R_{2}\right)
\end{array}\right]\left[\begin{array}{c}
u_{C 1} \\
u_{C 2} \\
i_{L}
\end{array}\right]+} \\
& {\left[\begin{array}{cc}
0 & -1+R_{1} /\left(R_{1}+R_{2}\right) \\
0 & -R_{1} /\left(R_{1}+R_{2}\right) \\
1 & R_{1} R_{2} /\left(R_{1}+R_{2}\right)
\end{array}\right]\left[\begin{array}{c}
v \\
i_{I}
\end{array}\right] }
\end{aligned}
$$


System equation:

$$
\begin{aligned}
{\left[\begin{array}{c}
u_{C 1} \\
u_{C 2} \\
i_{L}
\end{array}\right]=} & {\left[\begin{array}{ccc}
-1 /\left(R_{1}+R_{2}\right) C_{1} & 1 /\left(R_{1}+R_{2}\right) C_{1} & 1 / C_{1}-R_{1} /\left(R_{1}+R_{2}\right) C_{1} \\
1 /\left(R_{1}+R_{2}\right) C_{2} & -1 /\left(R_{1}+R_{2}\right) C_{2} & R_{1} /\left(R_{1}+R_{2}\right) C_{2} \\
-1 / L+R_{1} /\left(R_{1}+R_{2}\right) L & -R_{1} /\left(R_{1}+R_{2}\right) L & -R_{L}-R 1 R 1 /\left(R_{1}+R_{2}\right) L
\end{array}\right]\left[\begin{array}{c}
u_{C 1} \\
u_{C 2} \\
i_{L}
\end{array}\right]+} \\
& {\left[\begin{array}{cc}
0 & -1 / C_{1}+R_{1} /\left(R_{1}+R_{2}\right) C_{1} \\
0 & -R_{1} /\left(R_{1}+R_{2}\right) C_{2} \\
1 / L & R_{1} R_{2} /\left(R_{1}+R_{2}\right) L
\end{array}\right]\left[\begin{array}{c}
v \\
i_{I}
\end{array}\right] }
\end{aligned}
$$

Output equation:

$$
\left[1-R_{1} /\left(R_{1}+R_{2}\right) \quad R_{1} /\left(R_{1}+R_{2}\right) \quad R_{1} R_{2} /\left(R_{1}+R_{2}\right)\right]\left[\begin{array}{c}
u_{C 1} \\
u_{C 2} \\
i_{L}
\end{array}\right]+\left[\begin{array}{ll}
0 & -R_{1} R_{2} /\left(R_{1}+R_{2}\right)
\end{array}\right]\left[\begin{array}{c}
u_{U} \\
i_{I}
\end{array}\right]
$$




\section{Appendix -2: Solving the equation for obtaining the Polynomial pole placement. (Page-21)}

Recalling equation $\mathbf{2 2}$, and identifying the polynomials $A C+B D$ with $P$.

$$
\begin{aligned}
& C(z)=1+c_{1} z^{-1}+c_{2} z^{-2} \\
& P 1=c 1+a 1+b 1 d 0 \\
& P 2=c 2+a 2+a 1 c 1+b 1 d 1+b 2 d 0 \\
& P 3=a 1 c 2+a 2 c 1+a 3+b 1 d 2+b 2 d 1+b 3 d 0 \\
& P 4=a 2 c 2+a 3 c 1+b 2 d 2+b 3 d 1 \\
& P 5=a 3 c 2+b 3 d 2
\end{aligned}
$$

Solve the above equations by using matrix method.

$$
\begin{gathered}
\left(\begin{array}{ccccc}
1 & 0 & b 1 & 0 & 0 \\
a 1 & 1 & b 2 & b 1 & 0 \\
a 2 & a 1 & b 3 & b 2 & b 1 \\
a 3 & a 2 & 0 & b 3 & b 2 \\
0 & a 3 & 0 & 0 & b 3
\end{array}\right)\left(\begin{array}{c}
c 1 \\
c 2 \\
d 0 \\
d 1 \\
d 2
\end{array}\right)= \\
\left(\begin{array}{c}
-a 1+(-q 5-q 4-q 3-q 2-q 1) \\
-a 2+q 4 q 5+q 3 q 5+q 3 q 4+q 2 q 5+q 2 q 4+q 2 q 3+q 1 q 5+q 1 q 4+q 1 q 3+q 1 q 2 \\
-a 3+(-q 3 q 4 q 5-q 2 q 4 q 5-q 2 q 3 q 5-q 2 q 3 q 4-q 1 q 4 q 5- \\
q 1 q 3 q 5-q 1 q 3 q 4-q 1 q 2 q 5-q 1 q 2 q 5-q 1 q 2 q 4-q 1 q 2 q 3) \\
q 2 q 3 q 4 q 5+q 1 q 3 q 4 q 5+q 1 q 2 q 4 q 5+q 1 q 2 q 3 q 5+q 1 q 2 q 3 q 4 \\
-q 1 q 2 q 3 q 4 q 5
\end{array}\right)
\end{gathered}
$$

For simplicity let matrix $M=\left(\begin{array}{ccccc}1 & 0 & b 1 & 0 & 0 \\ a 1 & 1 & b 2 & b 1 & 0 \\ a 2 & a 1 & b 3 & b 2 & b 1 \\ a 3 & a 2 & 0 & b 3 & b 2 \\ 0 & a 3 & 0 & 0 & b 3\end{array}\right), F=\left(\begin{array}{c}c 1 \\ c 2 \\ d 0 \\ d 1 \\ d 2\end{array}\right)$ and 


$$
N=\left(\begin{array}{c}
-a 1+(-q 5-q 4-q 3-q 2-q 1) \\
-a 2+q 4 q 5+q 3 q 5+q 3 q 4+q 2 q 5+q 2 q 4+q 2 q 3+q 1 q 5+q 1 q 4+q 1 q 3+q 1 q 2 \\
-a 3+(-q 3 q 4 q 5-q 2 q 4 q 5-q 2 q 3 q 5-q 2 q 3 q 4-q 1 q 4 q 5- \\
q 1 q 3 q 5-q 1 q 3 q 4-q 1 q 2 q 5-q 1 q 2 q 5-q 1 q 2 q 4-q 1 q 2 q 3) \\
q 2 q 3 q 4 q 5+q 1 q 3 q 4 q 5+q 1 q 2 q 4 q 5+q 1 q 2 q 3 q 5+q 1 q 2 q 3 q 4 \\
-q 1 q 2 q 3 q 4 q 5
\end{array}\right)
$$




\section{Appendix -3: Resulted graphs for determining the pole values in polynomial controller. (Page-23)}

When the poles are $q 1=0.1, q 2=0.2, q 3=0.3, q 4=0.4, q 5=0.5$. And at Sampling interval $6 e^{-6}$ the below graph is obtained which is not stable.

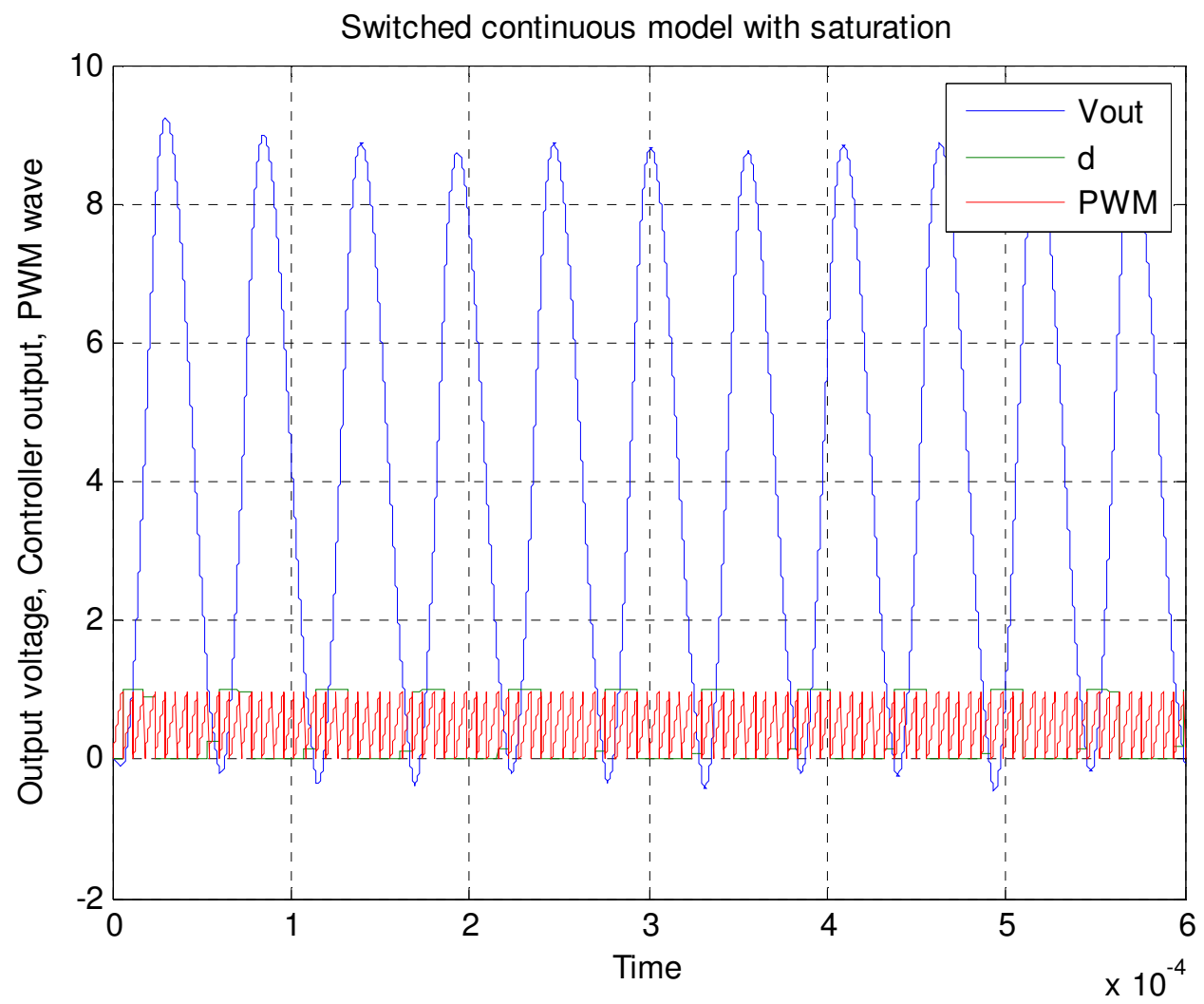

Figure 34: simulation of polynomial controller at poles $0.1,0.2,0.3,0.4$, and 0.5 at the sampling interval $6 \mathrm{e}^{-6}$.

And at the poles $q 1=0.3, q 2=0.4, q 3=0.5, q 4=0.6, q 5=0.7$ at the sampling interval $6 e^{-6}$ the resulted graph also not stable, as the graph with poles taken with 0.5 values. 


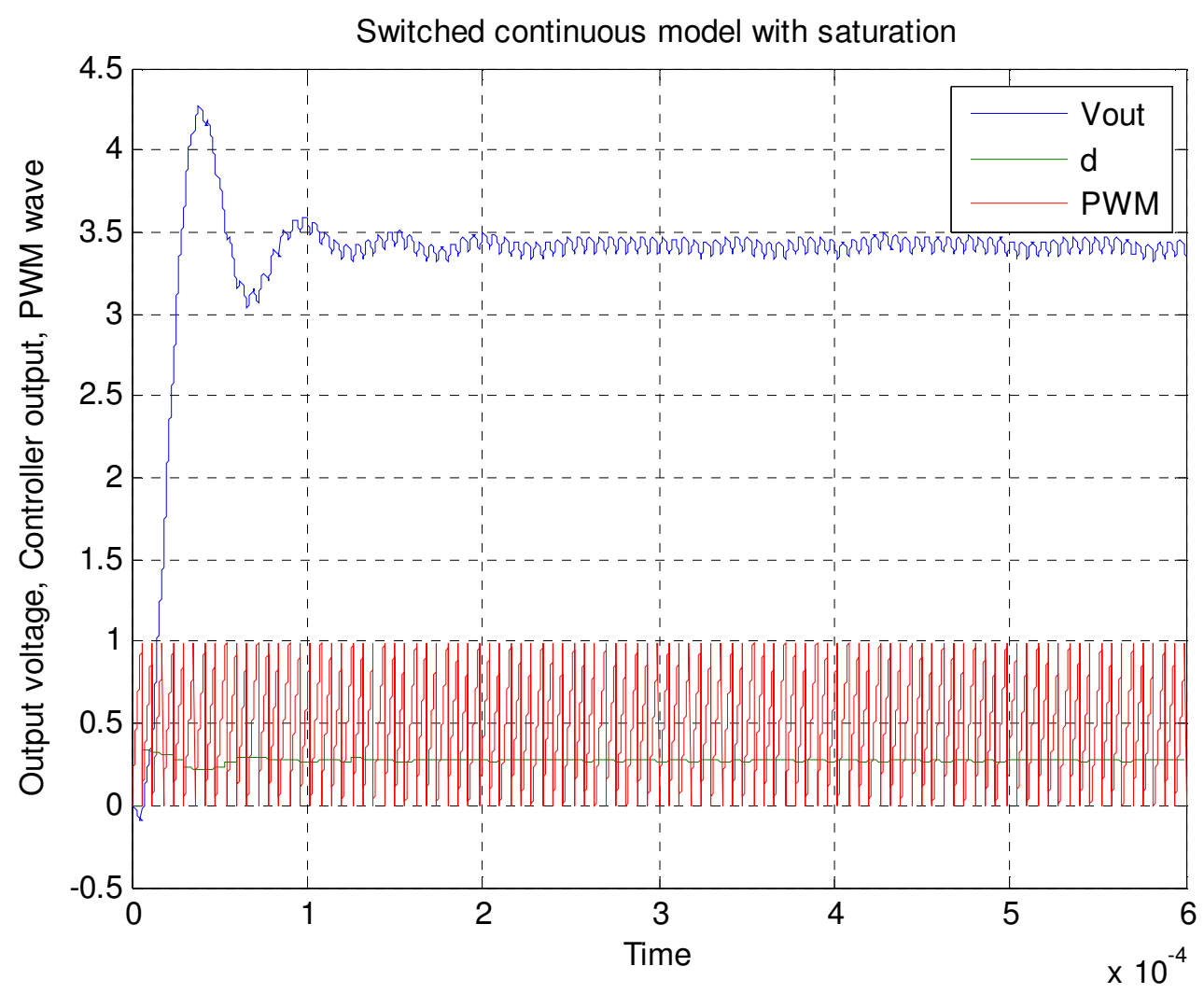

Figure 35: Simulation of polynomial controller at poles $0.3,0.4,0.5,0.6$, and 0.7 at the sampling interval $6 \mathrm{e}^{-6}$.

From the above resulted Figures 34 and 35, we had decided that, the consider poles values are not correct to stabilize the Polynomial Pole Placement controller. So we had taken the pole values to be $0.5,0.5,0.5,0.5$, and 0.5 at sampling interval $\left[6 \mathrm{e}^{-6}\right]$ is better. 


\section{Appendix -4: Solving the equation for obtaining the PID. (Page-41)}

Multiplying the above equation $\mathbf{5 8}$, gives

$$
\begin{aligned}
& = \\
& 1+s 1 z^{-1}+s 2 z^{-2}+a 1 z^{-1}+a 1 s 1 z^{-2}+a 1 s 2 z^{-3}+a 2 z^{-2}+a 2 s 1 z^{-3}+a 2 s 2 z^{-4}+ \\
& a 3 z^{-3}+a 3 s 1 z^{-4}+a 3 s 2 z^{-5}+b 1 r 0 z^{-1}+b 1 r 1 z^{-2}+b 1 r 2 z^{-3}+b 2 r 0 z^{-2}+ \\
& b 2 r 1 z^{-3}+b 2 r 2 z^{-4}+b 3 r 0 z^{-3}+b 3 r 1 z^{-4}+b 3 r 2 z^{-5}
\end{aligned}
$$

Taking as common in above equation.

$$
\begin{aligned}
& 1+(s 1+a 1+b 1 r 0) z^{-1}+(s 2+a 1 s 1+a 2+b 1 r 1+b 2 r 0) z^{-2}+(a 1 s 2+a 2 s 1+a 3+ \\
& b 1 r 2+b 2 r 1+b 3 r 0) z^{-3}+(a 2 s 2+a 3 s 1+b 2 r 2+b 3 r 1) z^{-4}+(a 3 s 2+b 3 r 2) z^{-5}
\end{aligned}
$$

Finding poles $\mathrm{P} 1, \mathrm{P} 2, \mathrm{P} 3, \mathrm{P} 4$, and $\mathrm{P} 5$ in terms of $\mathrm{q} 1, \mathrm{q} 2, \mathrm{q} 2, \mathrm{q} 4$ and $\mathrm{q} 5$.

$$
\begin{aligned}
& P\left(z^{-1}\right)=\left(1-q 1 z^{-1}\right)\left(1-q 2 z^{-1}\right)\left(1-q 3 z^{-1}\right)\left(1-q 4 z^{-1}\right)\left(1-q 5 z^{-1}\right)= \\
& \left.1+p 1 z^{-1}+p 2 z^{-2}+p 3 z^{-3}+p 4 z^{-4}+p 5 z^{-5}\right)
\end{aligned}
$$

Then poles would be,

$$
\begin{aligned}
& P 1=-q 5-q 4-q 3-q 2-q 1 \\
& P 2=q 4 q 5+q 3 q 5+q 3 q 4+q 2 q 5+q 2 q 4+q 2 q 3+q 1 q 5+q 1 q 4+q 1 q 3+q 1 q 2
\end{aligned}
$$

$$
\begin{aligned}
& P 3=-q 3 q 4 q 5-q 2 q 4 q 5-q 2 q 3 q 5-q 2 q 3 q 4-q 1 q 4 q 5- \\
& q 1 q 3 q 5-q 1 q 3 q 4-q 1 q 2 q 5-q 1 q 2 q 5-q 1 q 2 q 4-q 1 q 2 q 3 \\
& P 4=q 2 q 3 q 4 q 5+q 1 q 3 q 4 q 5+q 1 q 2 q 4 q 5+q 1 q 2 q 3 q 5+q 1 q 2 q 3 q 4 \\
& P 5=-q 1 q 2 q 3 q 4 q 5
\end{aligned}
$$

Recalling equation $\mathbf{2 7}$, and identifying the polynomial $A C+B D$ with $P$. 


$$
\begin{aligned}
& P 1=s 1+a 1+b 1 r 0 \\
& P 2=s 2+a 2+a 1 s 1+b 1 r 1+b 2 r 0 \\
& P 3=a 1 s 2+a 2 s 1+a 3+b 1 r 2+b 2 r 1+b 3 r 0 \\
& P 4=a 2 s 2+a 3 s 1+b 2 r 2+b 3 r 1 \\
& P 5=a 3 s 2+b 3 r 2
\end{aligned}
$$

Solve the above equations by using matrix method.

$$
\begin{gathered}
\left(\begin{array}{ccccc}
1 & 0 & b 1 & 0 & 0 \\
a 1 & 1 & b 2 & b 1 & 0 \\
a 2 & a 1 & b 3 & b 2 & b 1 \\
a 3 & a 2 & 0 & b 3 & b 2 \\
0 & a 3 & 0 & 0 & b 3
\end{array}\right)\left(\begin{array}{c}
s 1 \\
s 2 \\
r 0 \\
r 1 \\
r 2
\end{array}\right)= \\
\left(\begin{array}{c}
-a 1+(-q 5-q 4-q 3-q 2-q 1) \\
-a 2+q 4 q 5+q 3 q 5+q 3 q 4+q 2 q 5+q 2 q 4+q 2 q 3+q 1 q 5+q 1 q 4+q 1 q 3+q 1 q 2 \\
-a 3+(-q 3 q 4 q 5-q 2 q 4 q 5-q 2 q 3 q 5-q 2 q 3 q 4-q 1 q 4 q 5- \\
q 1 q 3 q 5-q 1 q 3 q 4-q 1 q 2 q 5-q 1 q 2 q 5-q 1 q 2 q 4-q 1 q 2 q 3) \\
q 2 q 3 q 4 q 5+q 1 q 3 q 4 q 5+q 1 q 2 q 4 q 5+q 1 q 2 q 3 q 5+q 1 q 2 q 3 q 4 \\
-q 1 q 2 q 3 q 4 q 5
\end{array}\right)
\end{gathered}
$$

(65)

The Matlab notation I used is $\mathrm{F}^{*} \mathrm{M}=\mathrm{J}=\mathrm{F}=\mathrm{inv}(\mathrm{M}) * \mathrm{~J}$, from this we can get the poles. 


\section{Appendix 5: Resulted graphs for determining the poles for the PID Controller. (Page-40)}

When the poles are $q 1=0.1, q 2=0.2, q 3=0.3, q 4=0.4, q 5=0.5$. And at sampling interval $1 e^{-6}$ the below graph is obtained which is not stable.

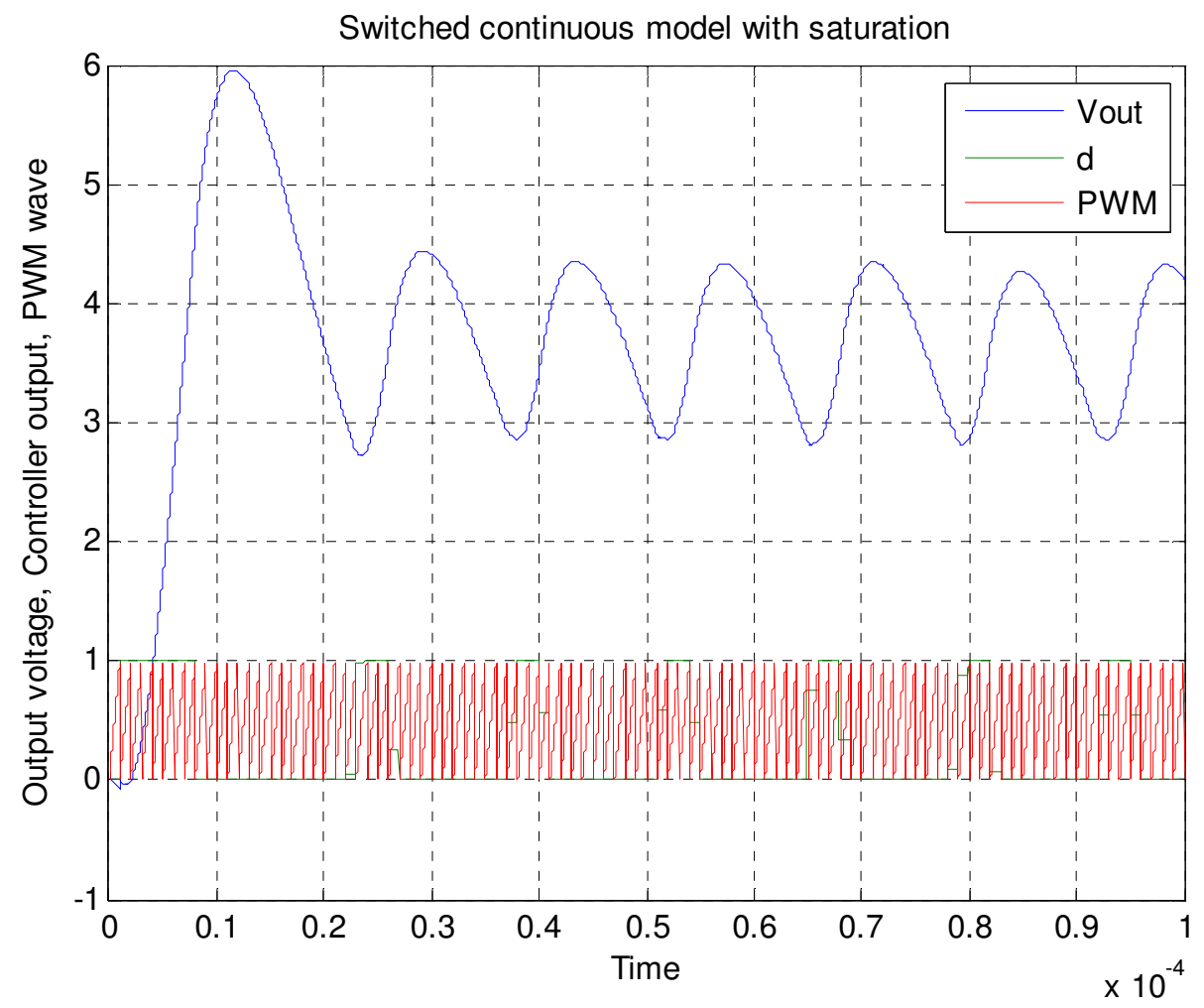

Figure 36: simulation of PID controller at poles $0.1,0.2,0.3,0.4$, and 0.5 at the sampling interval $1 \mathrm{e}^{-6}$.

And at the poles $\mathrm{q} 1=0.3, \mathrm{q} 2=0.4, \mathrm{q} 3=0.5, \mathrm{q} 4=0.6, \mathrm{q} 5=0.7$ at the sampling interval $1 \mathrm{e}^{-6}$ the resulted graph also not stable, as the graph with poles taken with 0.5 values. 


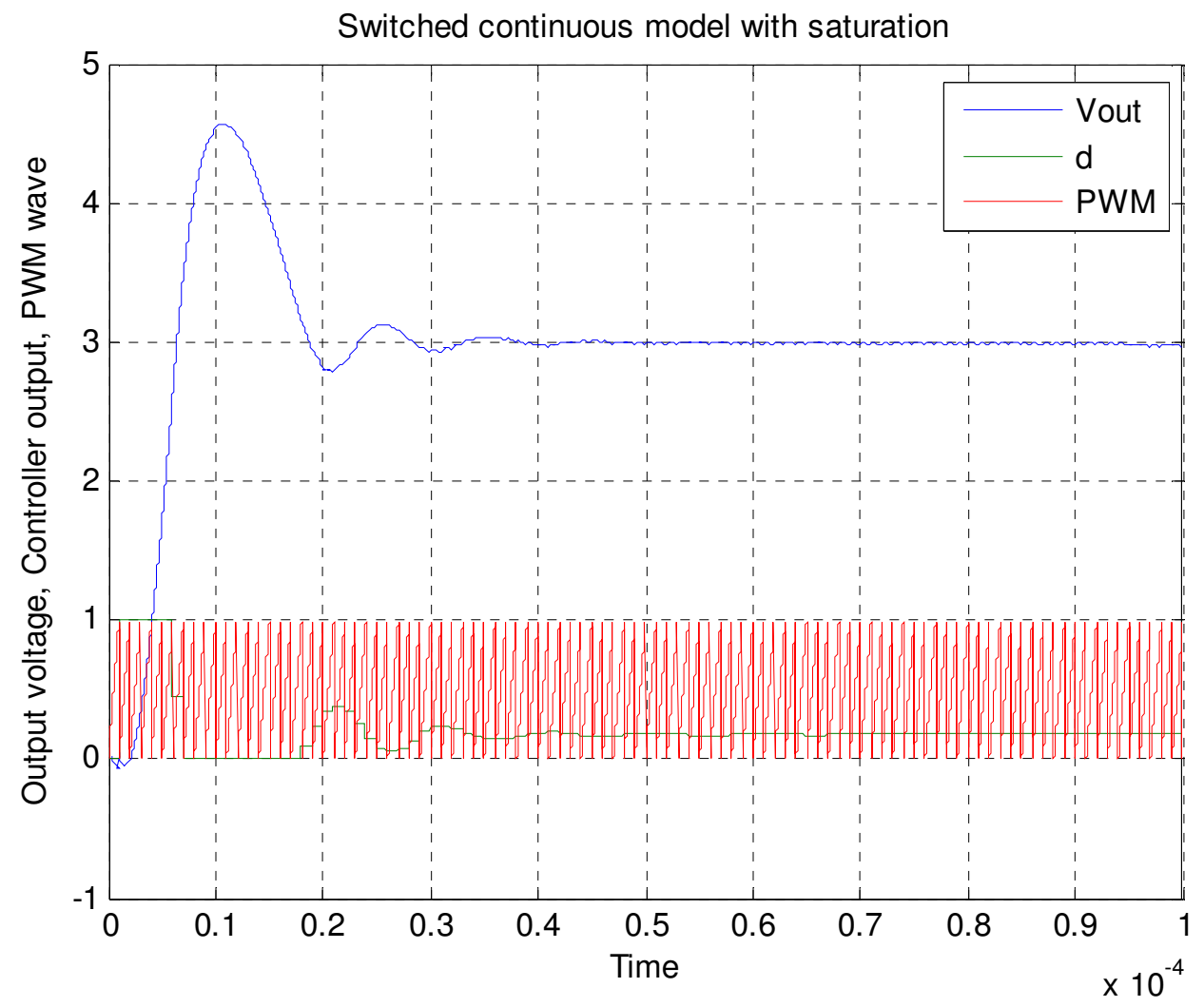

Figure 37: simulation of PID controller at poles $0.3,0.4,0.5,0.6$, and 0.7 at the sampling interval $1 \mathrm{e}^{-6}$.

From the above resulted Figures 36 and 37, we had decided that, the consider poles values for the PID controller are not correct to stabilize. So we had taken the pole values to be $0.5,0.5$, $0.5,0.5$, and 0.5 at sampling interval $\left[1 e^{-6}\right]$ is better. 
Appendix -6: Solving the equation for obtaining the PD (page-54)

$$
\begin{aligned}
& \mathrm{p} 2=\left(a 1 c 1+a 2+b 1\left(\frac{p 3-a 2 c 1}{b 2}\right)+b 2\left(\frac{p 1-c 1-a 1}{b 1}\right)\right) \\
& \mathrm{p} 2=\left(\frac{a 1 c 1 b 2 b 1+a 2 b 2 b 1+b 1^{2} p 3-b 1^{2} a 2 c 1+b 2^{2} p 1-c 1 b 2^{2}-a 1 b 2^{2}}{b 1 b 2}\right) \\
& p 2 b 1 b 2=c 1\left(a 1 b 2 b 1-b 1^{2} a 2-b 2^{2}\right)+a 2 b 2 b 1+b 1^{2} p 3+b 2^{2} p 1-a 1 b 2^{2} \\
& c 1=\left(\frac{b 1 b 2 p 2-a 2 b 2 b 1-b 1^{2} p 3+b 2^{2}(a 1-p 1)}{a 1 b 1 b 2-b 1^{2} a 2+b 2^{2}}\right) \\
& c 1=\left(\frac{b 1 b 2(p 2-a 2)+b 2^{2}(a 1-p 1)-b 1^{2} p 3}{a 1 b 1 b 2+b 1^{2} a 2+b 2^{2}}\right)
\end{aligned}
$$




\section{Appendix -7: Resulted graphs for determining the pole values in PD controller. (page54)}

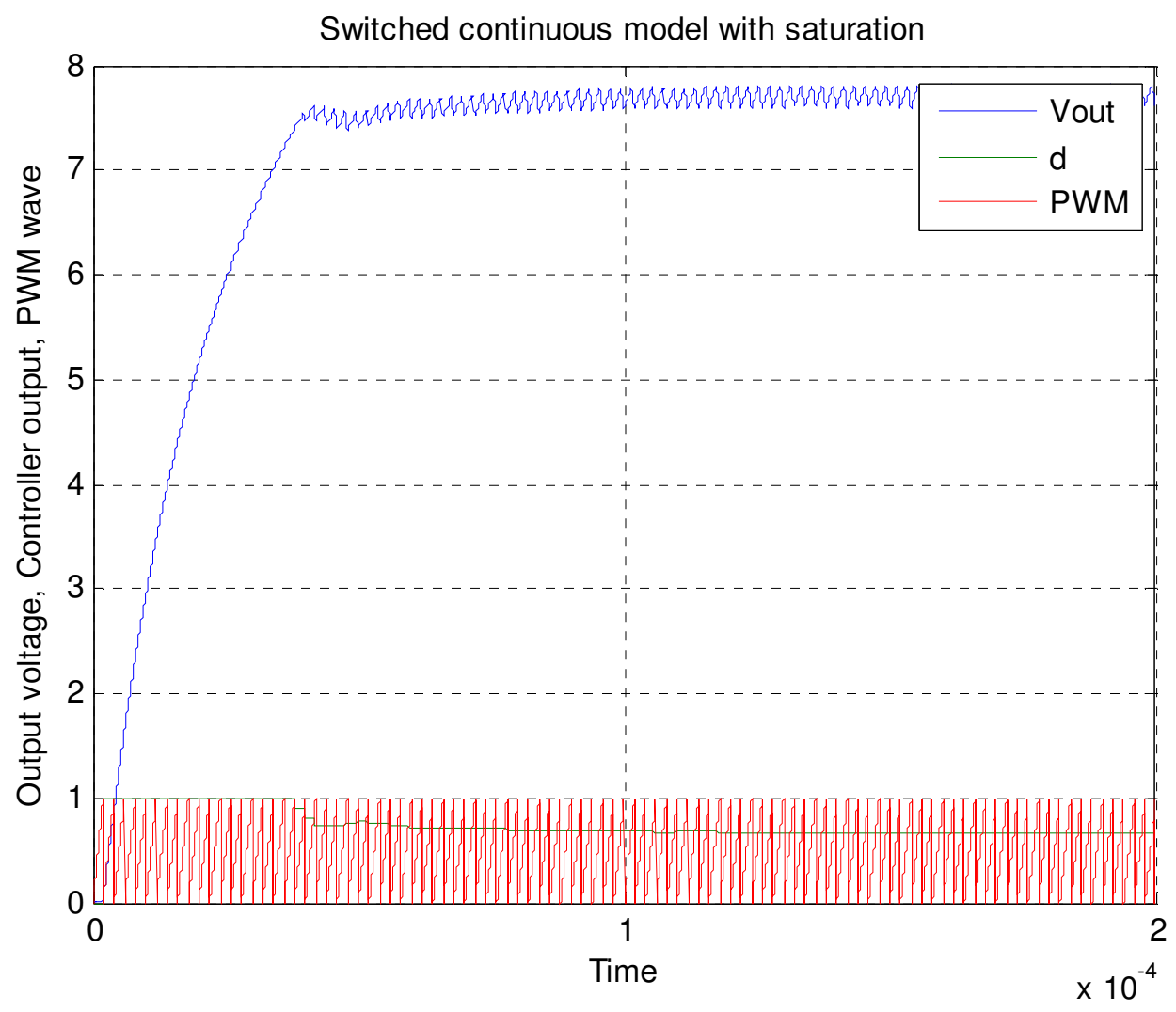

In the above graph, $v_{\text {out }}$ is constant 7.7 with oscillations, it doesn't fulfill our required expectations with the poles $0.48,0.46 \& 0.47$ at sampling interval $2^{\mathrm{e}-6}$, and if we consider the simulation results of the discrete model with saturation here $v_{\text {out }}$ is increasing up to $100 \mathrm{k}$ while the $d$ signal is constant from initial, so from observing these graphs [switched and discrete] we can observer that the reference value is not constant and is not at the required voltage 5 . 


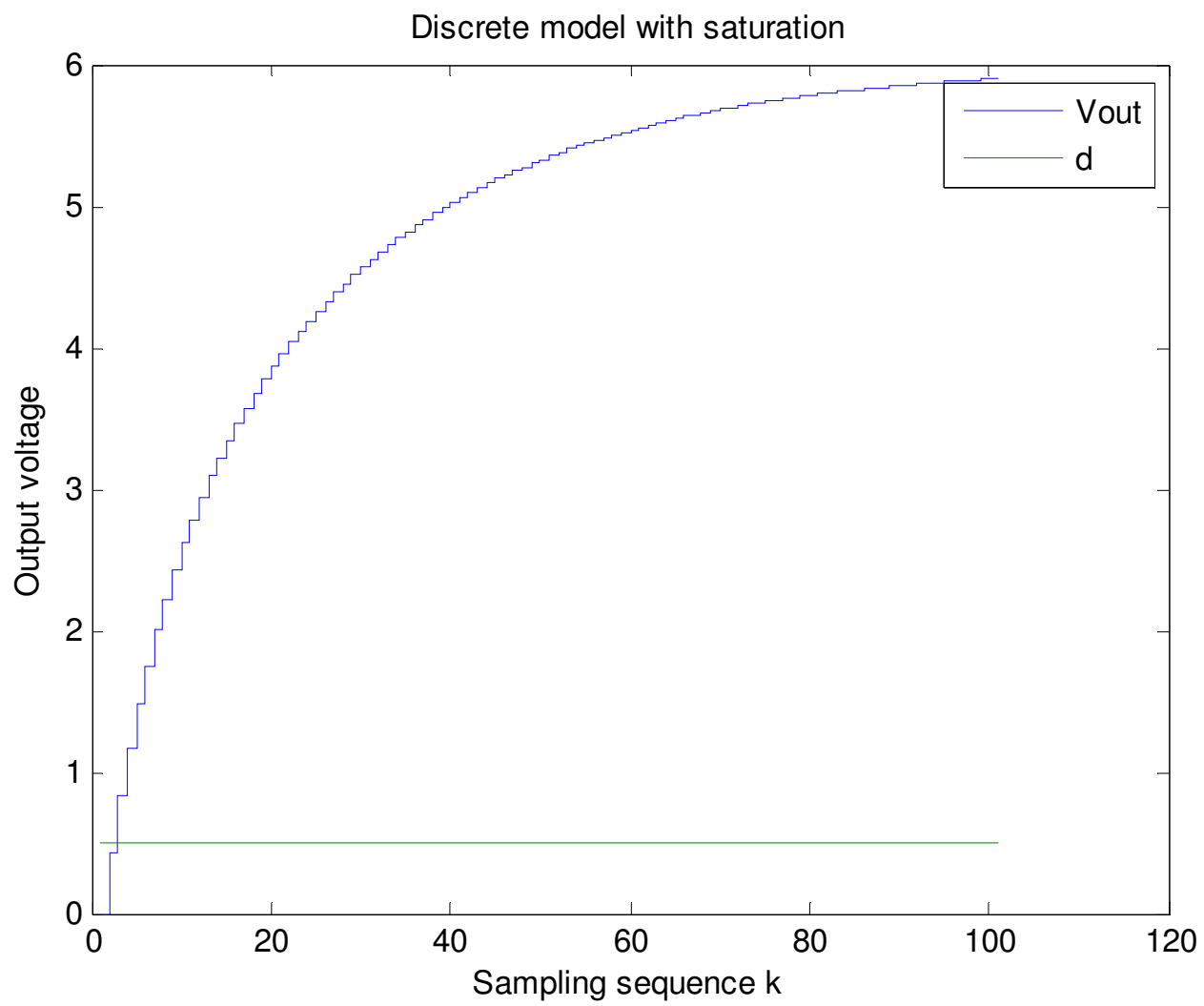

If we consider the poles at $0.21,0.24 \& 0.25$ at sampling interval $2^{\mathrm{e}-6}$, then the following are the resulted graphs

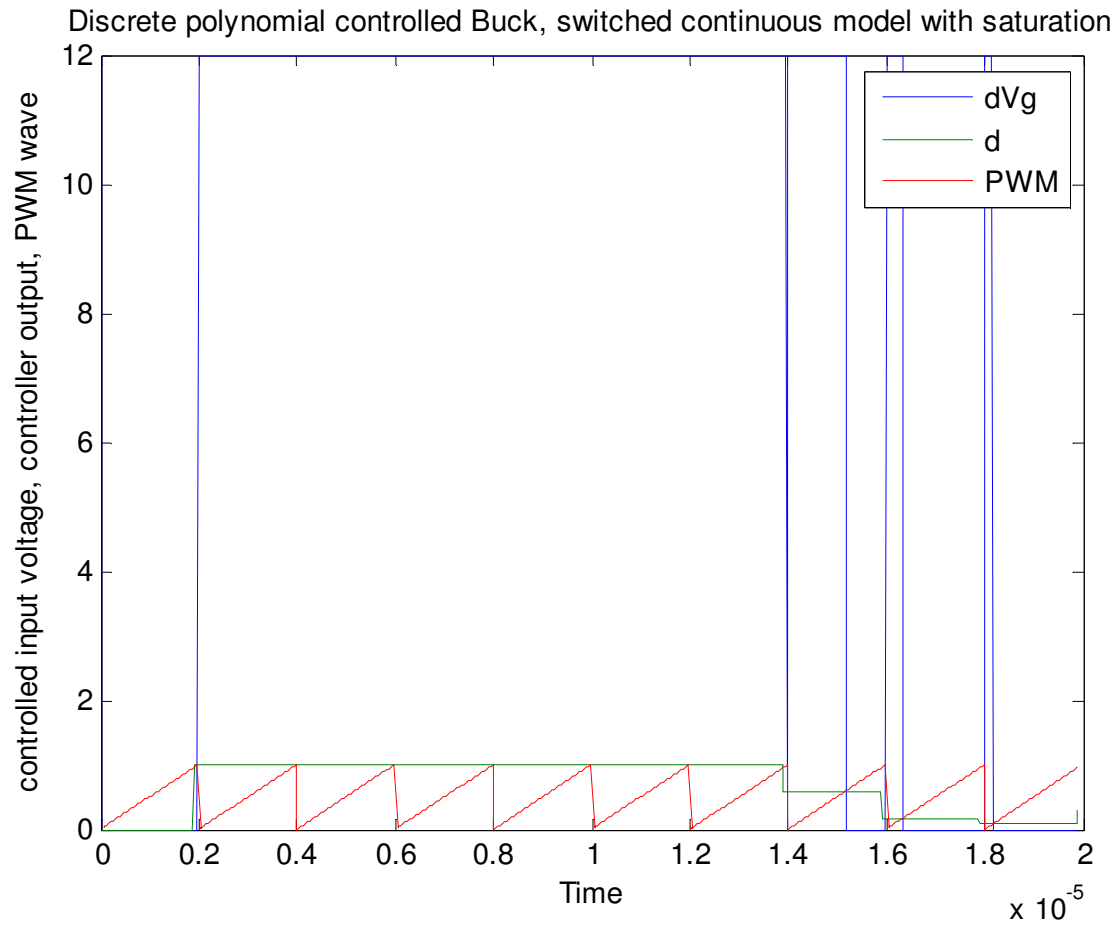


In the above figure at those poles, the $d$ value is not constant with the pwm signal, so from this simulation we can observer that at these poles the system is not stable.

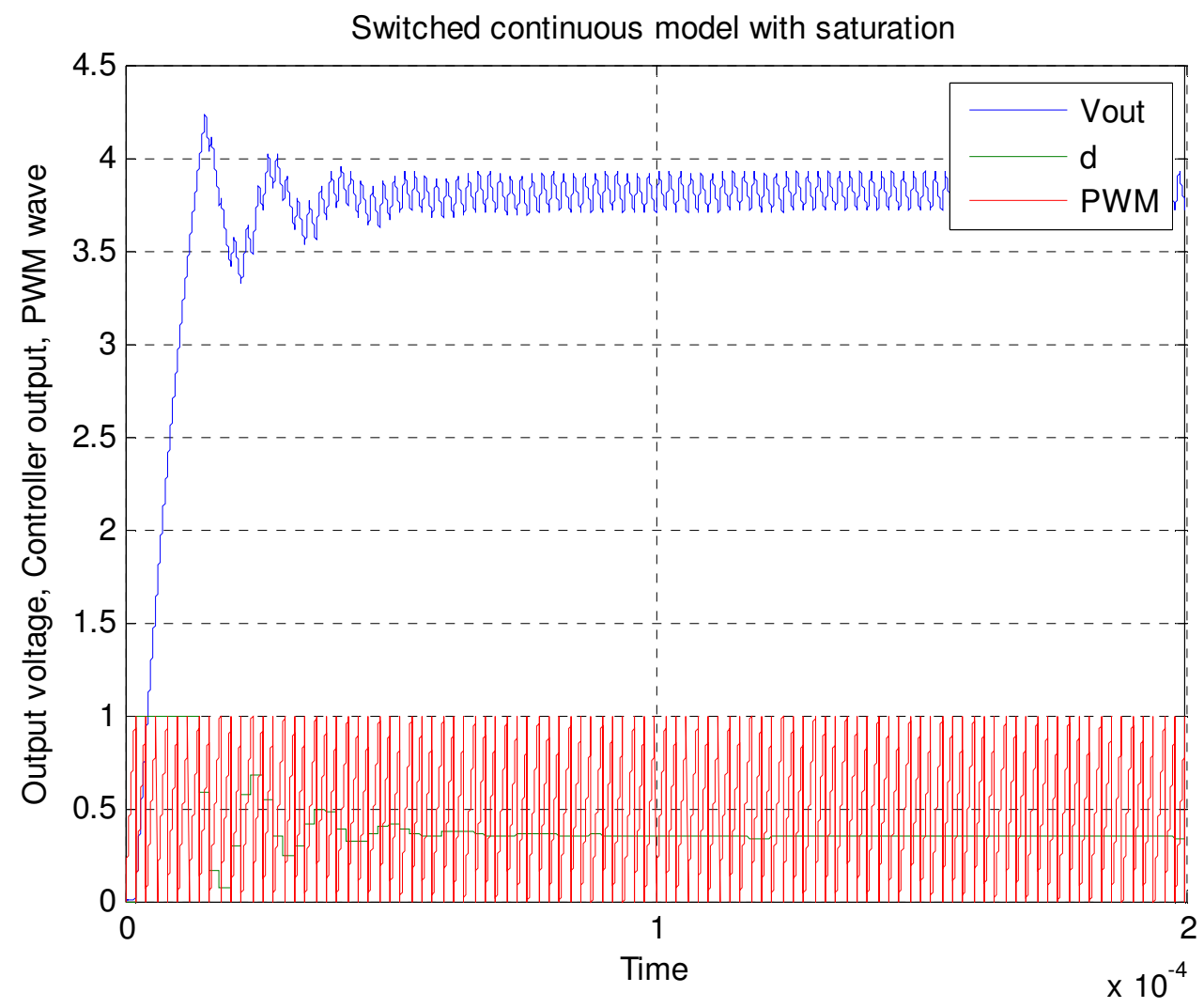

Here the reference voltage 3.9, which is very good result but the $d$ value is not constant with the pwm and $v_{\text {out }}$ is oscillating more.

The below discrete simulation the $d$ and the $v_{\text {out }}$ are shown which are good, but in the above graph the $d$ value is not constant, so the below is not valid. 


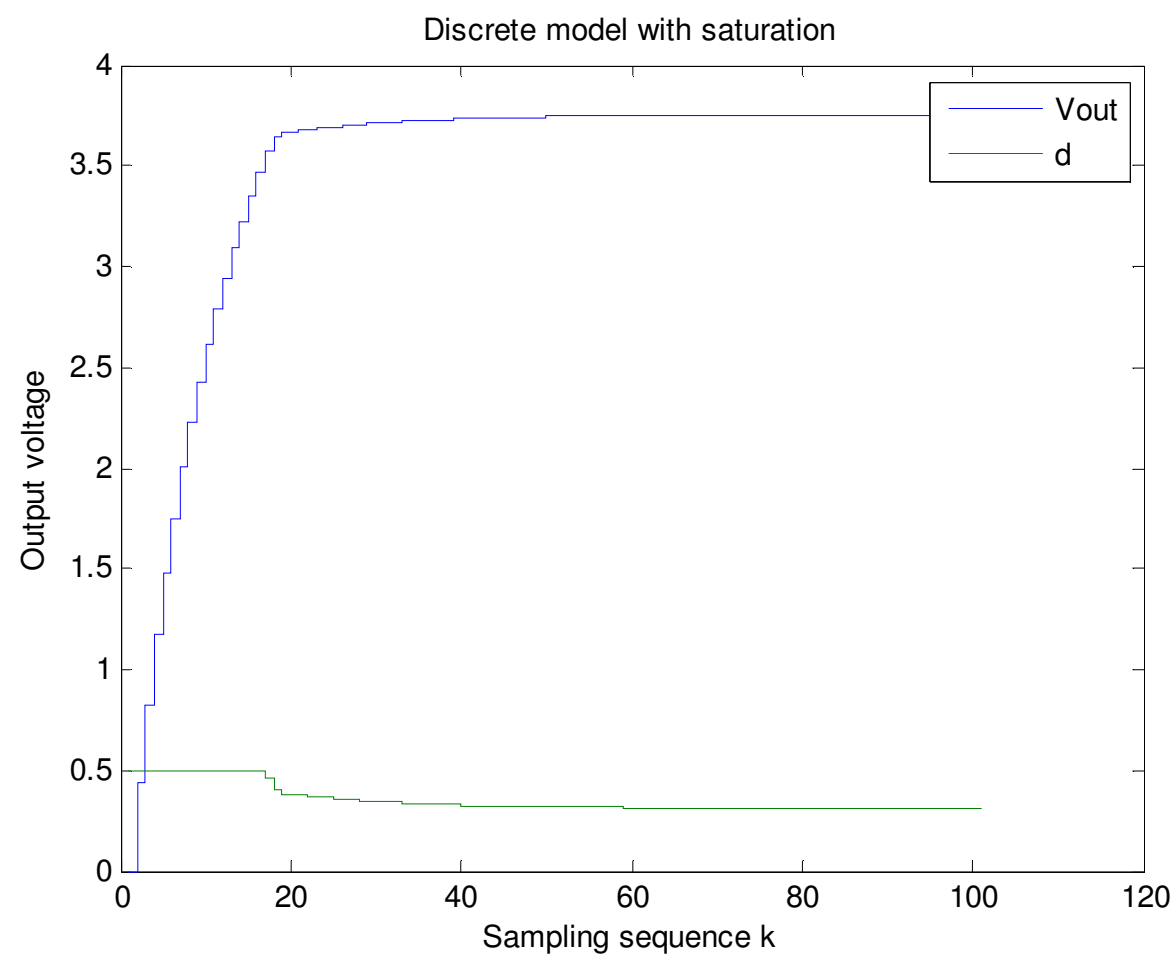




\section{Appendix 8: Matlab codes for Polynomial Pole Placement Controller:}

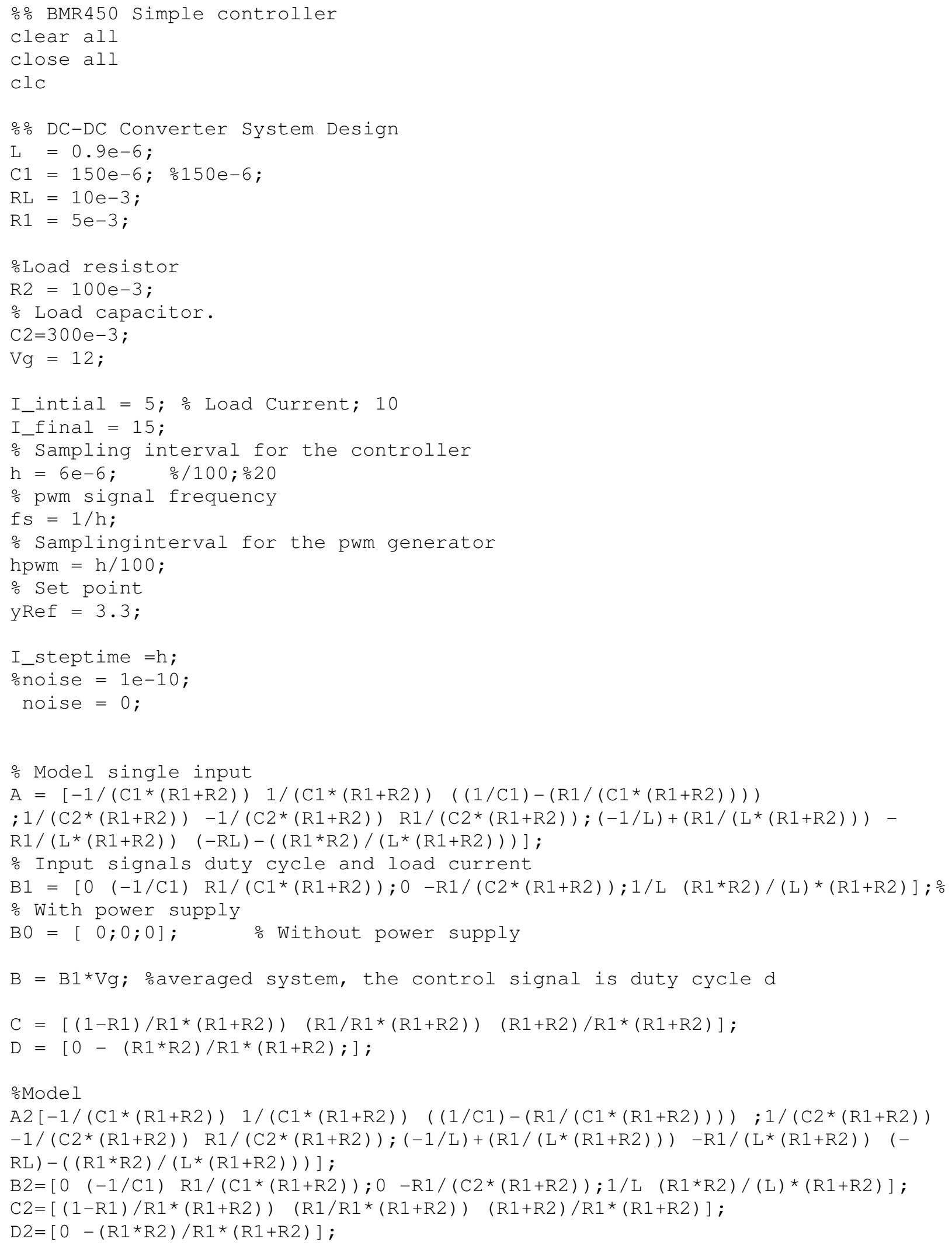




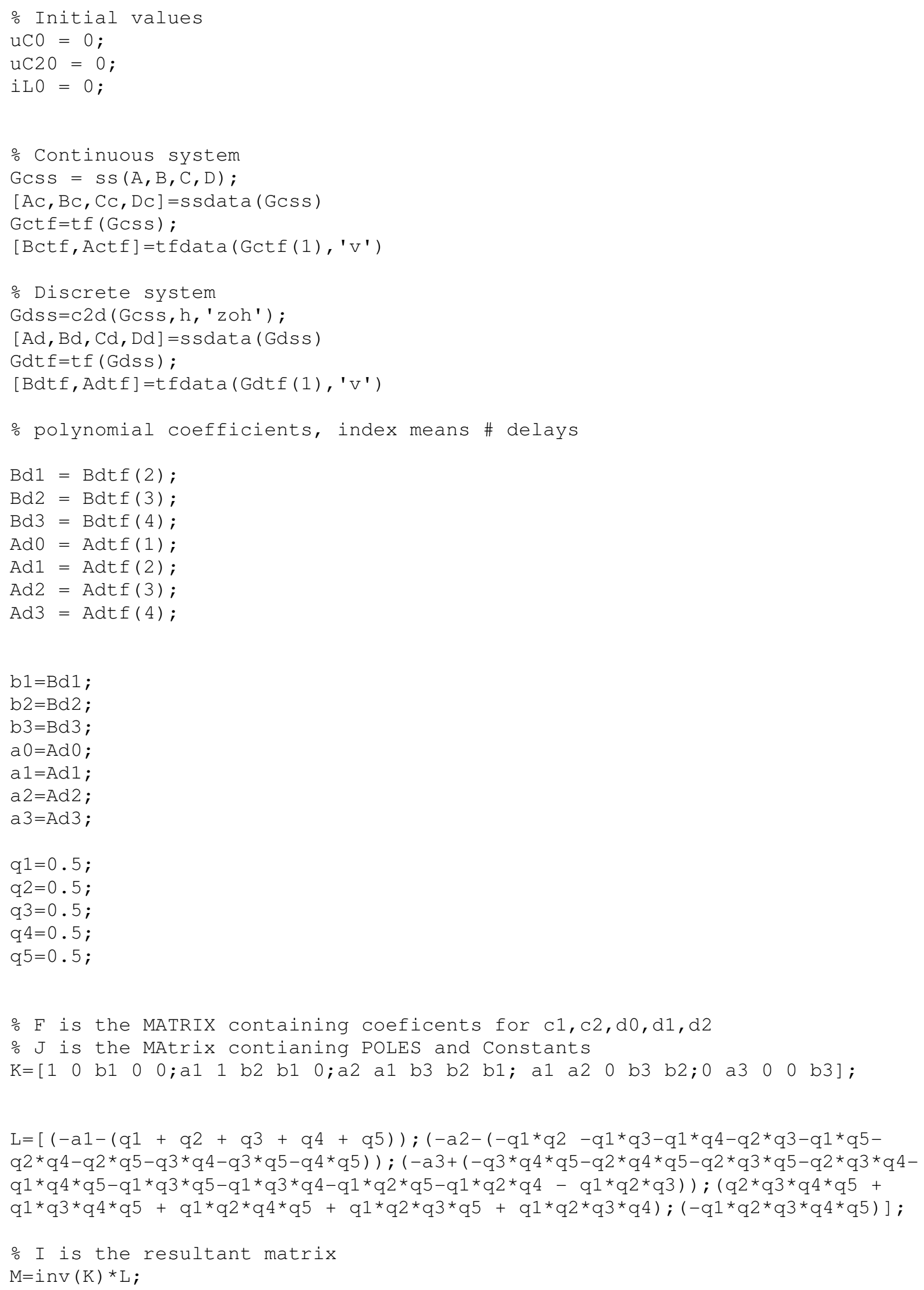




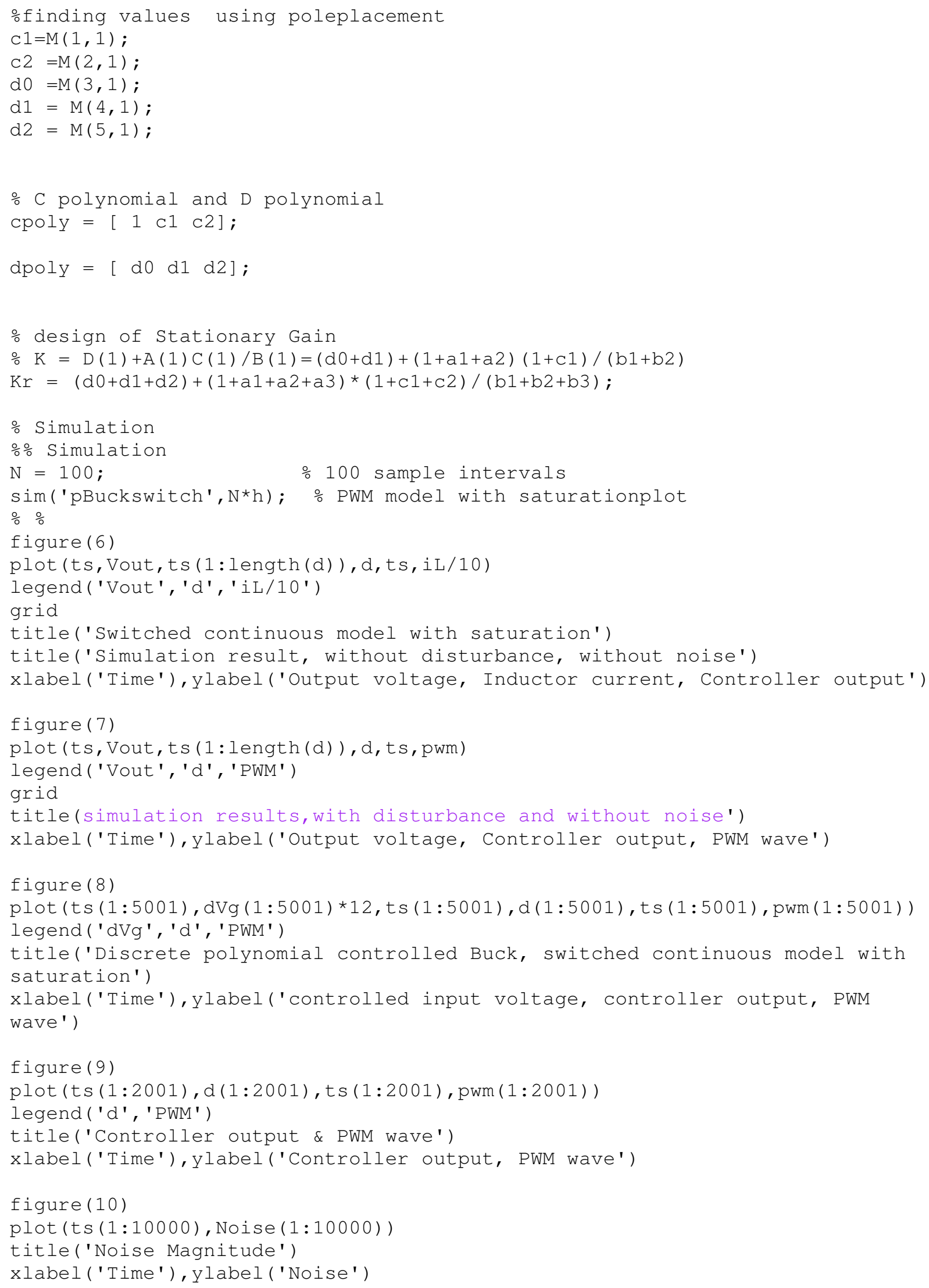


응

figure ( 11$)$

plot (ts $(1: 10000)$, Vout_N(1:10000))

legend('Without Noise')

title('Output voltage measurement without noise, without disturbance')

xlabel ('Time'), ylabel ('Measurement Noise') 


\section{Appendix 9: Matlab code for PID Controller:}

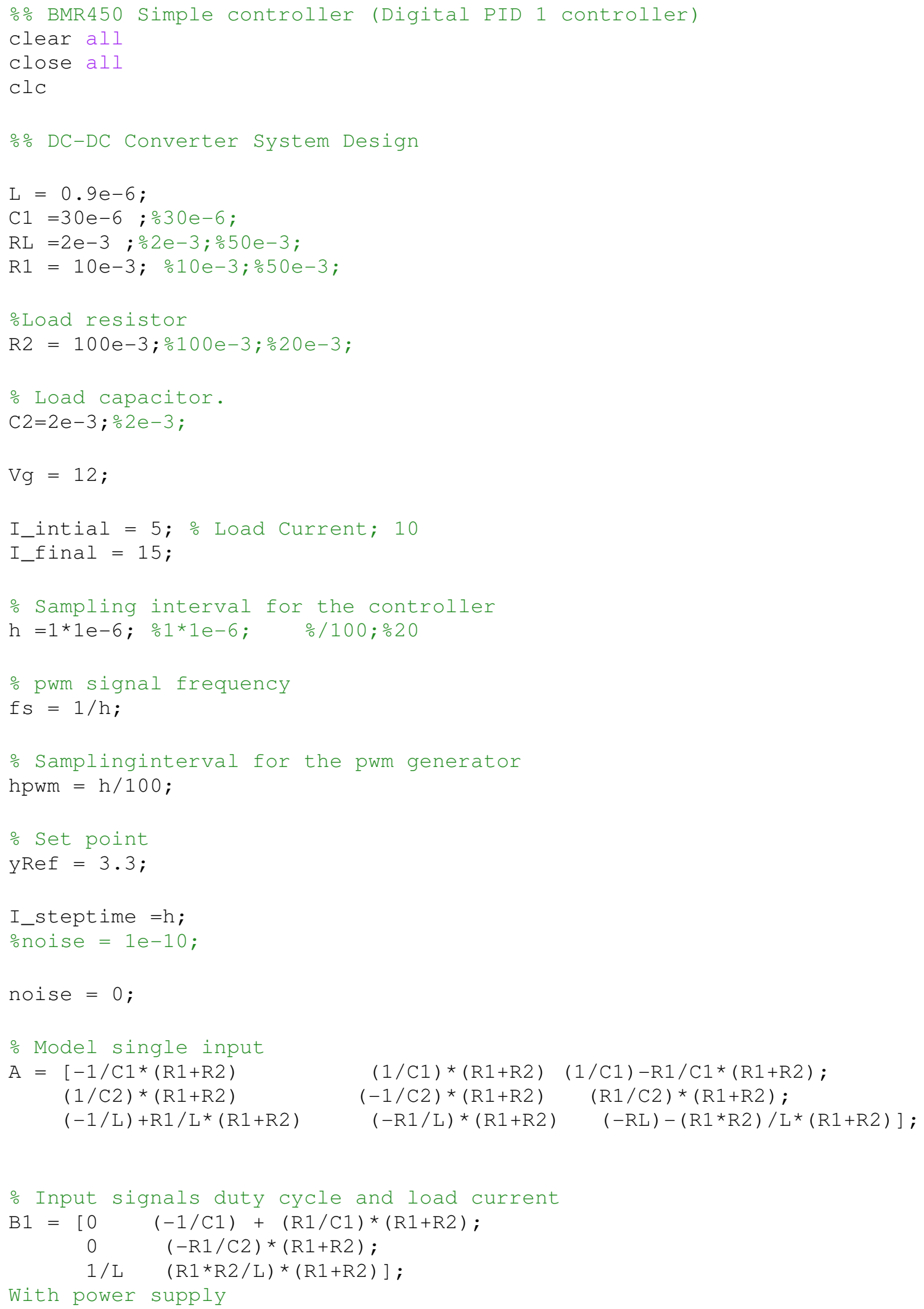




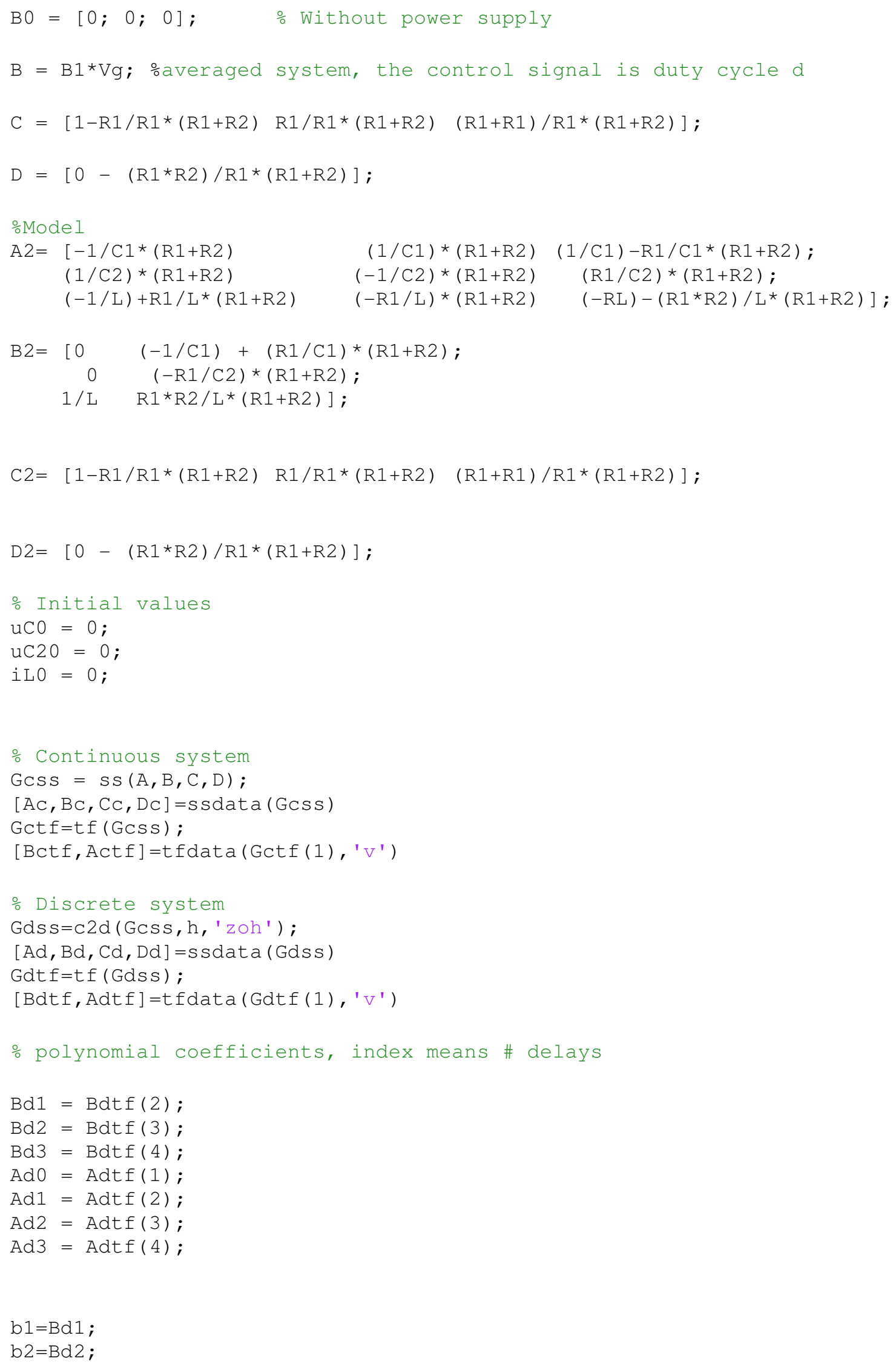




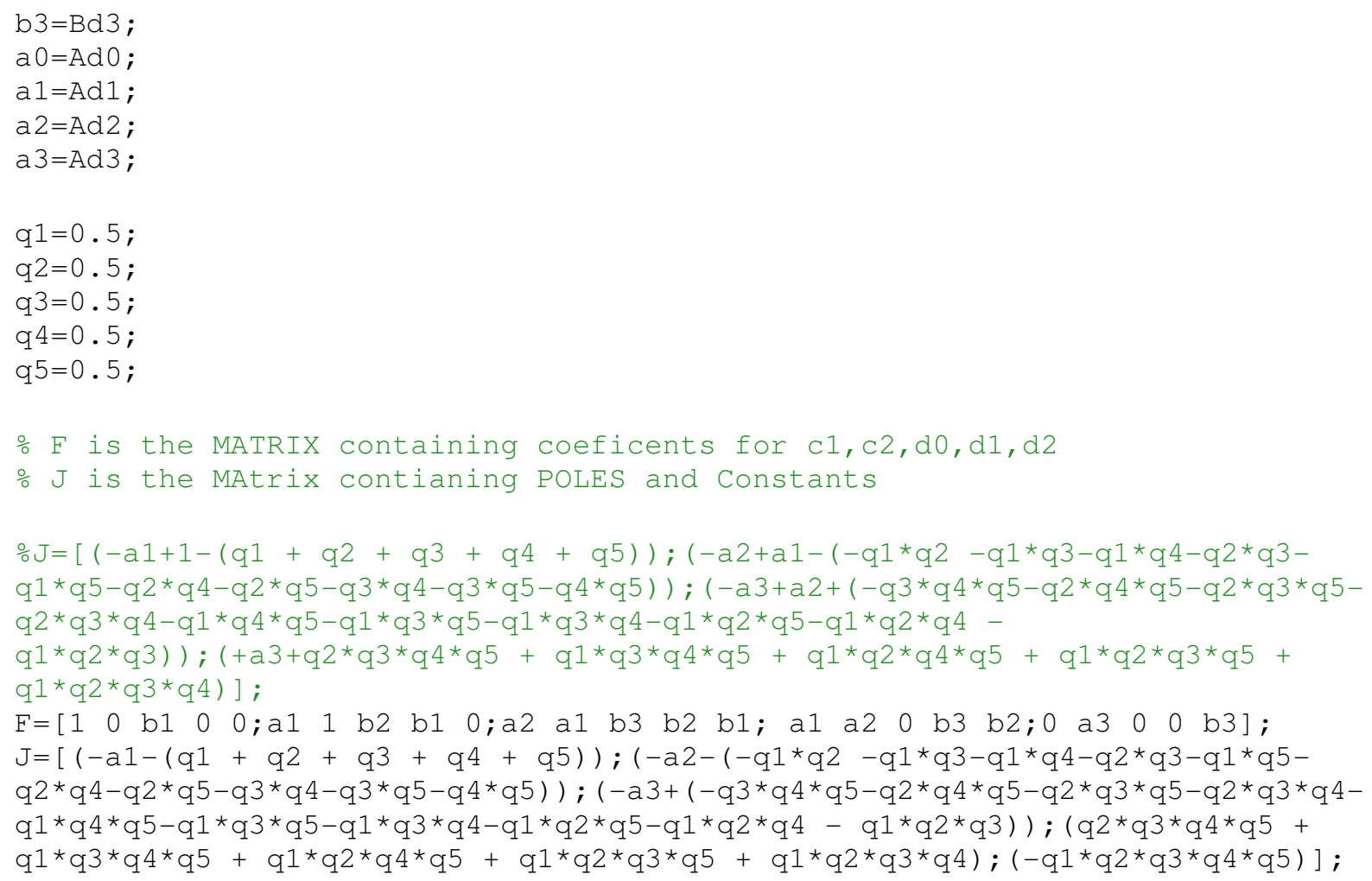




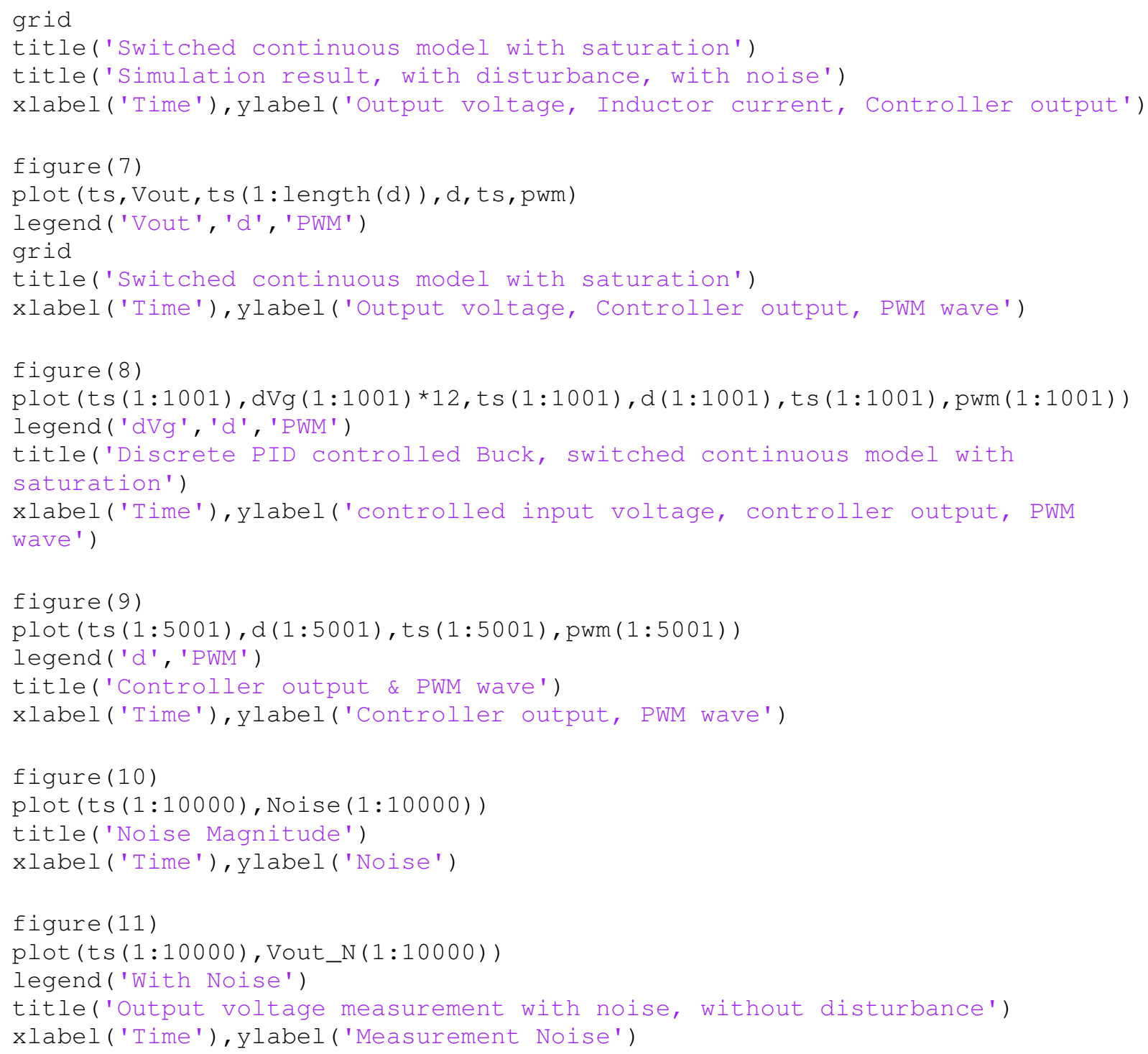




\section{Appendix 10: Matlab code for PD Controller:}

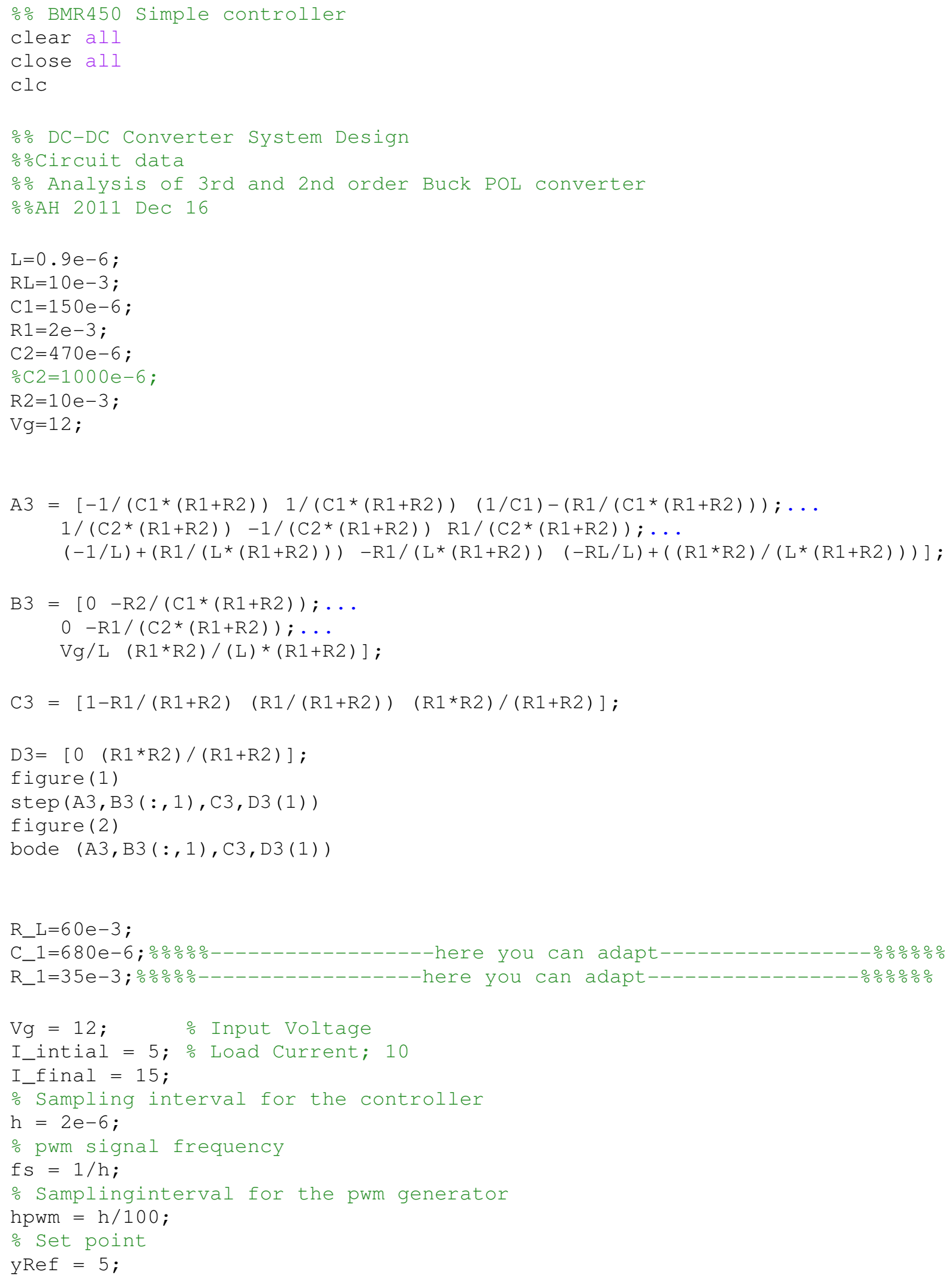




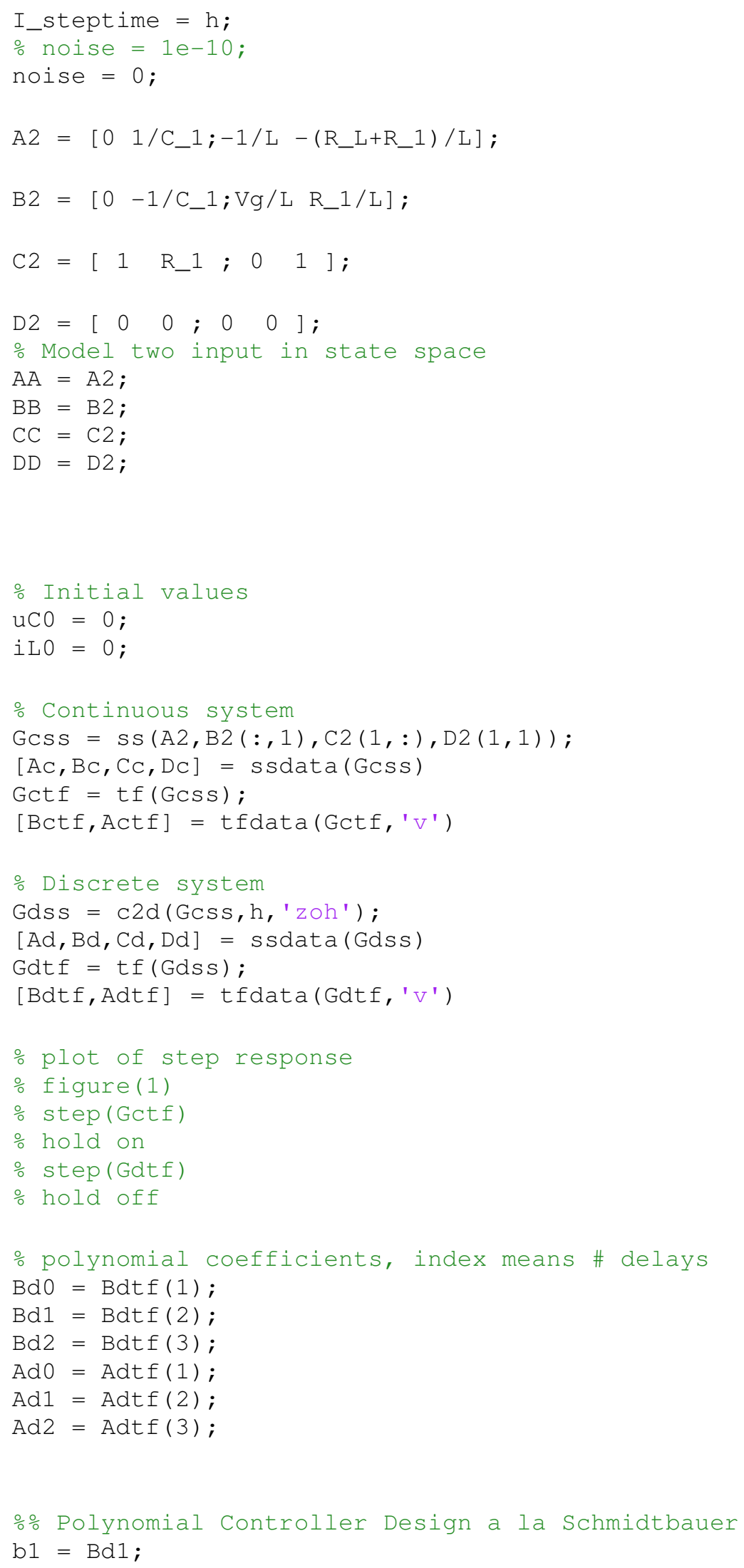




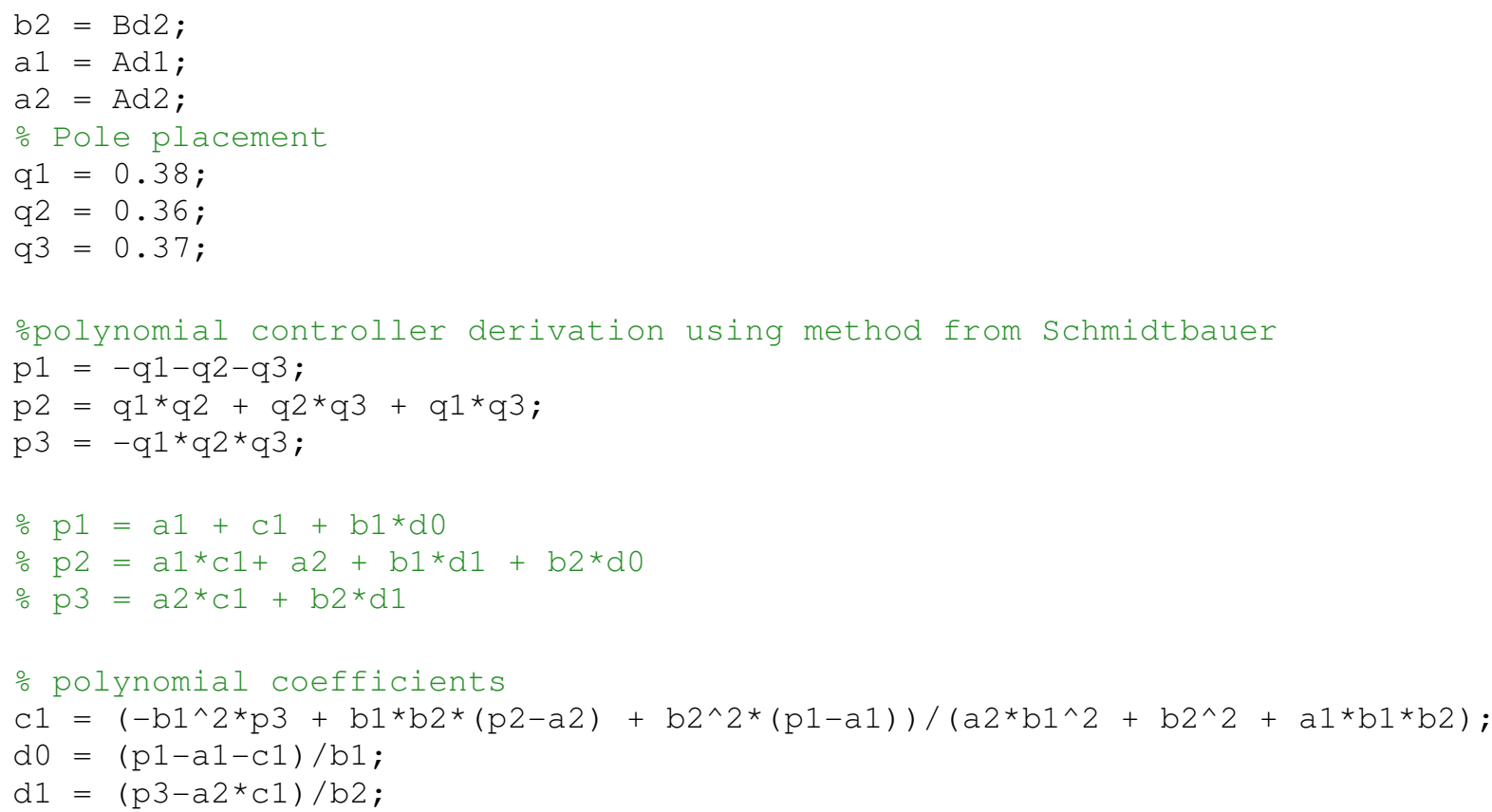

a1

a2

$\mathrm{b} 1$

$\mathrm{b} 2$

$\mathrm{c} 1$

do

d1

\% C polynomial and D polynomial

cpoly $=\left[\begin{array}{lll}1 & \mathrm{c} 1\end{array}\right]$; dpoly $=\left[\begin{array}{ll}d 0 & d\end{array}\right]$;

\% design of Stationary Gain

$\div \mathrm{Kr}=\mathrm{D}(1)+\mathrm{A}(1) \mathrm{C}(1) / \mathrm{B}(1)=(\mathrm{d} 0+\mathrm{d} 1)+(1+\mathrm{a} 1+\mathrm{a} 2)(1+\mathrm{c} 1) /(\mathrm{b} 1+\mathrm{b} 2)$

$\mathrm{Kr}=(\mathrm{d} 0+\mathrm{d} 1)+(1+\mathrm{a} 1+\mathrm{a} 2) *(1+\mathrm{c} 1) /(\mathrm{b} 1+\mathrm{b} 2)$;

\% Calculate the static gain

\% Definition of continuous system:

Csys=ss (AA, BB, CC, DD) ;

Discretizing the system:

Dsys=c2d (Csys, h, 'zoh');

- Definition of the transfer function for the controller:

Rsys=tf (dpoly, cpoly, h) ;

\% Calculation of the closed system:

Msys=feedback (Dsys, Rsys, 1, 1,-1);

\% the closed system has two inputs (d and IO) and two outputs (u0 and IL).

\% Generation of all four step responses

step (Msys)

\% calculation of all zeros, poles and static gains.

$[\mathrm{z}, \mathrm{p}, \mathrm{k}, \mathrm{Ts}]=\mathrm{zpkdata}$ (Msys)

o calculation of all transfer function

$\mathrm{Hz}=\mathrm{tf}$ (Msys) 


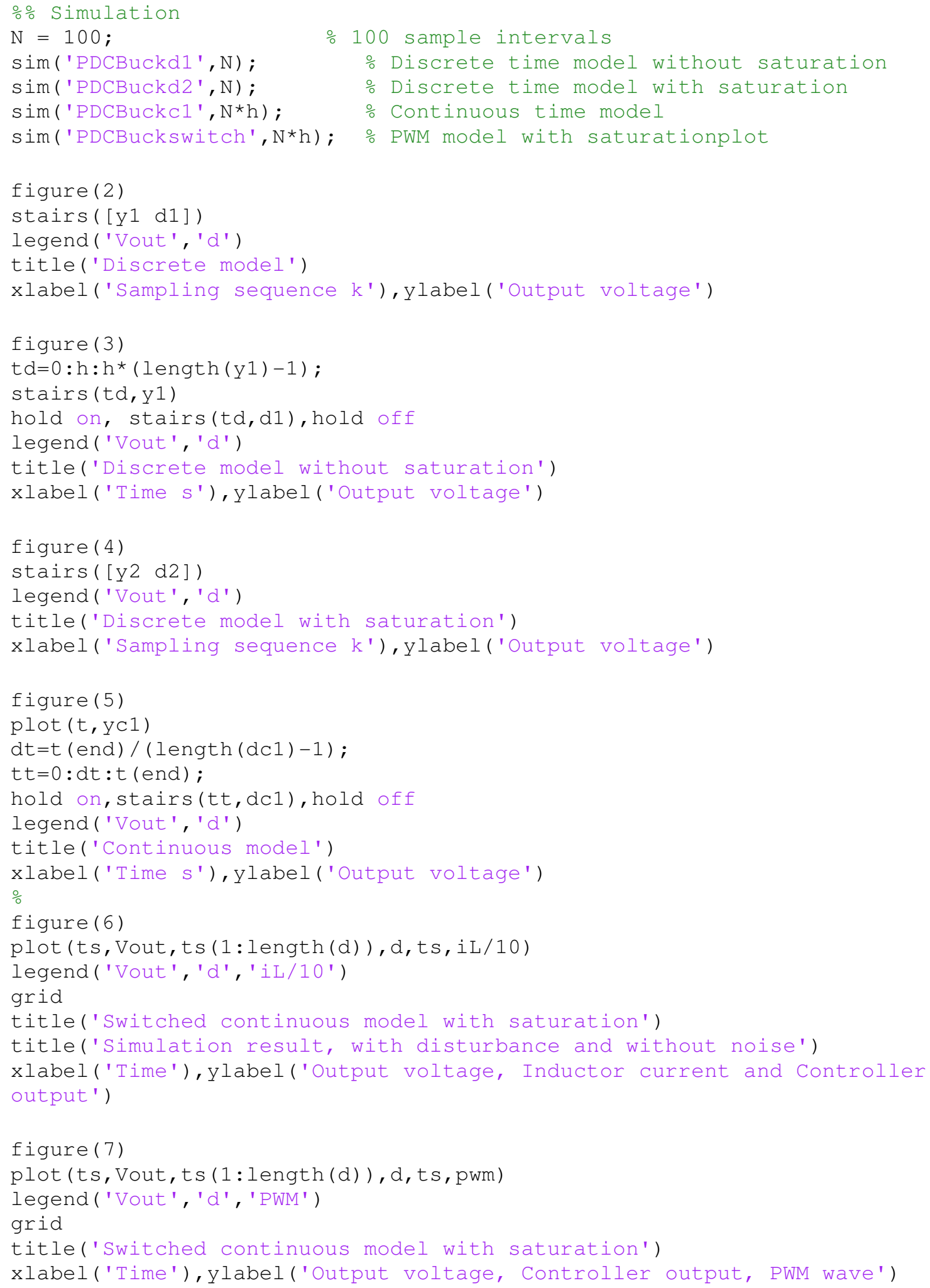




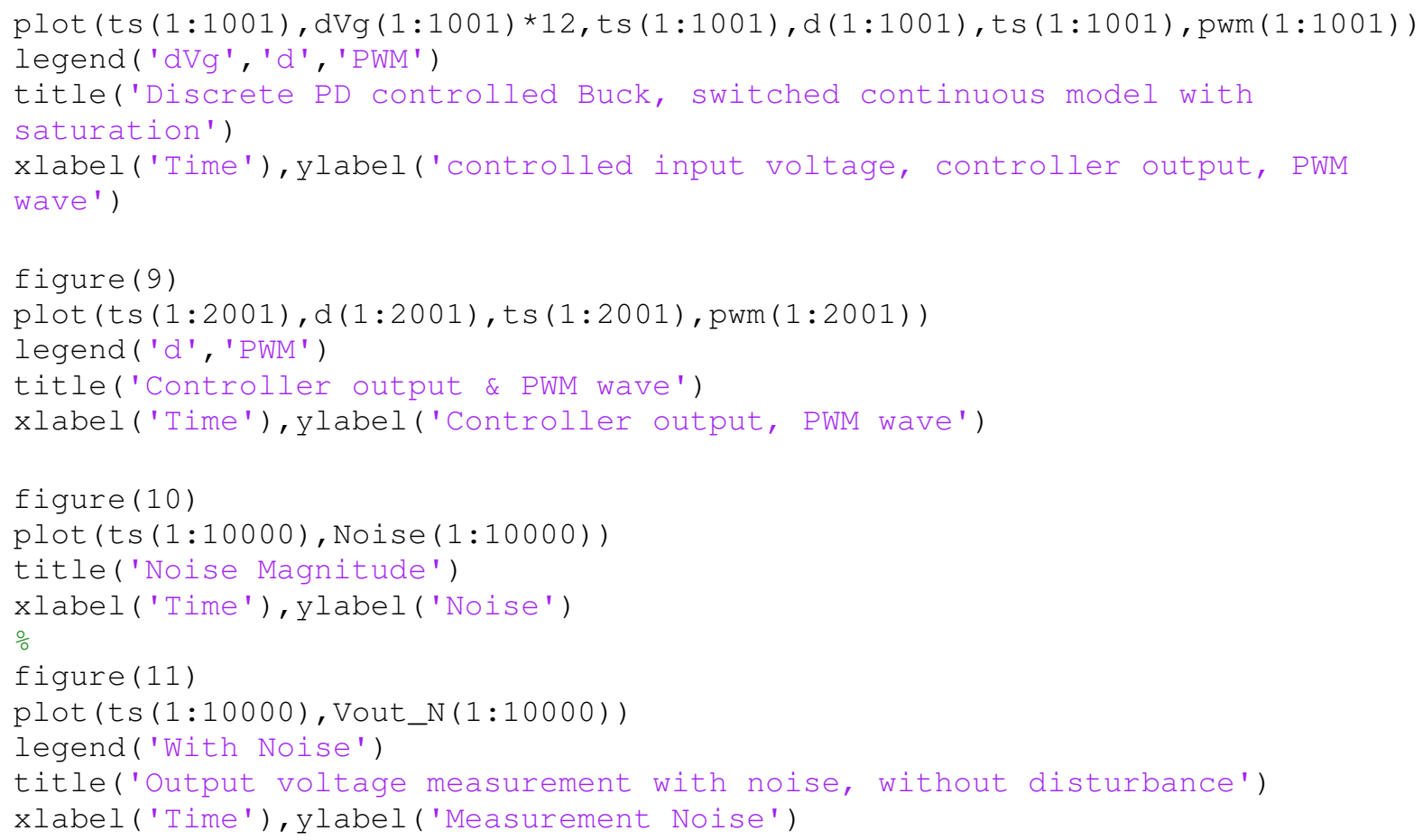

